SELECTED DRILL-STEM TEST DATA FOR

THE UPPER COLORADO RIVER BASIN

By Ralph W. Teller and Daniel T. Chafin

U.S. GEOLOGICAL SURVEY

Water-Resources Investigations Report 84-4146 


\author{
DEPARTMENT OF THE INTERIOR \\ DONALD PAUL HODEL, Secretary \\ U.S. GEOLOGICAL SURVEY
}

Dallas L. Peck, Director

For additional information write to:

District Chief

U.S. Geological Survey, Box 25046, Mail Stop 415

Denver Federal Center

Denver, C0 80225
Copies of this report can be purchased from:

U.S. Geological Survey Books and Open-File Reports Building 41, Box 25425

Denver Federal Center

Denver, C0 80225

[Telephone (303) 236-7476] 
Abstract-1-0 1

Introduction-1.-1 1

Location of study area-1

Location of drill-stem test sites-c 3

Drill-stem tests---_en 3

Drill-stem test data-10

Selected references-- 109

\section{ILLUSTRATIONS}

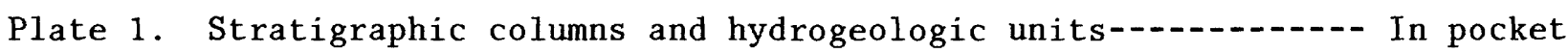

Page

Figure 1. Map showing location of study area- 2

2. Diagram showing system of identifying drill-site locations---- 4

3. Principal tectonic features of the Upper Colorado River Basin-... 5

4. Graph showing relation between viscosity and water temperature--.- 6

5-15. Maps showing locations of drill-stem test data for formations in the following hydrogeologic units:

5. All hydrogeologic units-14

6. Upper Tertiary discontinuous aquifers-_. 15

7. Middle Tertiary aquifers, partly drained aquifers, and confining layers-_... 17

8. Lower Tertiary aquifers and confining layers---_--- 19

9. Basal Tertiary aquifers-_... 36

10. Upper Mesozoic confining layers and aquifers-_.... 41

11. Middle Mesozoic aquifers-_ 72

12. Lower Mesozoic confining layers-_- 79

13. Upper Paleozoic aquifers and confining layers---.-- 82

14. Middle Paleozoic aquifers-_... 99

15. Lower Paleozoic confining layers and aquifers------ 107 


\section{CONTENTS}

\section{TABLES}

Page

Table 1. Geologic codes and formation names---11

2-11. Drill-stem test data for:

2. Upper Tertiary discontinuous aquifers-_-_- 16

3. Middle Tertiary aquifers, partly drained aquifers, and confining layers--_- 18

4. Lower Tertiary aquifers and confining layers--------- 20

5. Basal Tertiary aquifers---- 37

6. Upper Mesozoic confining layers and aquifers------- 42

7. Middle Mesozoic aquifers--- 73

8. Lower Mesozoic confining layers---_--- 80

9. Upper Paleozoic aquifers and confining layers--------- 83

10. Middle Paleozoic aquifers---_-_-_-_-_-_-_-_-_---- 100

11. Lower Paleozoic confining layers and aquifers--------- 108

\section{METRIC CONVERSIONS}

The inch-pound units used in this report may be converted to SI (International System of Units) by use of the following conversion factors:

Multiply inch-pound unit

\section{barrel}

foot ( $f t$ )

foot per day $(\mathrm{ft} / \mathrm{d})$

mile (mi)

millidarcy

square mile $\left(\mathrm{mi}^{2}\right)$

pound per square inch $\left(1 b /\right.$ in $\left.^{2}\right)$

Degree Fahrenheit $\left({ }^{\circ} \mathrm{F}\right)$
By

0.159

0.3048

0.3048

1.609

0.000987

2.590

6.895

${ }^{\circ} \mathrm{C}=5 / 9\left({ }^{\circ} \mathrm{F}-32\right)$
To obtain SI unit

cubic meter

meter

meter per day

kilometer

square micrometer

square kilometer

kilopascal

degree Celsius $\left({ }^{\circ} \mathrm{C}\right)$ 


\title{
SELECTED DRILL-STEM TEST DATA FOR THE UPPER COLORADO RIVER BASIN
}

\author{
By Ralph W. Teller and Daniel T. Chafin
}

\section{ABSTRACT}

Drill-stem test data collected during petroleum exploration can be analyzed to provide information on formation head and permeability that is needed for regional hydrologic investigations. Approximately 2,000 drill-stem tests were analyzed as part of a ground-water study of a part of the Upper Colorado River Basin. Analyses of these drill-stem tests provided values for undisturbed formation head and, in some cases, permeabilities that were converted to intrinsic permeabilities and hydraulic conductivities. Results of these analyses are presented in tabular form. Information collected on the hydrologic characteristics of the formations tested will be used for flowsystem analysis, including simulation modeling.

\section{INTRODUCTION}

Regional studies of ground-water resources and aquifer systems require analysis of the hydrologic properties of the system, including aquifers and confining layers. In some areas, such information may be available from drill-stem tests made during the course of petroleum exploration. Drill-stem data were purchased from Roger Hoeger (written commun., 1980) for the analyses presented in this report. As part of the Upper Colorado River Basin regional aquifer system analysis, approximately 2,000 drill-stem tests were analyzed. This report summarizes these analyses and provides permeability data that can be used by hydrologists and other earth scientists. Data from the upper part of the San Juan drainage basin will be included in other studies and are not presented in this report.

\section{LOCATION OF STUDY AREA}

The Upper Colorado River Basin (fig. 1) comprises the drainage basin of the Colorado River above Lees Ferry, Ariz. The upper basin is on the west side of the Continental Divide and includes parts of Arizona, Colorado, New Mexico, Utah, and Wyoming. It encompasses an area of $113,500 \mathrm{mi}^{2}$ and extends from latitude $35^{\circ} 34^{\prime} \mathrm{N}$. to $43^{\circ} 27^{\prime} \mathrm{N}$., a distance of about $550 \mathrm{mi}$, and from longitude $105^{\circ} 38^{\prime} \mathrm{W}$. to $112^{\circ} 19^{\prime} \mathrm{W}$., a distance of about $350 \mathrm{mi}$. 


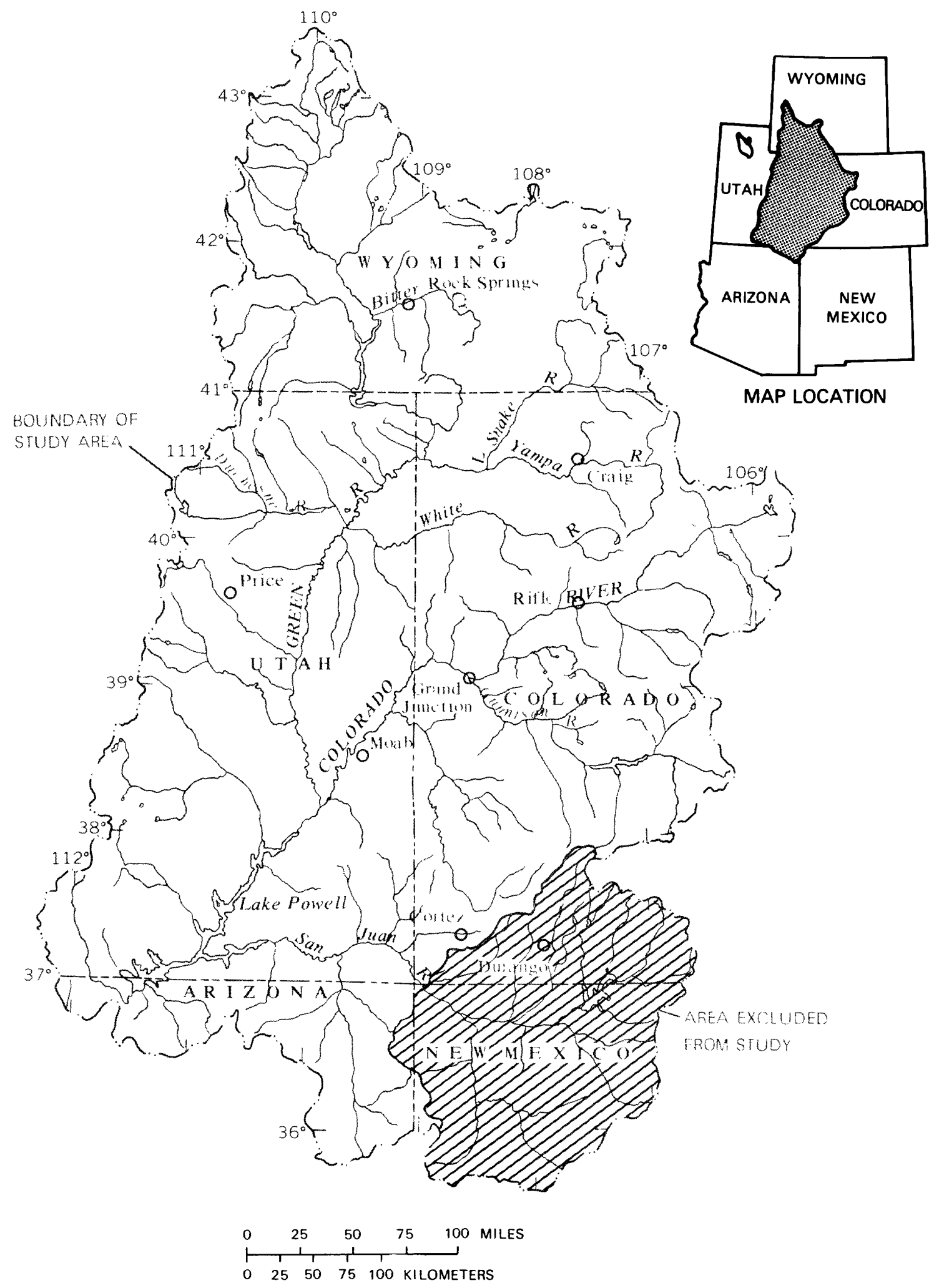

Figure 1.--Location of study area. 
The Upper Colorado River Basin is characterized by rugged mountains, high plateaus, broad expanses of desert, and narrow valleys. It is bounded on the east and north by mountains forming the Continental Divide and on the west by the Wasatch Mountains and the Wyoming Range. It is bounded on the south by plateaus and small mountain ranges that separate the San Juan River drainage from the Little Colorado River drainage. Discharge from the Upper Colorado River Basin to the lower basin is at Lees Ferry in northern Arizona.

The main valleys of the Upper Colorado River Basin have been cut by the Colorado River and its principal tributaries, the Gunnison, Green, and San Juan Rivers. Altitudes range from $3,100 \mathrm{ft}$ at Lees Ferry to more than $14,000 \mathrm{ft}$ on many of the mountain peaks.

\section{LOCATION OF DRILL-STEM TEST SITES}

Drill-stem tests included in this report are listed by the drill-site location, using the U.S. Bureau of Land Management System of Land Subdivision. Site locations are identified by their township, range, and section numbers, as illustrated by example in figure 2 .

\section{DRILL-STEM TESTS}

Dri11-stem test data are presented in the Drill-Stem Test Data section in tables 1-11; these data are grouped according to the formations within the hydrogeologic units shown on plate 1 (in the pocket in the back cover). These units are classified on the basis of depositional environment and lithology. Geologic formations within which the tests were conducted are identified by a formation code in table 1 . Stratigraphic columns on plate 1 also indicate the geologic age of many of the formations tested (the complex stratigraphy prevents all members from being included in the columns) and their relation to principal tectonic features (fig. 3). Some drill sites may have data from tests done at several intervals or depths in the same drill hole. These data may reflect differences in physical conditions, for example fluid temperature and viscosity, at the given depth or interval tested. Permeability data were corrected for variations in viscosity; curves depicting the relationship between viscosity and temperature for this correction are shown in figure 4. Figures 3 and 4 follow this discussion immediately. Maps (figs. 5 through 15) are placed in the Dri11-Stem Test Data section at the back of the report, preceding each relevant table, to indicate the general locations of test sites and areal distribution of test data within hydrogeologic units. 


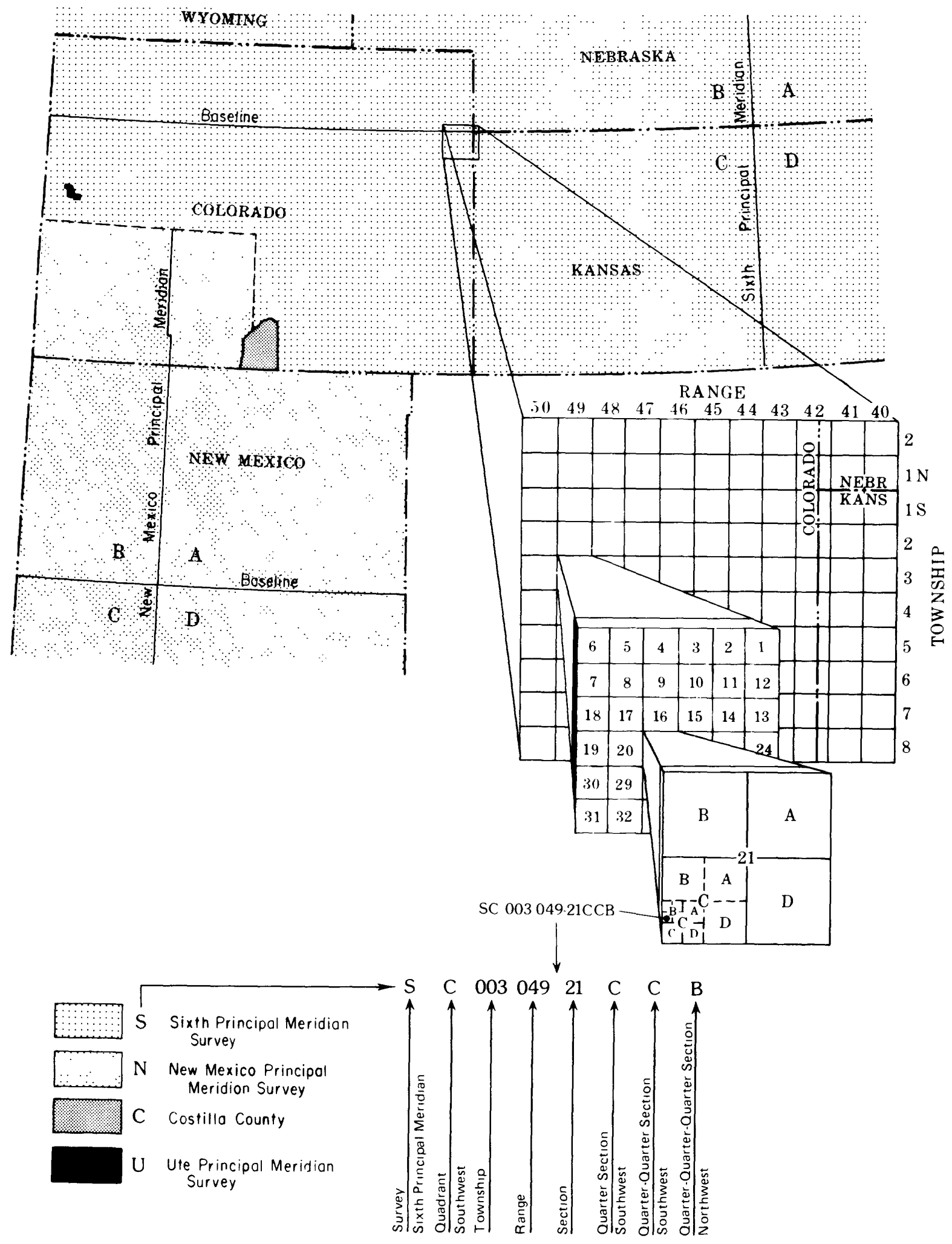

Figure 2.--System of identifying drill-site locations. 


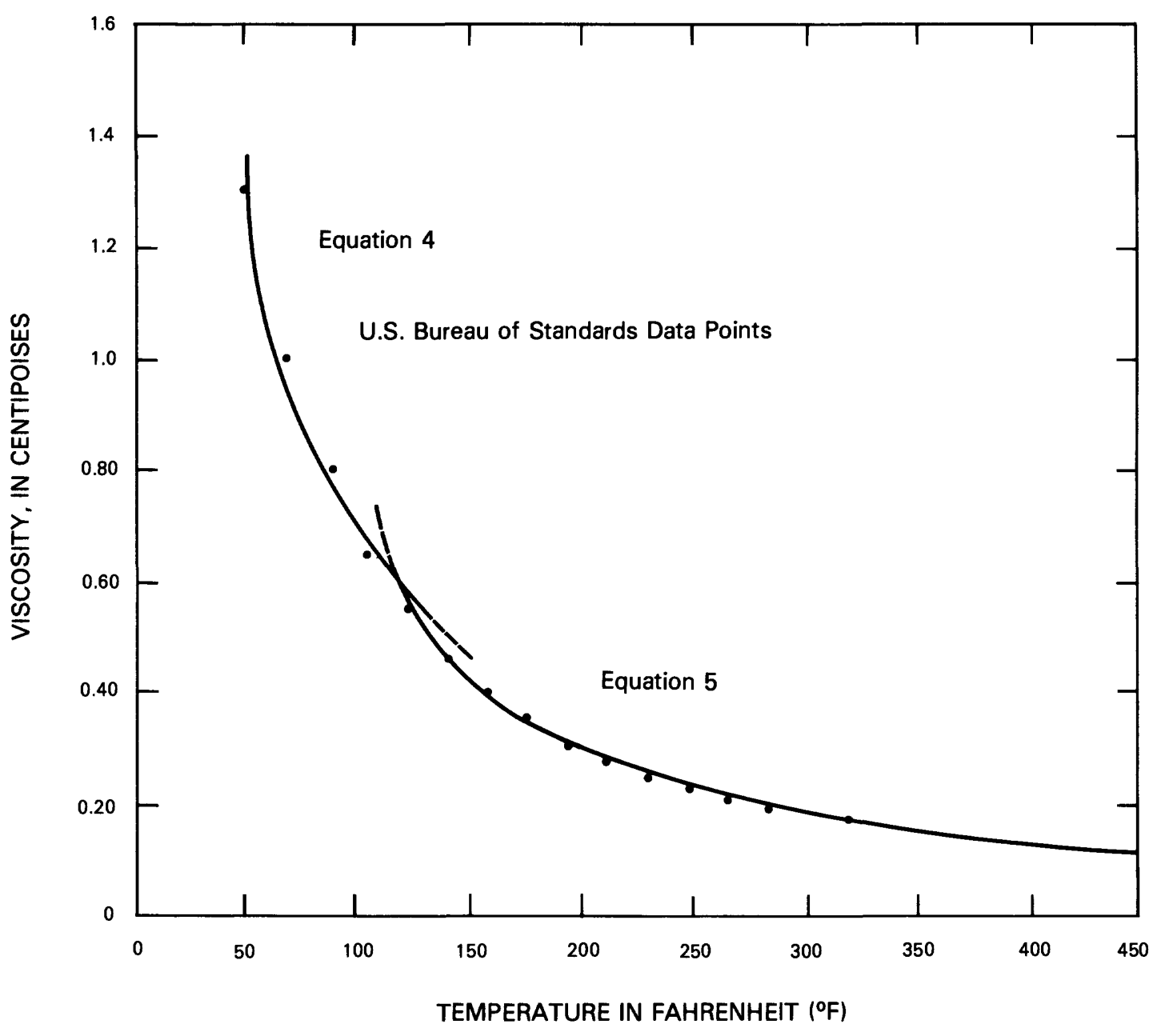

Figure 4. - Relation between viscosity and water temperature. 
A drill-stem test is much like a bail test or a short-term aquifer recovery test. A volume of fluid is removed from the well bore during a known period of time; then, the fluid head is allowed to recover. Analysis of the recovery data provides information on the pressure and permeability of the formation in the interval tested. Values obtained from this analysis may be influenced by localized oil field production activity. Some high shut-in heads may indicate that the formation is abnormally overpressured.

In a typical drill-stem test, the stratigraphic interval of interest in the hole is isolated from the rest of the hole by the use of packers attached to the bottom of the drill stem. After the drill stem has been lowered into the well bore, and the packers have been expanded to seal off and isolate the interval to be tested, a valve is opened to allow fluid to flow from the formation into the drill stem, under the influence of the head difference between the formation and the atmosphere in the drill stem. The valve is then closed, or shut in, and the head recovery of the formation is monitored by a pressure sensor open to the well bore in the tested interval.

In addition to the pressure measurements that provided information on static formation head and permeability, fluid-temperature data also were collected during the tests. In most cases, fluid recovered from the drill stem can be analyzed for chemical composition.

Drill-stem tests contained in this report were selected on the basis of meeting certain quality criteria, including:

1. Pressure curves that did not exhibit any effects from plugging;

2. No pressure loss from packer slippage or malfunction;

3. Sufficient period of monitoring pressure recovery following shut-in of the flow valve; and

4. No malfunctions of the pressure gage.

Tests that met these criteria were analyzed by means of the Horner graphical method (Bredehoeft, 1965). Pressure was plotted as the ordinate on an arithmetic scale, versus $\frac{t+\theta}{\theta}$ as the abscissa on a logarithmic scale, where $t=$ duration of flow preceding shut-in, and $\theta=$ time since shut-in of flow valve.

The resulting plot should be linear, if the test behaves ideally. If a straight-line plot was obtained, the best-fit line through the data was

extrapolated to $\log \frac{t+\theta}{\theta}=1$, which was equivalent to an infinite time since shut-in. The pressure at this time was an estimate of the pressure to which the formation will recover. This intercept pressure was taken as the original formation pressure $\left(P_{f}\right)$ at the gage altitude. 
Undisturbed, or shut-in head, presented as the altitude of the potentiometric surface in terms of fresh-water equivalent, was calculated by means of the equation:

$$
H=A-D+2.33 P_{f}
$$

where $H=$ shut-in head $(\mathrm{ft})$,

$$
\begin{aligned}
A= & \text { land-surface altitude of bore hole }(\mathrm{ft}), \\
D= & \text { depth below land surface at which pressure was } \\
& \text { measured }(\mathrm{ft}), \text { and } \\
P_{f}= & \text { extrapolated or stabilized formation pressure }\left(1 \mathrm{~b} / \mathrm{in}^{2}\right) . \text { The }
\end{aligned}
$$

constant 2.33 converts pressure to equivalent fresh-water head. In some cases, it was found that shut-in pressures stabilized and that the Horner graphical extrapolation method was not necessary. In these cases, shut-in head was calculated directly by using the stabilized pressure in the above equation.

Several forms of permeability data also are included in tables 2-11 in the Drill-stem Test Data section of this report. Permeabilities in millidarcies per centipoise resulted from the standard drill-stem test analysis. Intrinsic permeabilities were calculated to indicate formation characteristics; hydraulic conductivities were calculated to indicate the formation and fluid characteristics for ground-water flow analysis.

If the fluid recovered was mostly water (mud or oil, less than 15 percent by volume) and a linear Horner plot was obtained, permeabilities were calculated by the equation:

$$
\frac{k h}{\mu}=\frac{162.6}{m} Q,
$$

where $k=$ intrinsic permeability (millidarcy),

$h=$ thickness of test interval ( $f t$ ),

$\mu=$ dynamic viscosity of fluid (centipoise),

$Q=$ fluid recovery in barrels per day, and

$m=$ change in gage pressure over one log cycle in Horner plot. $Q$ is calculated by multiplying the number of feet of fluid recovery by the capacity (in barrels/ft) of drill collars and pipes and multiplying by

$$
\frac{1,440 \text { minutes per day. }}{t \text { (minutes) }}
$$


Permeability values in millidarcies per centipoise were converted to intrinsic permeability values in millidarcies by multiplying by dynamic fluid viscosity. Intrinsic permeability was then converted to hydraulic conductivity, $K$, in feet per day at $60^{\circ} \mathrm{F}$, by the following equation:

$$
k \times \frac{18.2 \times 10^{-3}}{7.48}=K .
$$

Conversion from millidarcies per centipoise to intrinsic permeability requires a value for dynamic viscosity of the fluid. This value is a function of the temperature of the fluid. To generate the viscosity at an indicated fluid temperature, equations were used to approximate the viscosity value of water for temperatures over the range of the data. For the range:

$$
\begin{aligned}
& 50^{\circ} \mathrm{F} \text { to } 120^{\circ} \mathrm{F}, \mu=1.9299-0.81807 \log \left(\frac{5 \times \mathrm{T}\left({ }^{\circ} \mathrm{F}\right)}{9}-22.78\right) . \\
& 120^{\circ} \mathrm{F} \text { to } 425^{\circ} \mathrm{F}, \mu=0.9353-0.3670 \log \left(\frac{5 \times \mathrm{T}\left({ }^{\circ} \mathrm{F}\right)}{9}-57.80\right) .
\end{aligned}
$$

Curves for the two equations were compared with Bureau of Standards tabulated values of viscosity as a function of temperature (fig. 4).

No viscosity correction was made for dissolved-solids concentration in the fluid. Users having sufficient water-quality information available may wish to consult U.S. Geological Survey Open-File Report 82-447 (Weiss, 1982) for dissolved-solids correction techniques. 
DRILL-STEM TEST DATA 
Table 1.--Geologic codes and formation names

\begin{tabular}{|c|c|}
\hline CODE & FORMATION NAME \\
\hline OOOIGNS & IGNEOUS ROCKS \\
\hline 120TRTR ${ }^{1}$ & TERTIARY SYSTEM \\
\hline 124DGCK & DOUGLAS CREEK MEMBER (GREEN RIVER FORMATION) \\
\hline $124 \mathrm{EOCN}$ & EOCENE SERIES \\
\hline $124 \mathrm{GDGC}$ & GARDEN GULCH MEMBER (GREEN RIVER FORMATION) \\
\hline 124GRRV & GREEN RIVER FORMATION \\
\hline 124 PCCK & PARACHUTE CREEK MEMBER (GREEN RIVER FORMATION) \\
\hline $124 \mathrm{TPTG}$ & TIPTON TONGUE (GREEN RIVER FORMATION) \\
\hline 124UINT & UINTA FORMATION \\
\hline $124 \mathrm{WSTC}$ & WASATCH FORMATION \\
\hline 125FRUN & FORT UNION FORMATION \\
\hline $1250 \mathrm{CRK}$ & OHIO CREEK MEMBER (HUNTER CREEK FORMATION) \\
\hline 125PLCN & PLIOCENE SERIES \\
\hline $210 \mathrm{CRCS}$ & CRETACEOUS SERIES \\
\hline 210DKOT & DAKOTA SANDSTONE \\
\hline 211ALMD & ALMOND FORMATION (MESAVERDE GROUP) \\
\hline 211 BLIR & BLAIR FORMATION (MESAVERDE GROUP) \\
\hline 211BXTR & BAXTER SHALE \\
\hline $211 \mathrm{CODY}$ & CODY SHALE \\
\hline $211 \mathrm{CSLG}$ & CASTLEGATE SANDSTONE (MESAVERDE GROUP) \\
\hline 211EMRY & EMERY SANDSTONE MEMBER (MANCOS SHALE) \\
\hline $211 \mathrm{ERCS}$ & ERICSON FORMATION (MESAVERDE GROUP) \\
\hline 211FRLD & FRUITLAND FORMATION \\
\hline 211FRNR & FRONTIER FORMATION \\
\hline 211 FRRN & FERRON SANDSTONE MEMBER (MANCOS SHALE) \\
\hline $211 \mathrm{FXHL}$ & FOX HILLS SANDSTONE \\
\hline $211 H L R D$ & HILLIARD SHALE \\
\hline $211 \mathrm{LNCE}$ & LANCE FORMATION \\
\hline 211 LWIS & LEWIS SHALE \\
\hline $211 \mathrm{MNCS}$ & MANCOS SHALE \\
\hline 211MVRD & MESAVERDE FORMATION \\
\hline 211NBRR & NIOBRARA FORMATION \\
\hline 21 IPCRV & PRICE RIVER FORMATION (MESAVERDE GROUP) \\
\hline $211 \mathrm{RKSP}$ & ROCK SPRINGS FORMATION (MESAVERDE GROUP) \\
\hline 211 STEL & STEELE SHALE \\
\hline 211TRCK & TROUT CREEK SANDSTONE MEMBER (IIES FORMATION) \\
\hline $211 \mathrm{TRPC}$ & TROPIC SHALE \\
\hline $217 \mathrm{ASPN}$ & ASPEN SHALE \\
\hline $217 \mathrm{BCKR}$ & BUCKHORN CONGLOMERATE MEMBER (CEDAR MOUNTAIN FORMATION) \\
\hline $217 \mathrm{BRRV}$ & BEAR RIVER FORMATION \\
\hline $217 \mathrm{CDMN}$ & CEDAR MOUNTAIN FORMATION \\
\hline $217 \mathrm{MDDY}$ & MUDDY SANDSTONE \\
\hline 220GLNC & GLEN CANYON GROUP \\
\hline 22ONGGT & NUGGET SANDSTONE \\
\hline 220 NVJO & NAVAJO SANDSTONE (GLEN CANYON GROUP) \\
\hline 221CRTS & CURIIS FORMATION (SAN RAFAEL GROUP) \\
\hline
\end{tabular}


Table 1.--Geologic codes and formation names--Continued

\begin{tabular}{|c|c|}
\hline CODE & FORMATION NAME \\
\hline 221ENRD & ENTRADA SANDSTONE (SAN RAFAEL GROUP) \\
\hline 221MRSN & MORRISON FORMATION \\
\hline 221SLWS & SALT WASH SANDSTONE MEMBER (MORRISON FORMATION) \\
\hline 221SNDC & SUNDANCE FORMATION \\
\hline $231 \mathrm{CHNL}$ & CHINLE FORMATION \\
\hline 231CRMN & CROW MOUNTAIN SANDSTONE (CHUGWATER GROUP) \\
\hline 231SRMP & SHINARUMP MEMBER (CHINLE FORMATION) \\
\hline 231WNGT & WINGATE SANDSTONE (GLEN CANYON GROUP) \\
\hline 237DNDY & DINWOODY FORMATION \\
\hline 237GSEG & GOOSE EGG FORMATION \\
\hline 237MNKP & MOENKOPI FORMATION \\
\hline 237SNBD & SINBAD LIMESTONE MEMBER (MOENKOPI FORMATION) \\
\hline 237TYNS & THAYNES LIMESTONE \\
\hline 237 WDSD & WOODSIDE SHALE \\
\hline 310PMPV & PERMIAN-PENNSYLVANIAN SYSTEM \\
\hline $310 \mathrm{PRMN}$ & PERMIAN SYSTEM \\
\hline 310WEBR & WEBER SANDSTONE \\
\hline 311PSPR & PHOSPHORIA FORMATION \\
\hline $317 \mathrm{CDRM}$ & CEDAR MESA SANDSTONE MEMBER (CUTLER FORMATION) \\
\hline 317CTLR & CUTLER FORMATION \\
\hline 317DCLL & DECHELLY SANDSTONE (CUTLER FORMATION) \\
\hline $317 E L P C$ & ELEPHANT CANYON FORMATION \\
\hline $317 \mathrm{KIBB}$ & KAIBAB LIMESTONE \\
\hline $3170 \mathrm{GRK}$ & ORGAN ROCK TONGUE (CUTLER FORMATION) \\
\hline $317 \mathrm{PRKC}$ & PARK CITY FORMATION \\
\hline 317WTRM & WHITE RIM SANDSTONE MEMBER (CUTLER FORMATION) \\
\hline 320PSLV & PENNSYLVANIAN SYSTEM \\
\hline 321HKTL & HONAKER TRAIL FORMATION (HERMOSA GROUP) \\
\hline 321 TSLP & TENSLEEP SANDSTONE \\
\hline 324HRMS & HERMOSA FORMATION \\
\hline $324 \mathrm{MNRN}$ & MINTURN FORMATION \\
\hline 324MOLS & MOLAS FORMATION \\
\hline 324MRGN & MORGAN FORMATION \\
\hline 324MRON & MAROON FORMATION \\
\hline $324 \mathrm{PKTL}$ & PINKERTON TRAIL FORMATION (HERMOSA GROUP) \\
\hline 324PRDX & PARADOX FORMATION (HERMOSA GROUP) \\
\hline $324 \mathrm{RICO}$ & RICO FORMATION \\
\hline 330MSSP & MISSISSIPPIAN SYSTEM \\
\hline 330RDLL & REDWALL LIMESTONE \\
\hline 331LDVL & LEADVILLE LIMESTONE \\
\hline $331 \mathrm{MDSN}$ & MADISON LIMESTONE \\
\hline $340 \mathrm{DVNN}$ & DEVONIAN SYSTEM \\
\hline $341 \mathrm{ANTH}$ & ANETH FORMATION \\
\hline $341 E L B R$ & ELBERT FORMATION \\
\hline 360ODVC & ORDOVICIAN SYSTEM \\
\hline $370 \mathrm{CMBR}$ & CAMBRIAN SYSTEM \\
\hline
\end{tabular}

${ }^{1}$ Tertiary system undifferentiated included in table 2 . 
HEADINGS FOR TABLES 2-11:

DRILL-STEM TEST DATA

\author{
LOCATION - Township, Range, Section (see fig. 2) \\ STATE - Abbreviated by U.S. Postal Code \\ COUNTY - County name \\ FORMATION - For formation code see table 1 \\ INTERVAL TESTED - Depth in feet below measuring point \\ SHUT-IN HEAD, FT - Head in feet above sea level \\ **** - Data not available \\ ALT. OF M.P. - Altitude of Measuring Point - Altitudes reported \\ by drillers on petroleum logs may be measured \\ from undefined points at land surface, drill \\ floor, Kelly bushing or rotary table. \\ PERMEABILITY - The millidarcy (md) is a standard unit of perme- \\ ability. One darcy is equivalent to the passage \\ of one cubic centimeter of fluid of one centipoise \\ viscosity flowing in one second under a pressure \\ differential of one atmosphere through a porous \\ medium having an area cross-section of one square \\ centimeter. One millidarcy (md) is one one- \\ thousandth of a darcy. The absolute unit of \\ viscosity, poise, is equal to one dyne-second per \\ square centimeter. Centipoise (cp), one one- \\ hundredth of a poise, is a more convenient unit, and \\ the one more commonly used. \\ HYDRAULIC CONDUCTIVITY - In units of feet per day $(\mathrm{ft} / \mathrm{d})$. Values rounded \\ by the computer to 0.00 indicate value is less than \\ $0.01 \mathrm{ft} / \mathrm{d}$. \\ TEMPERATURE $\left({ }^{\circ} \mathrm{F}\right)$ - In degrees Fahrenheit \\ TEST DATE - Year dri11-stem test conducted
}




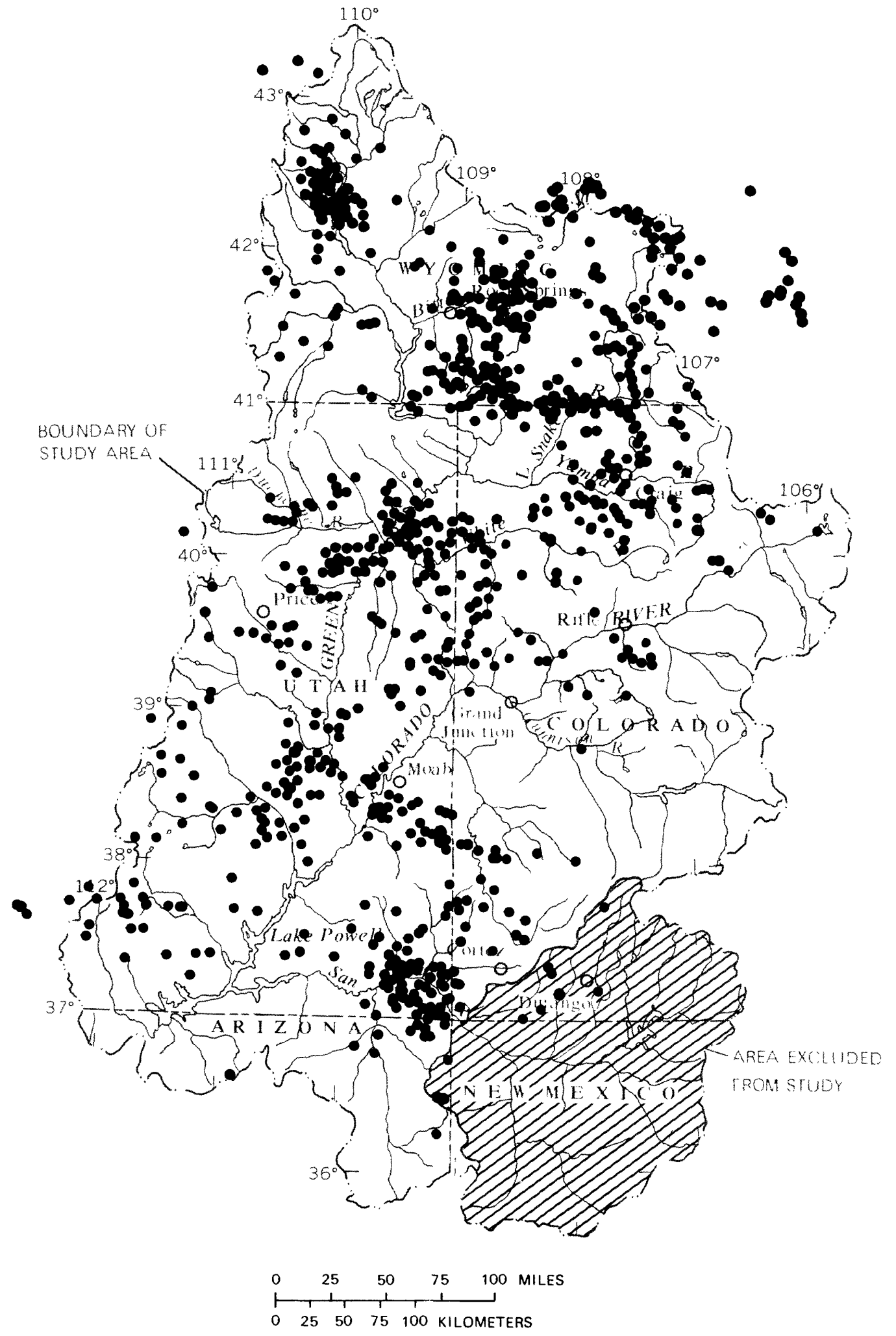

Figure 5.--Location of drill-stem test data for all hydrogeologic units. 


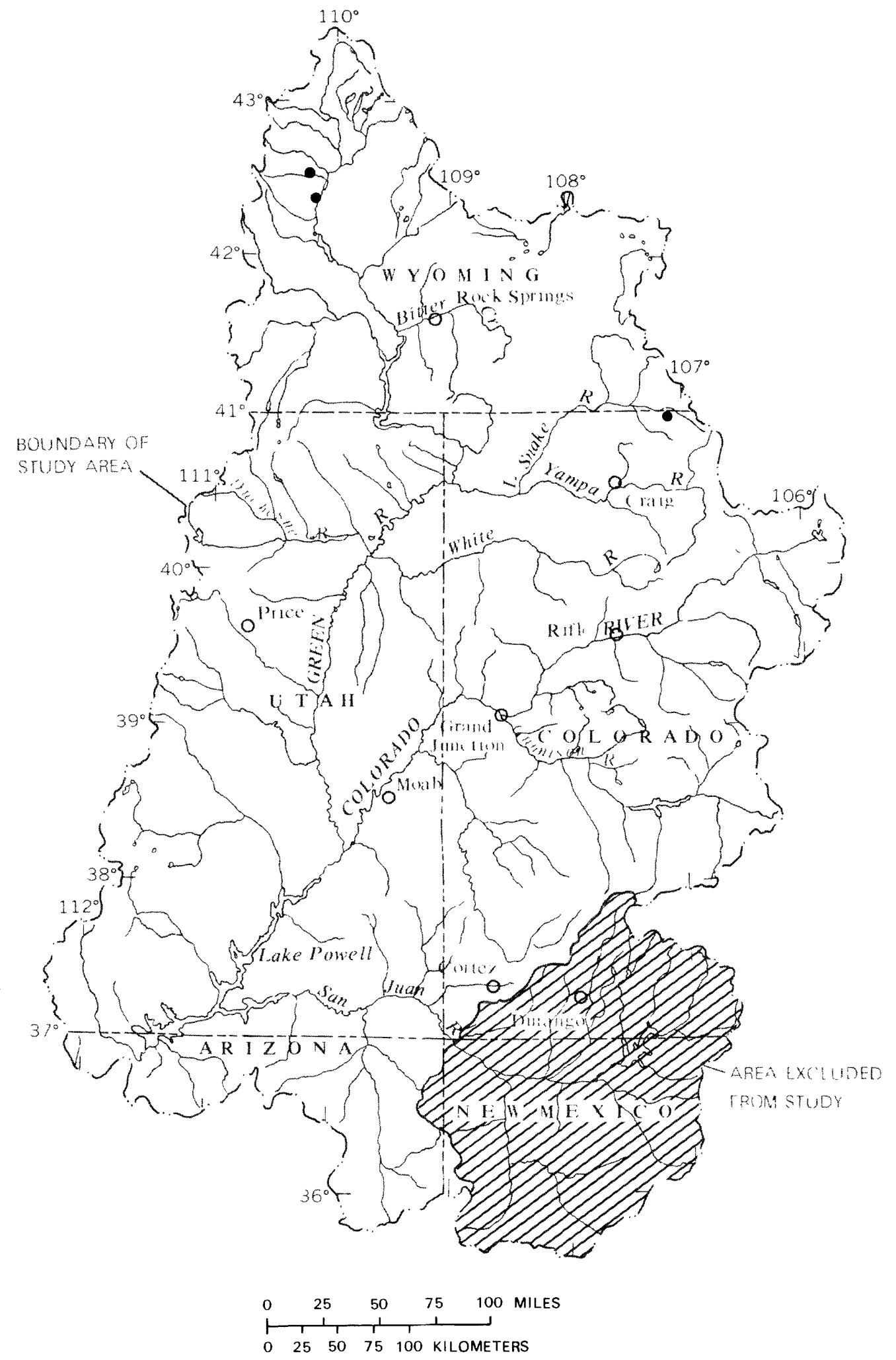

Figure 6.--Location of dri11-stem test data for upper Tertiary discontinuous aquifers. 


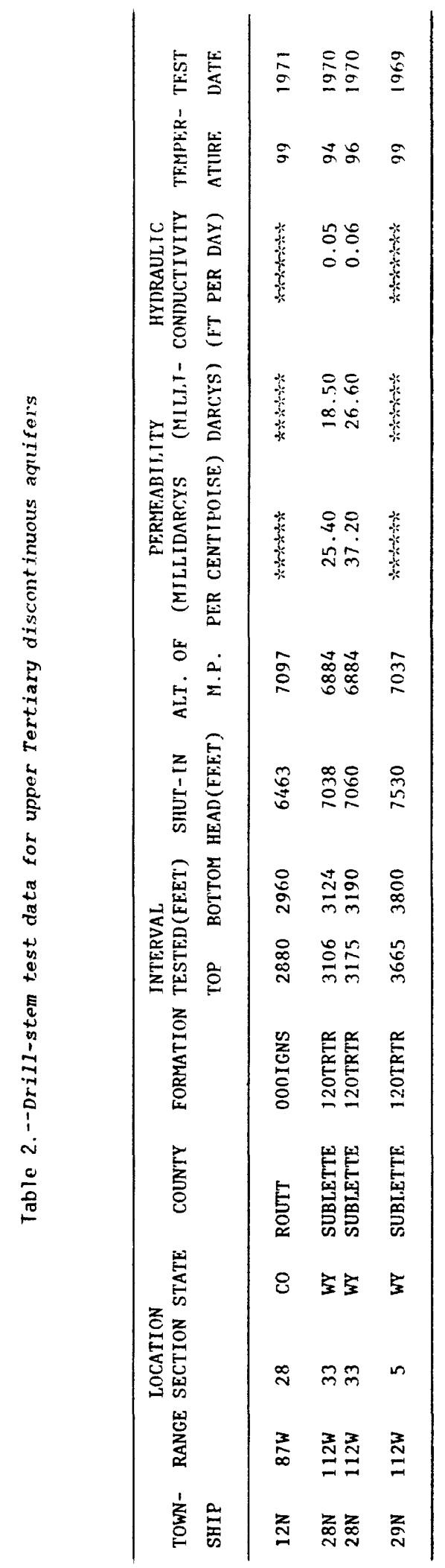




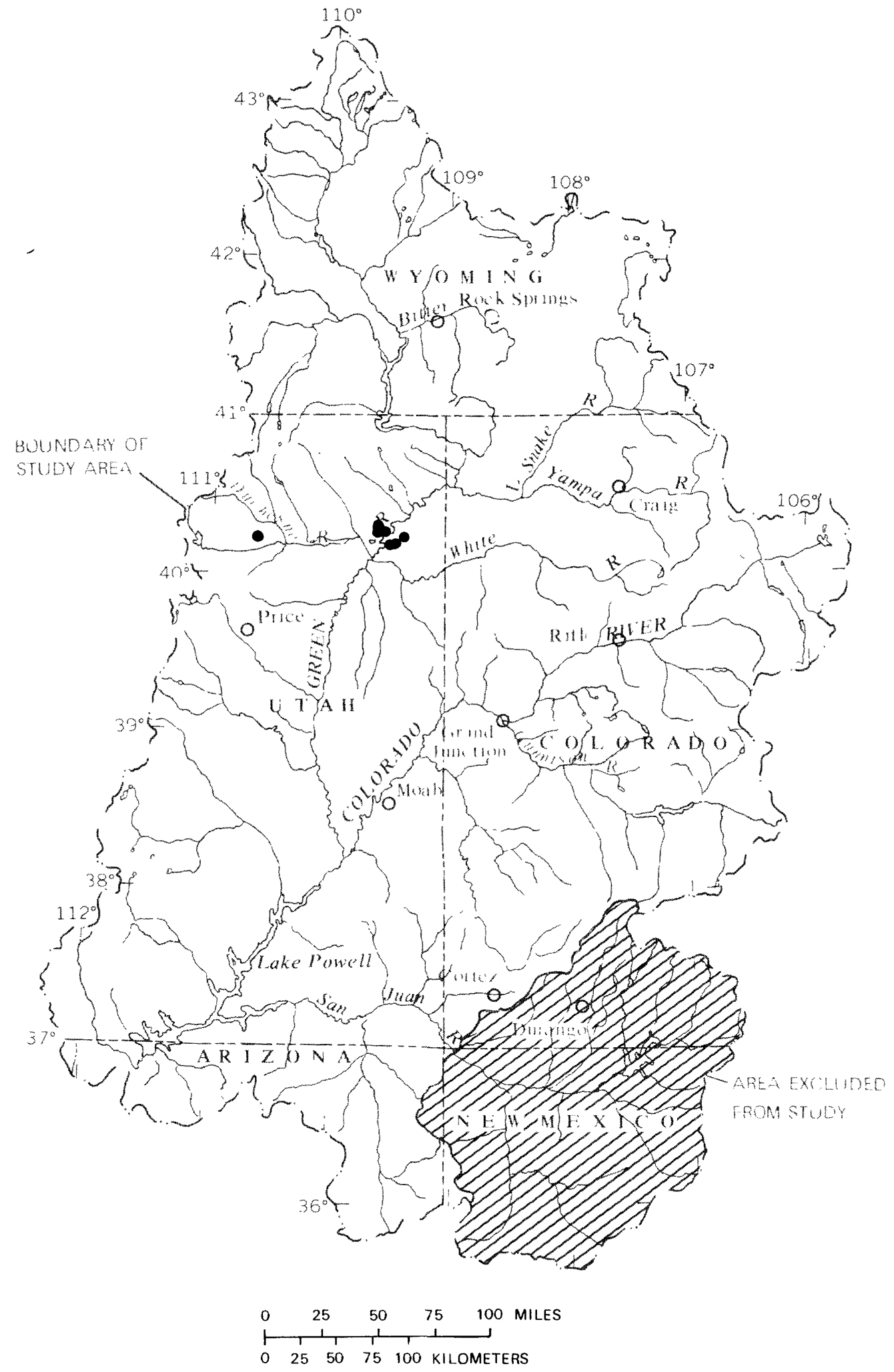

Figure 7.--Location of dri11-stem test data for middle Tertiary aquifers, partly drained aquifers, and confining layers. 


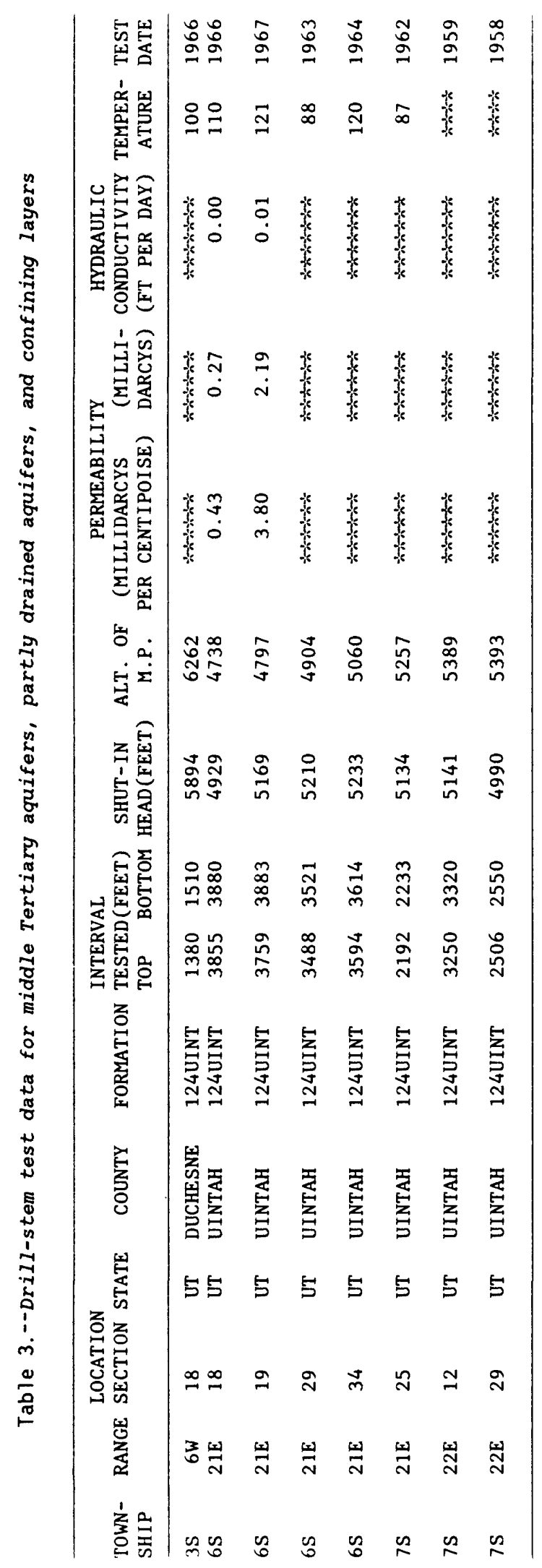




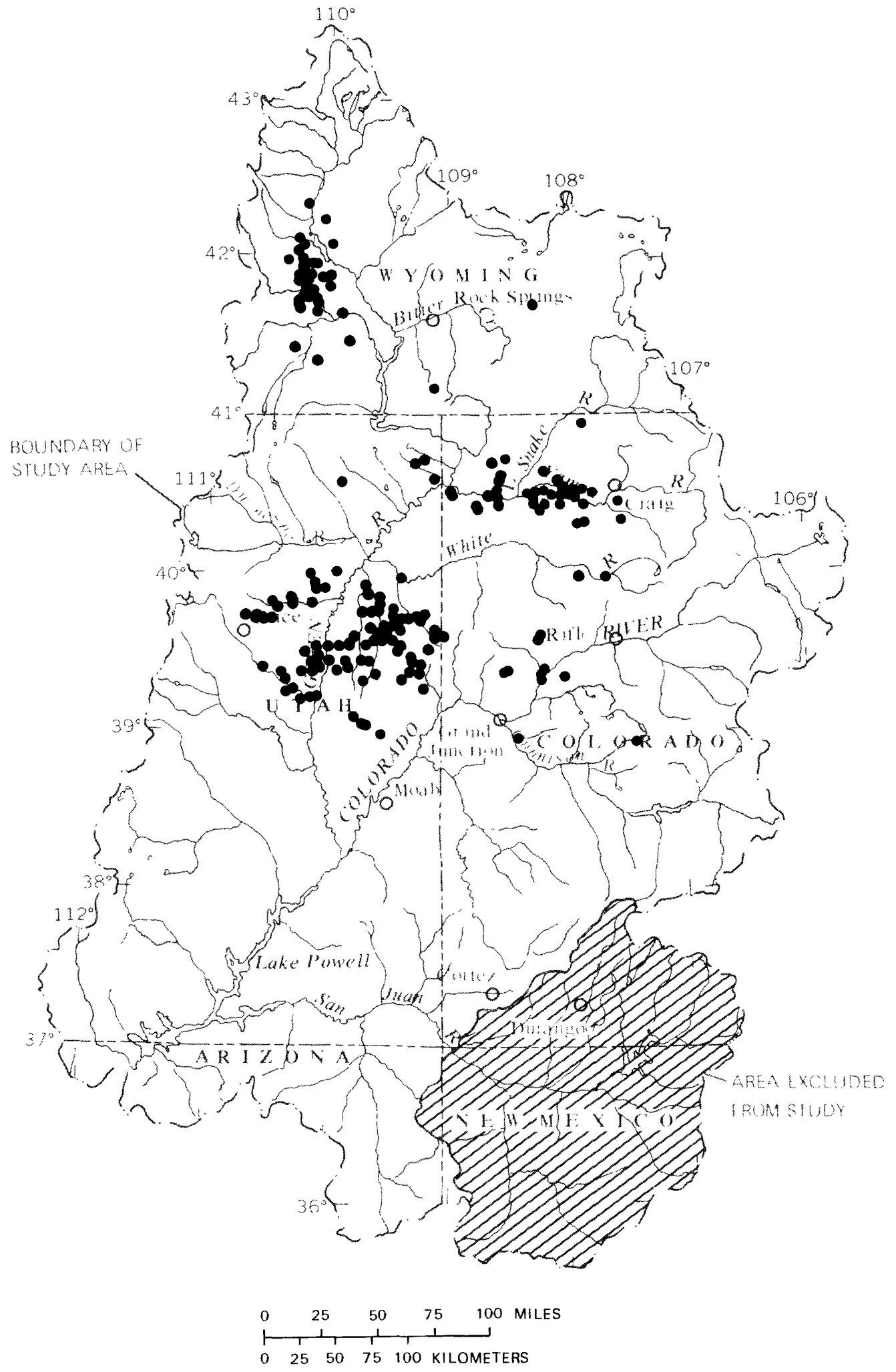

Figure 8.--Location of dri11-stem test data for lower Tertiary aquifers and confining layers. 


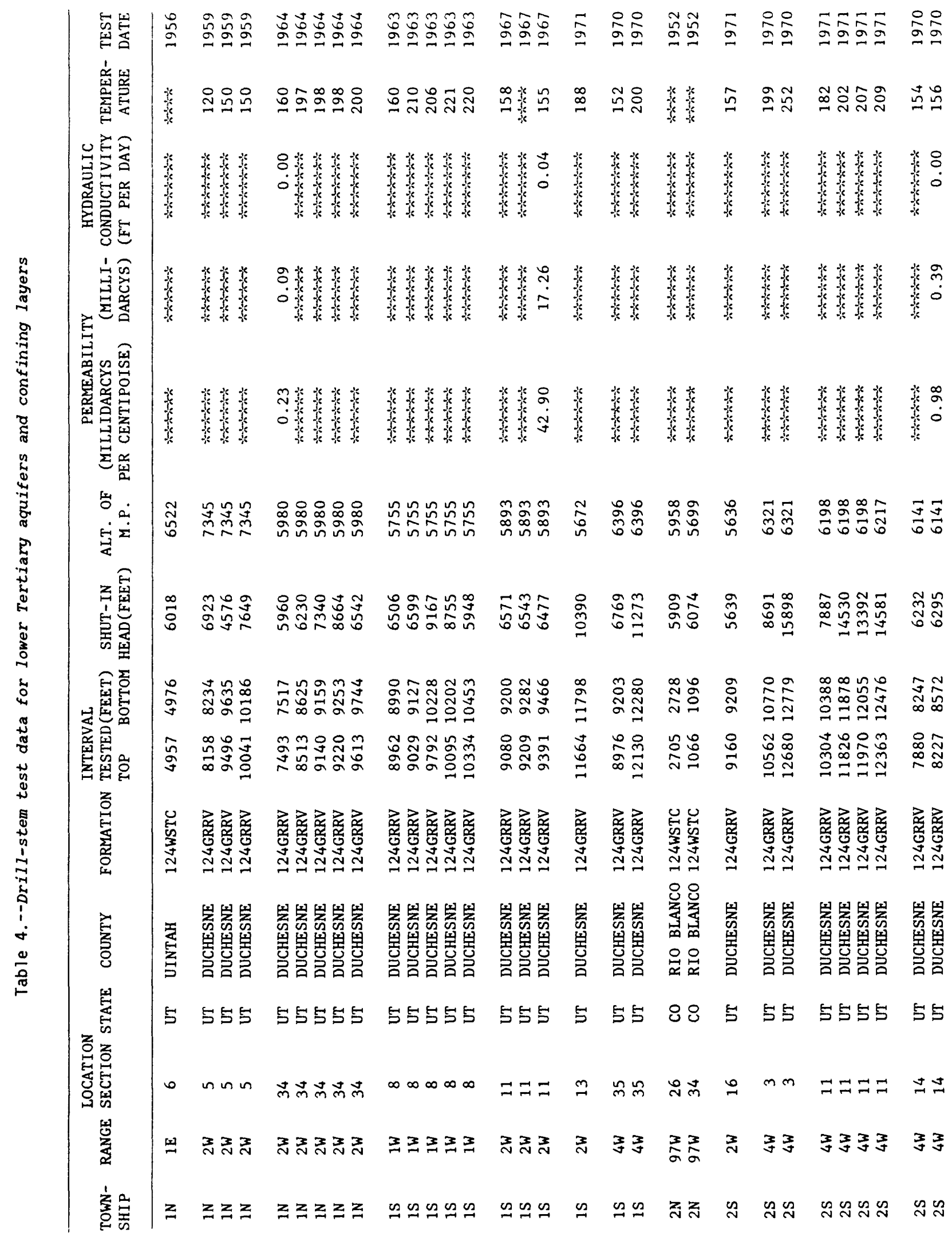




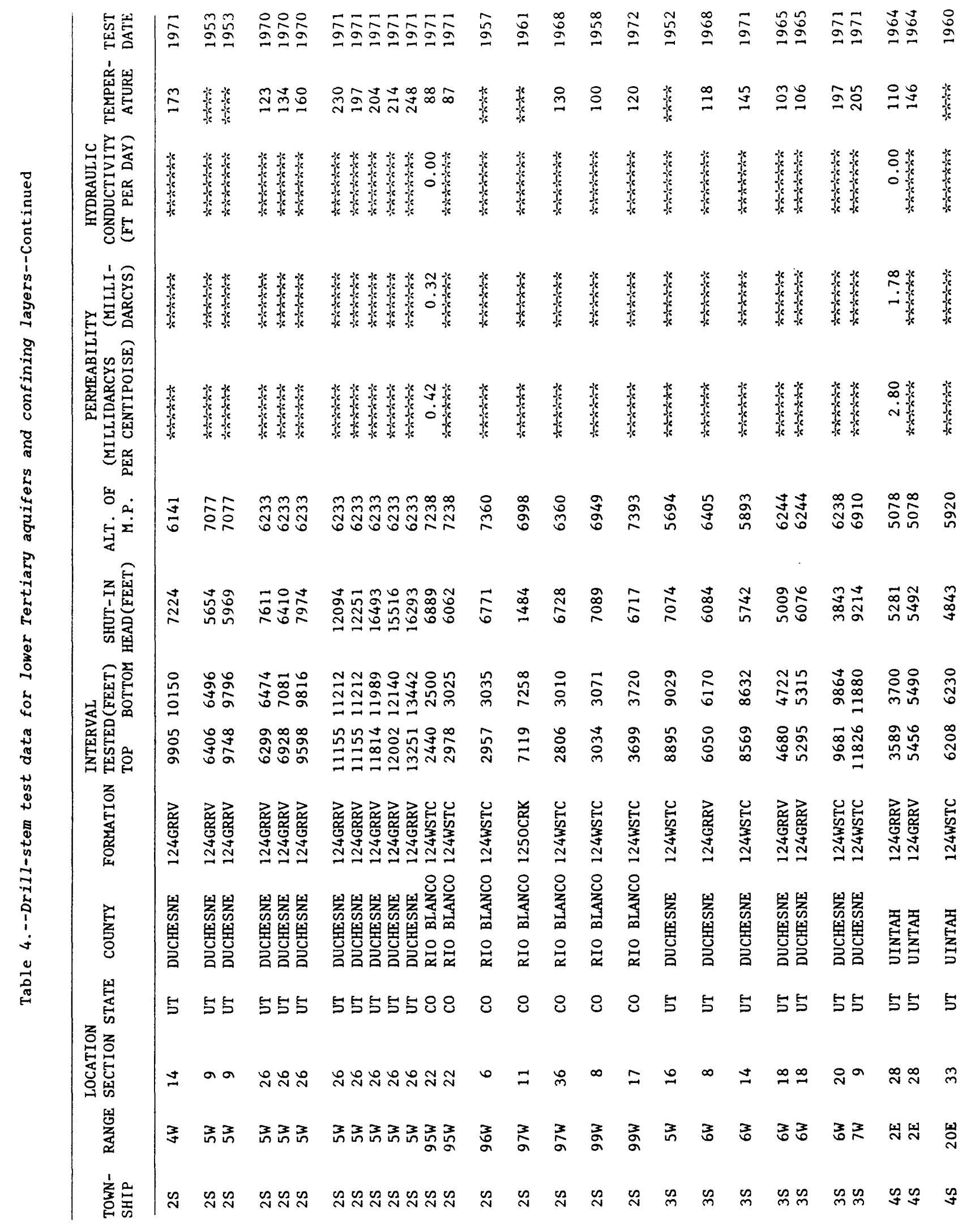




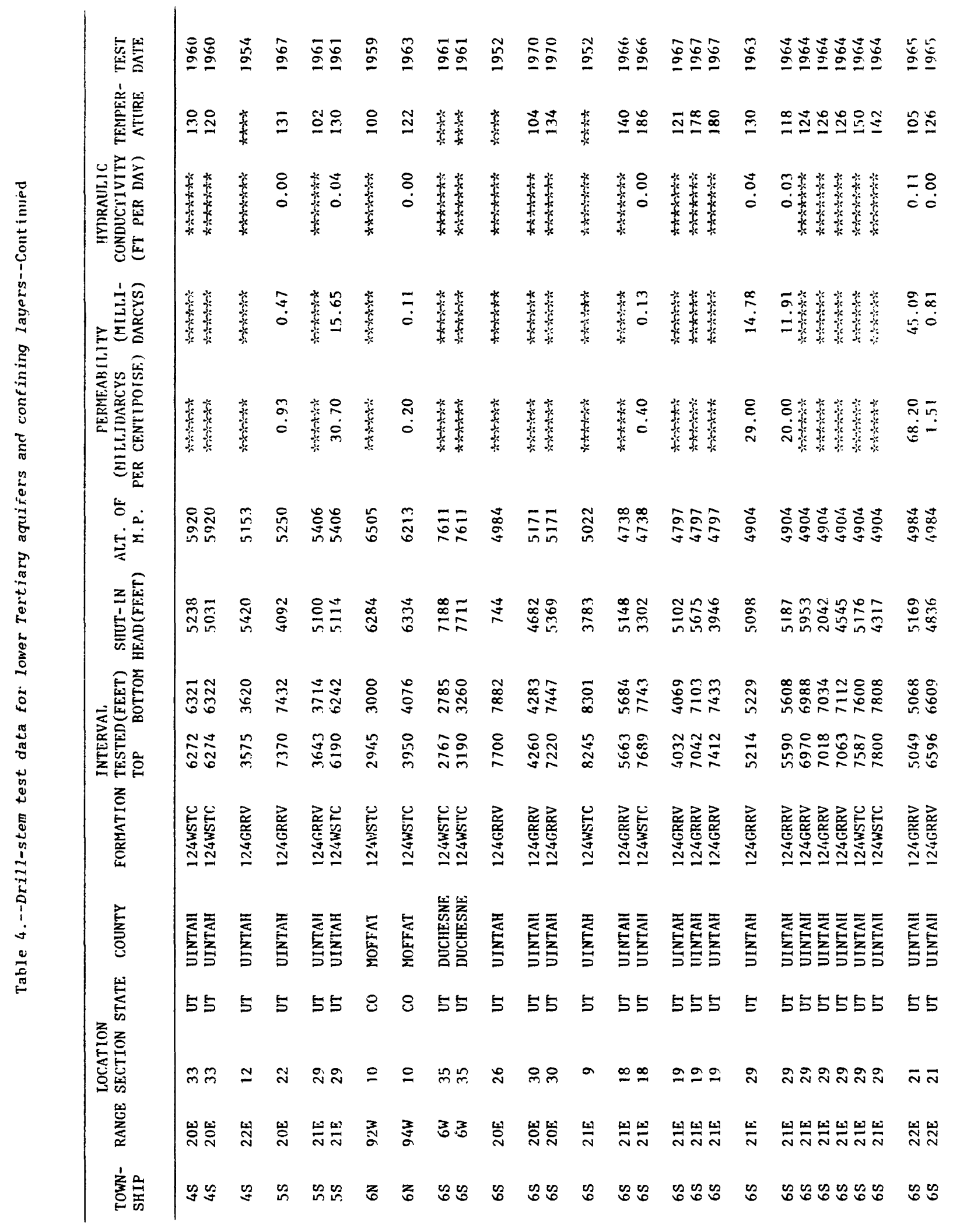




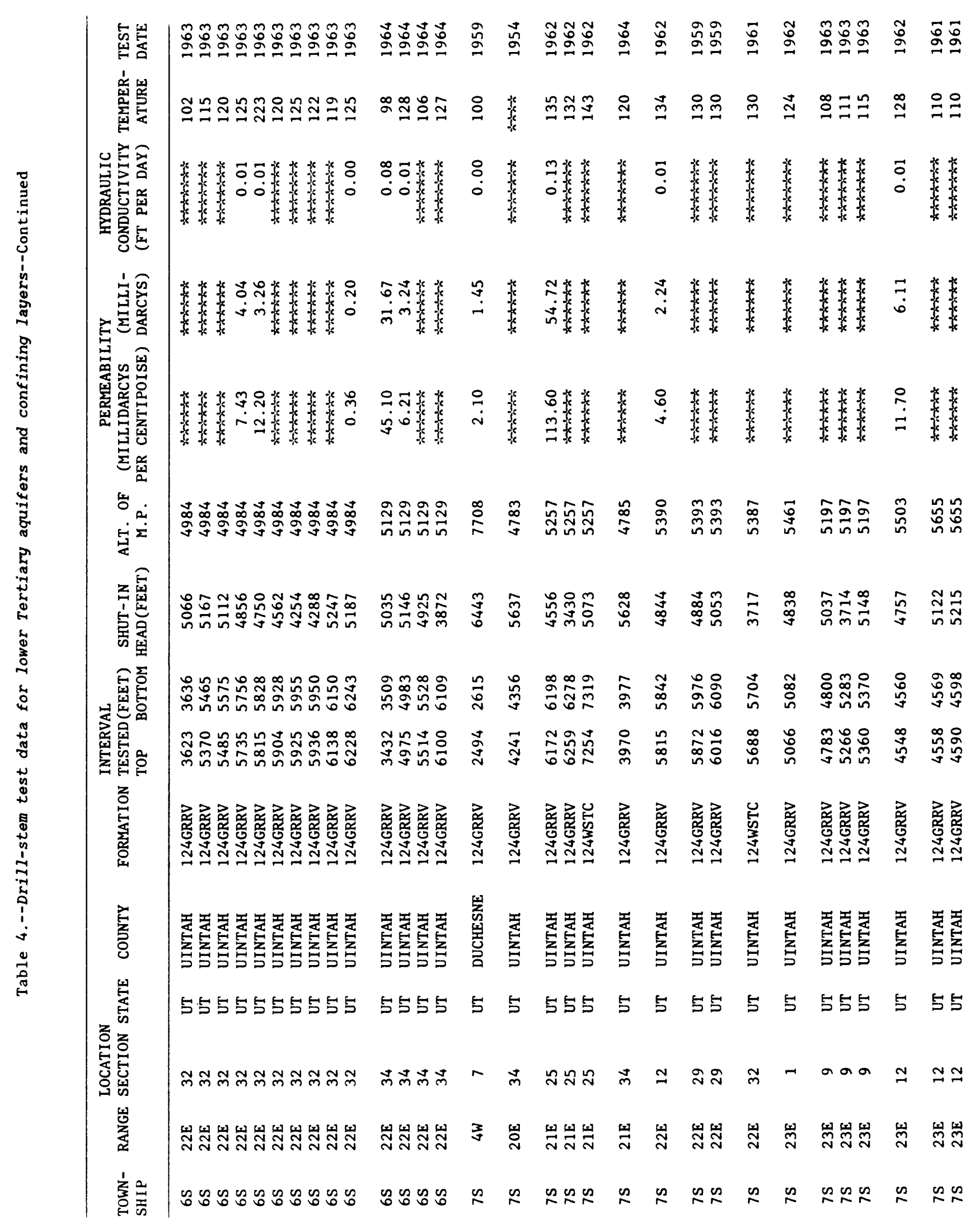




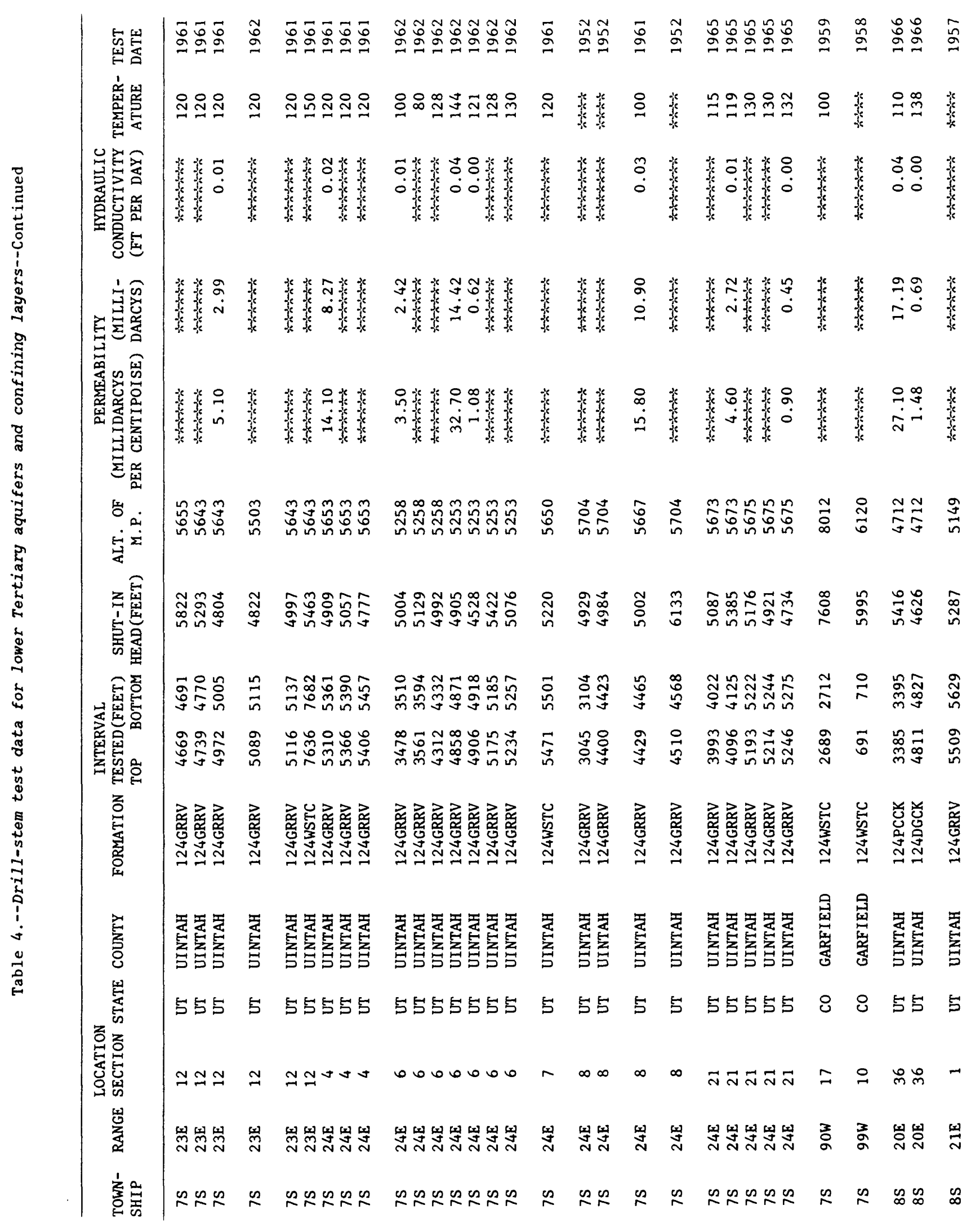




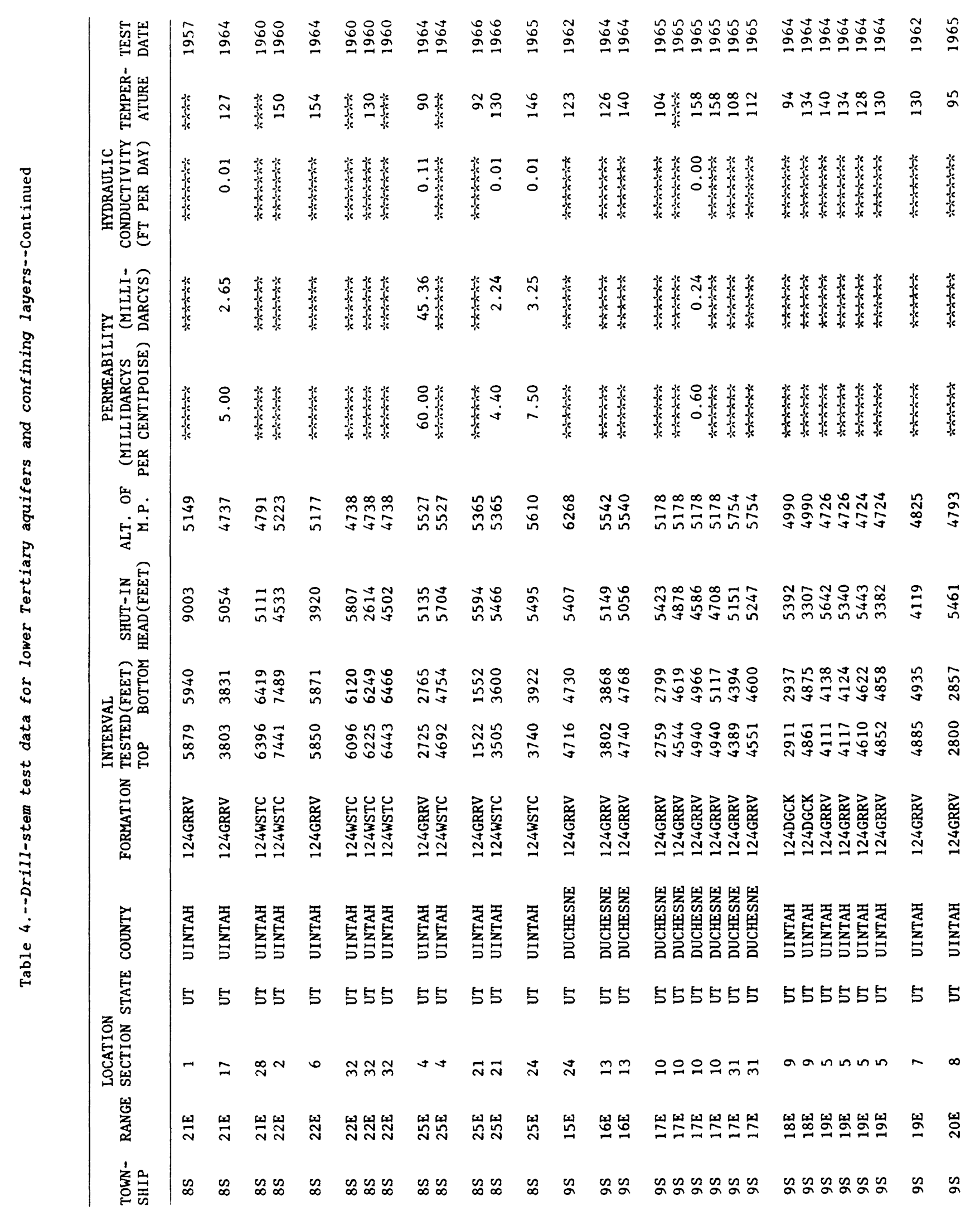




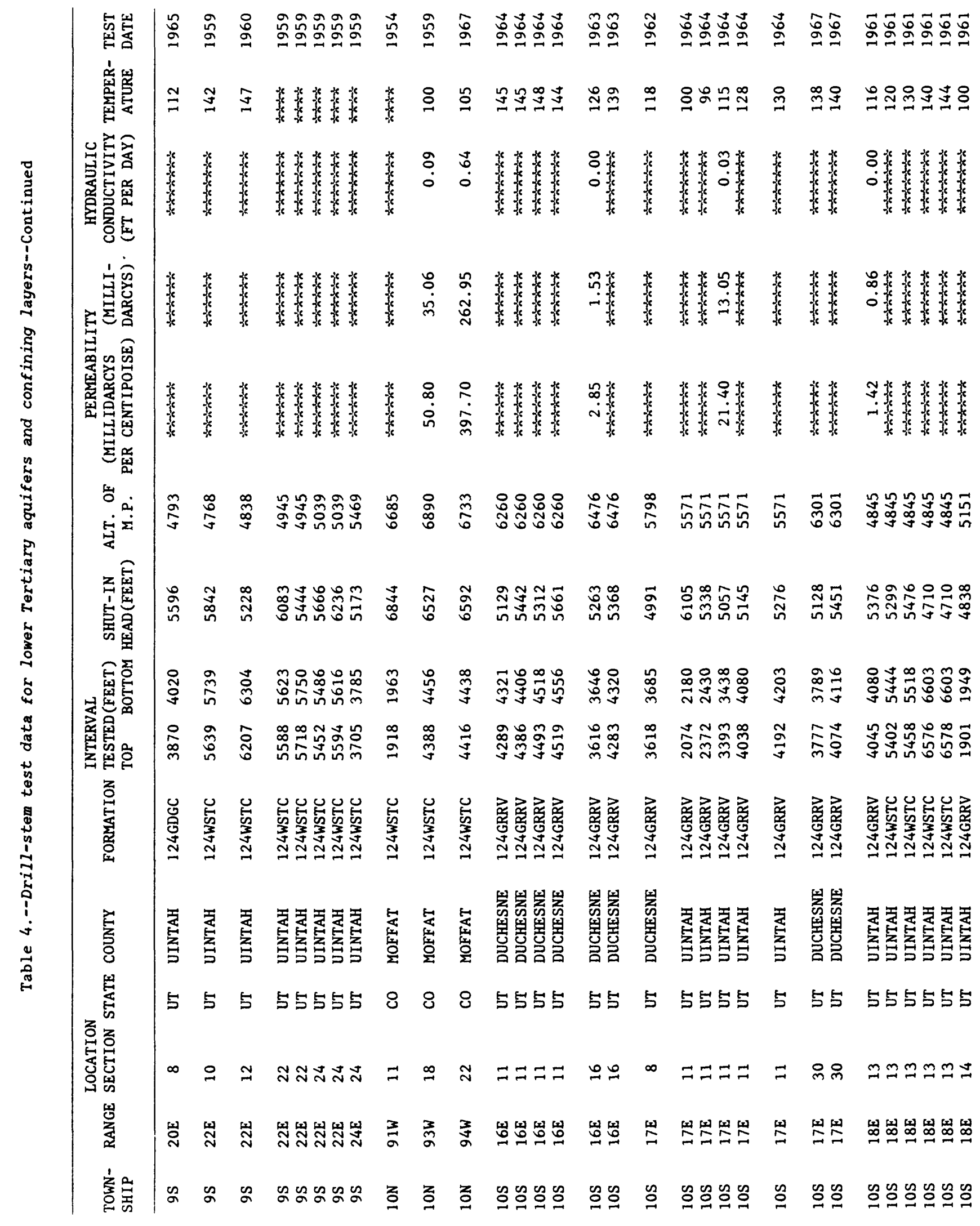




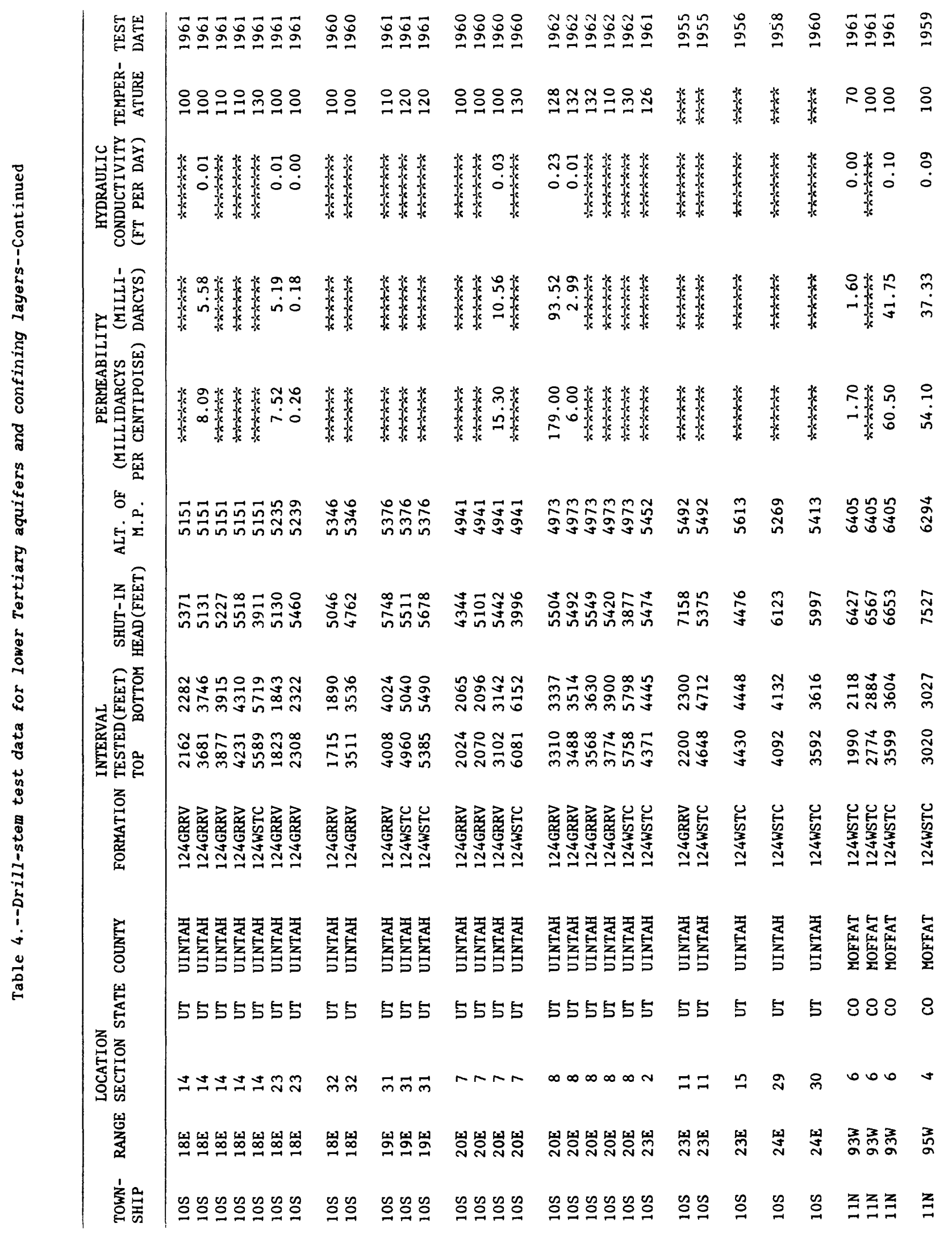




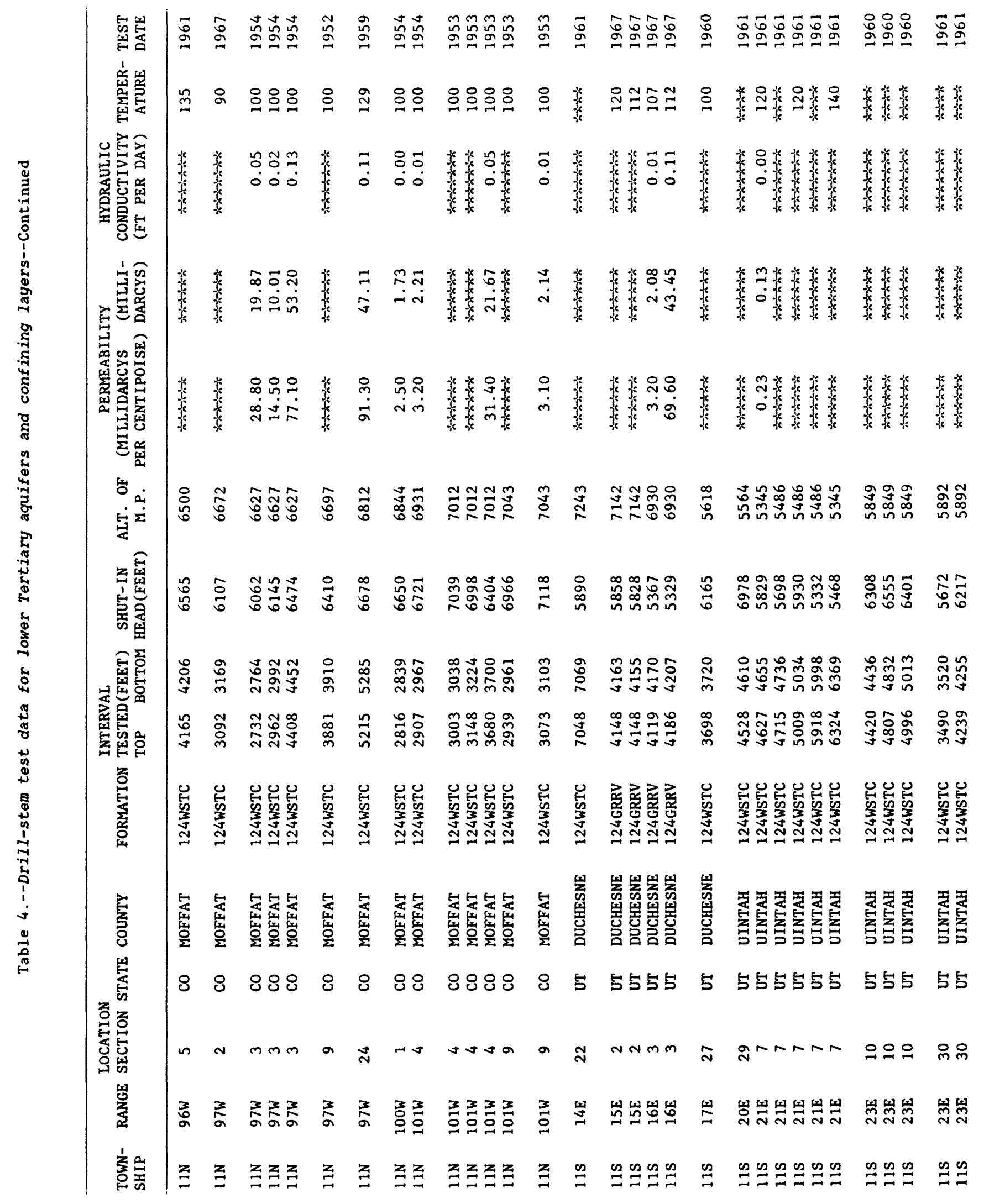




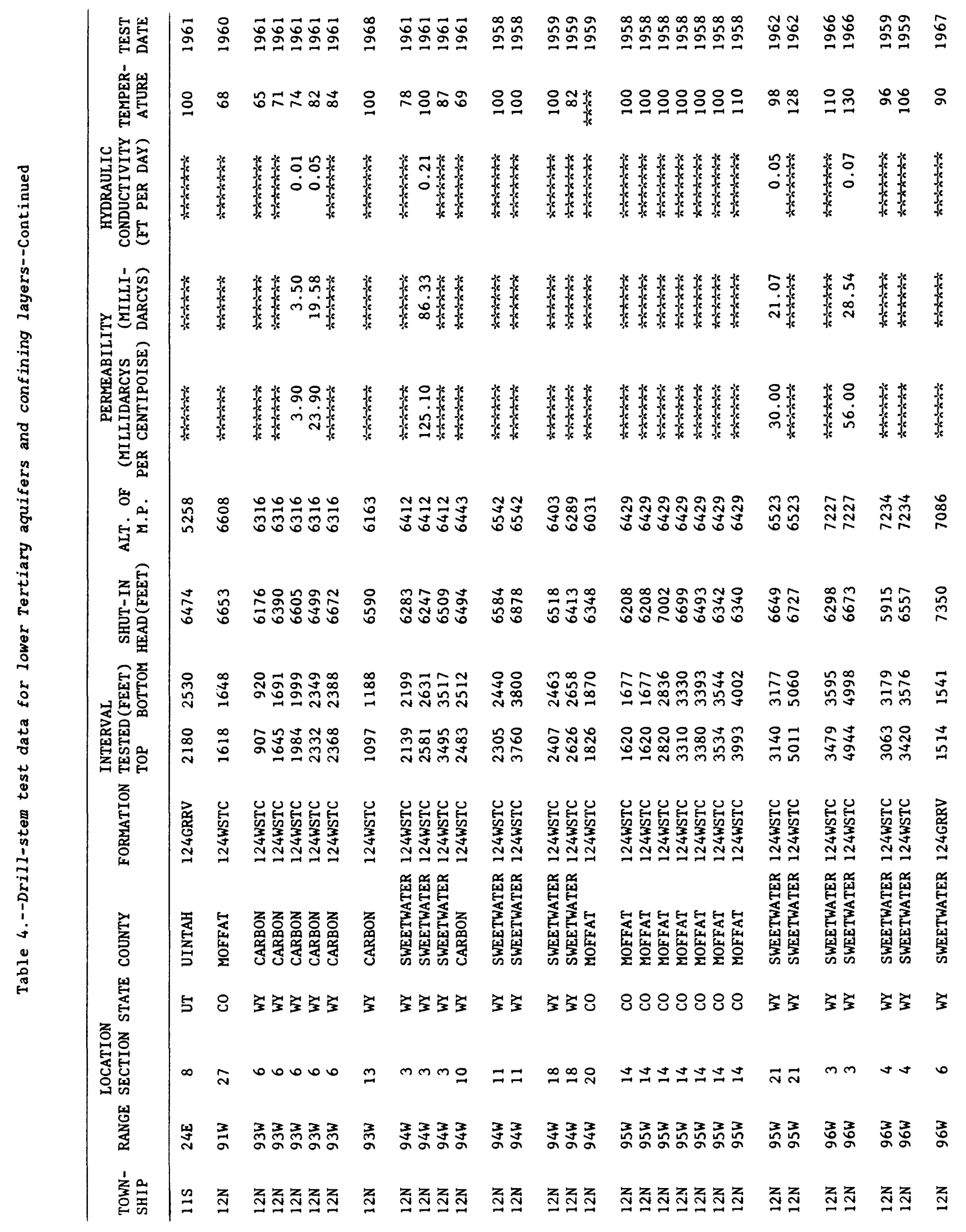




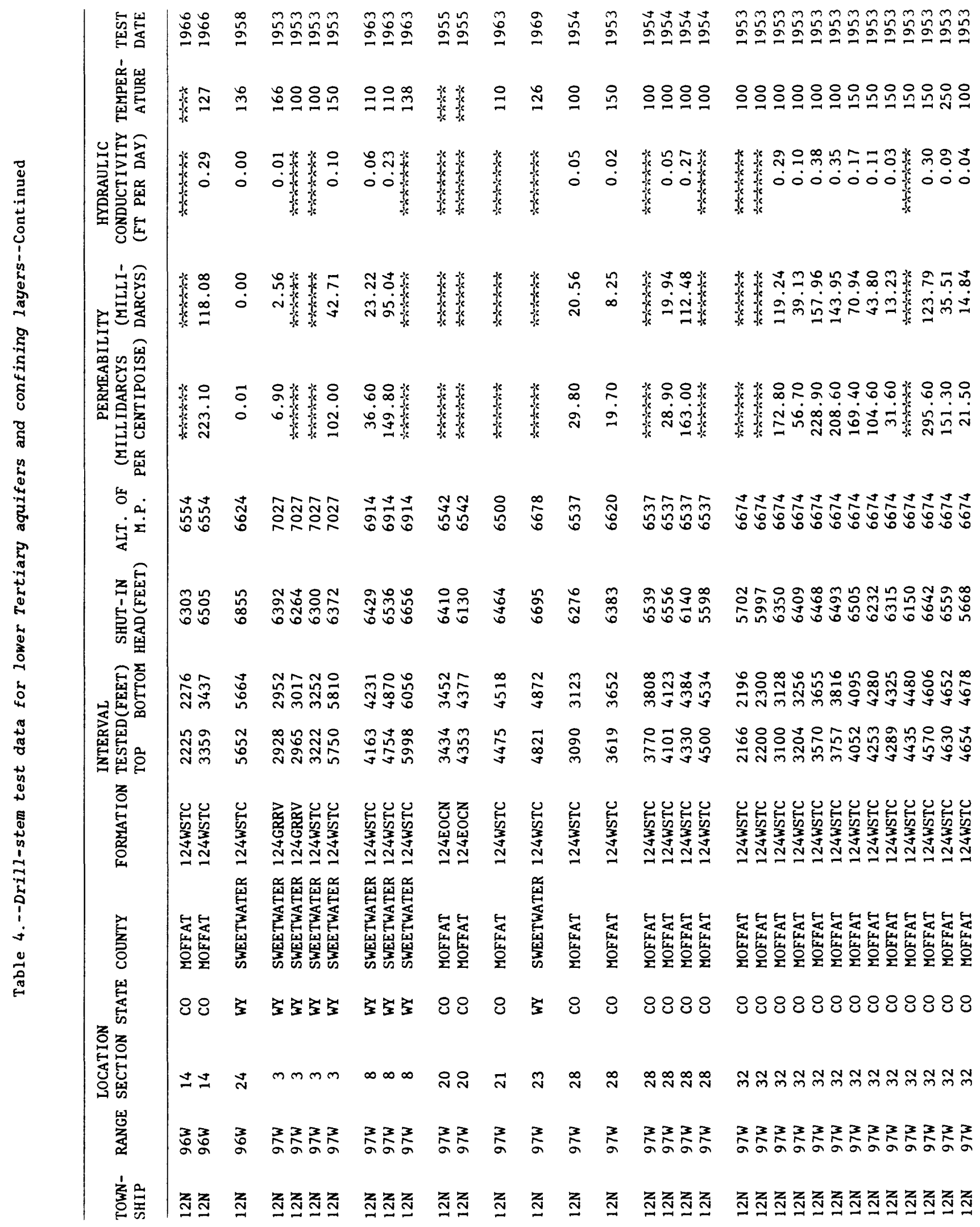




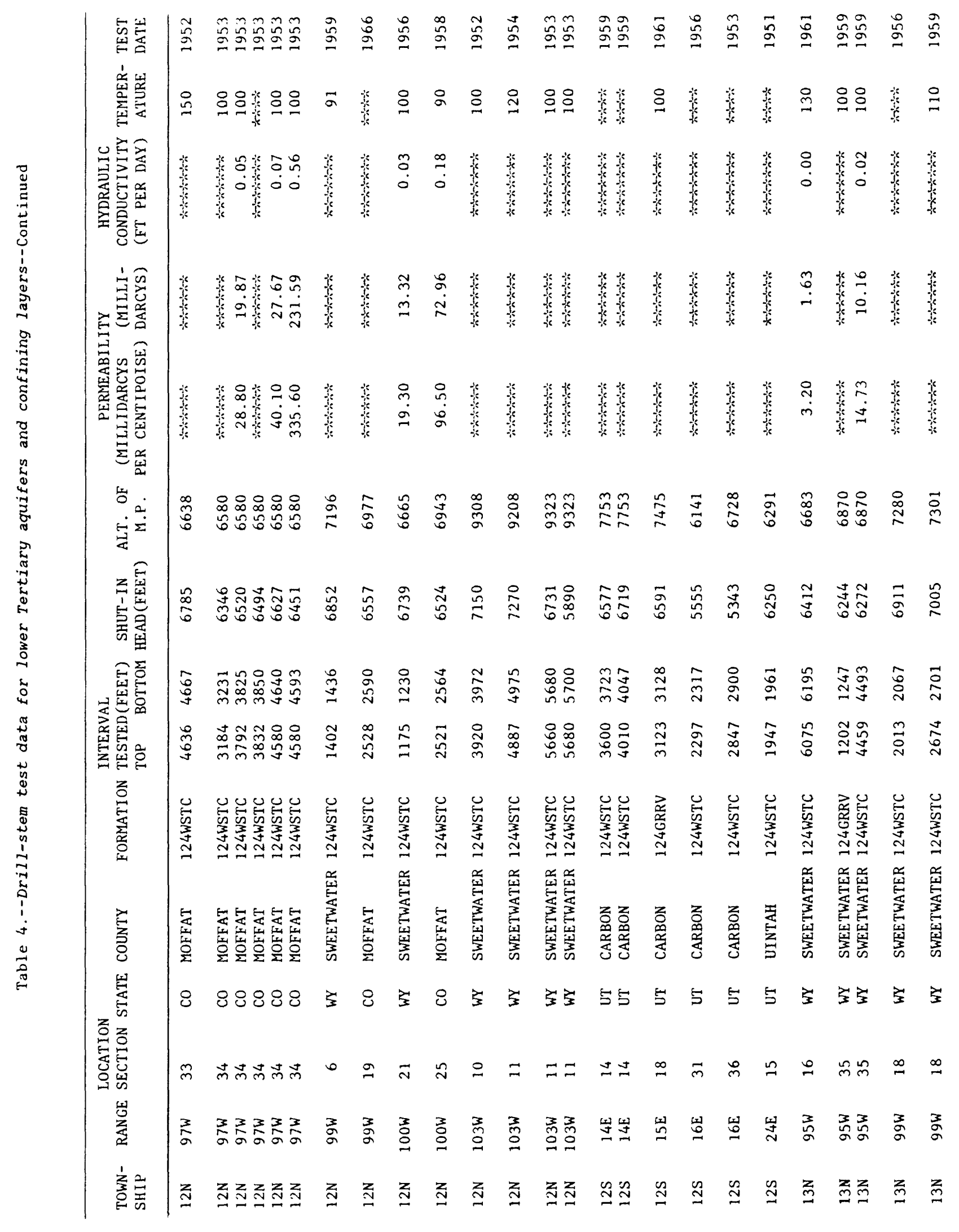




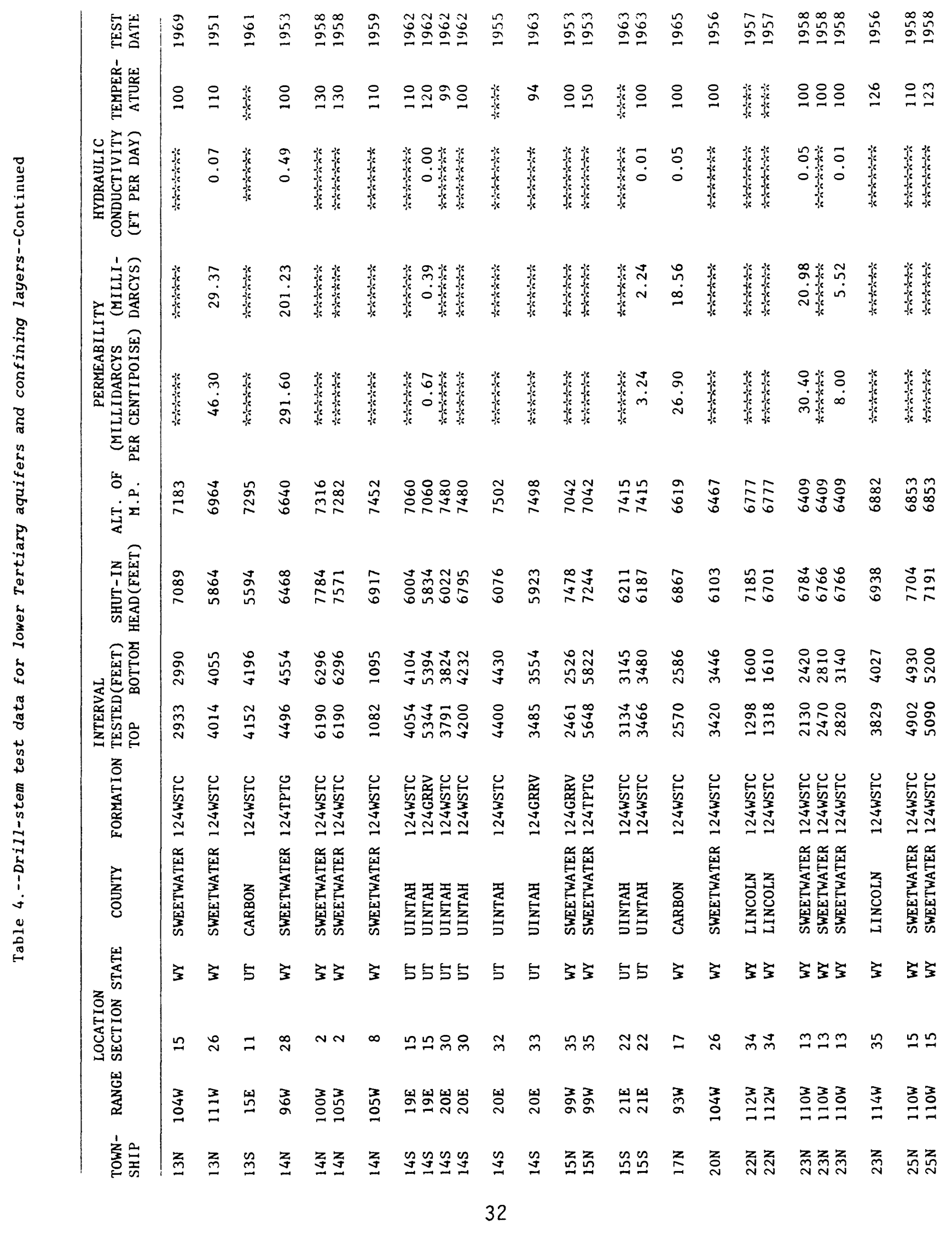




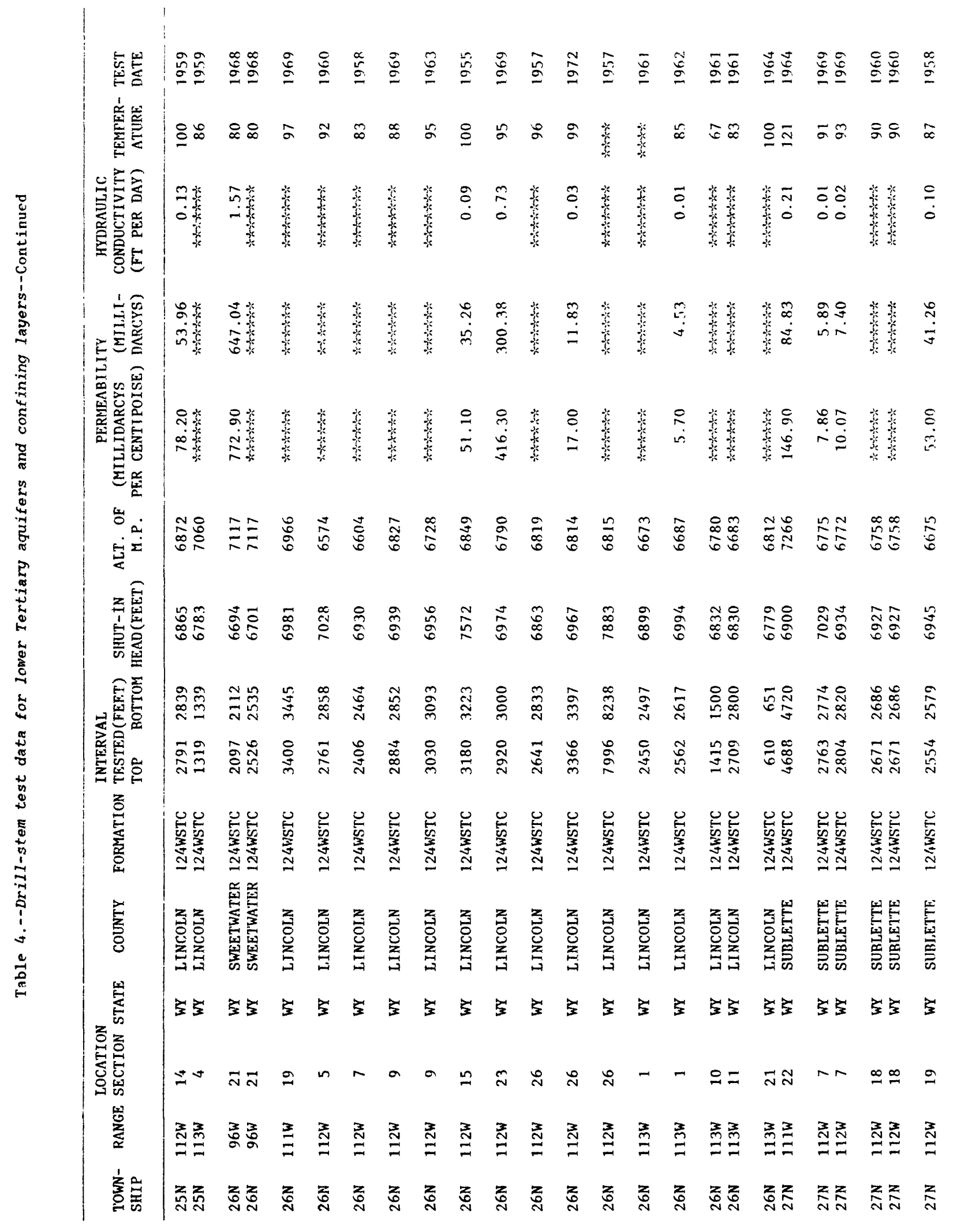




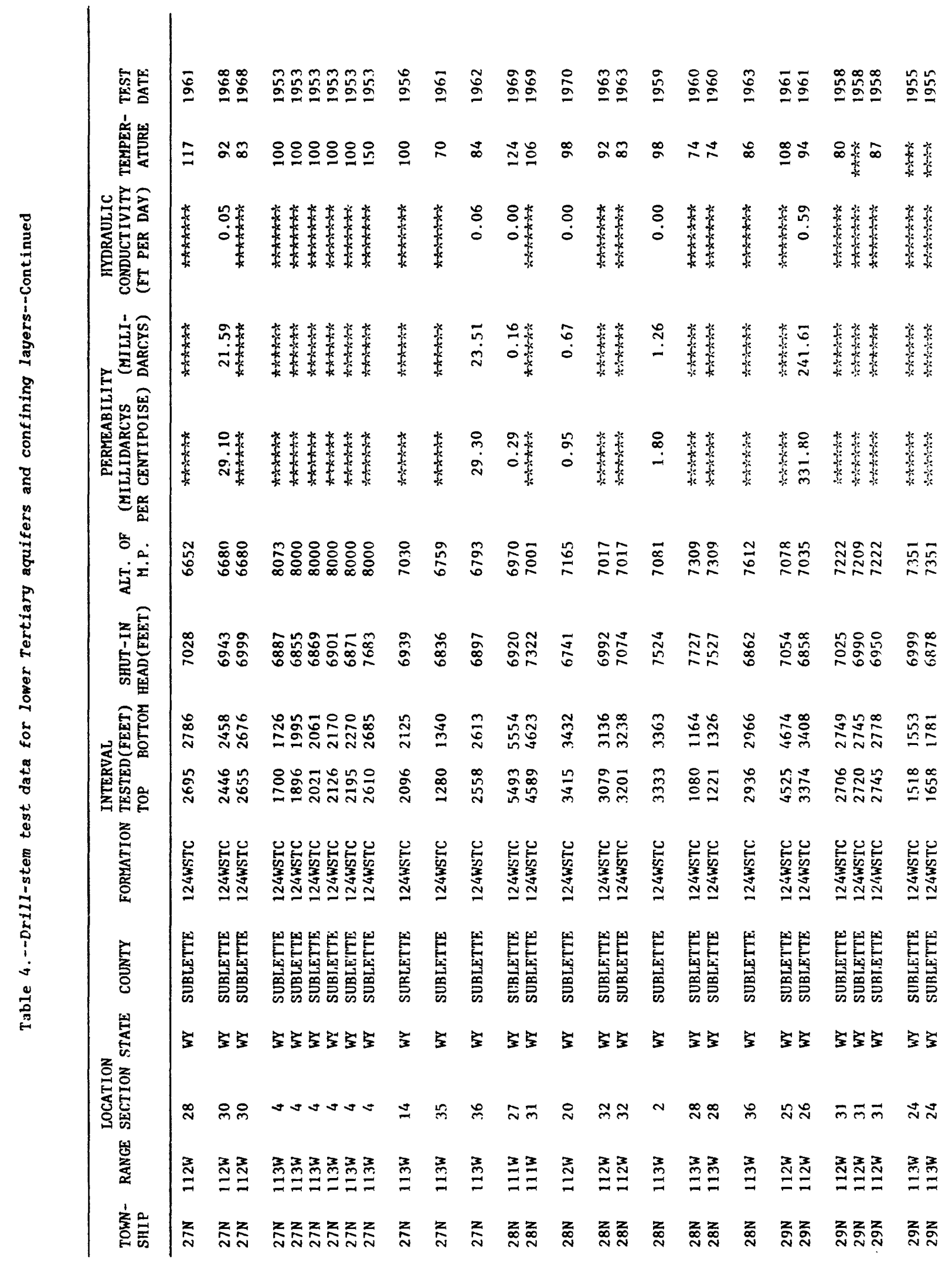




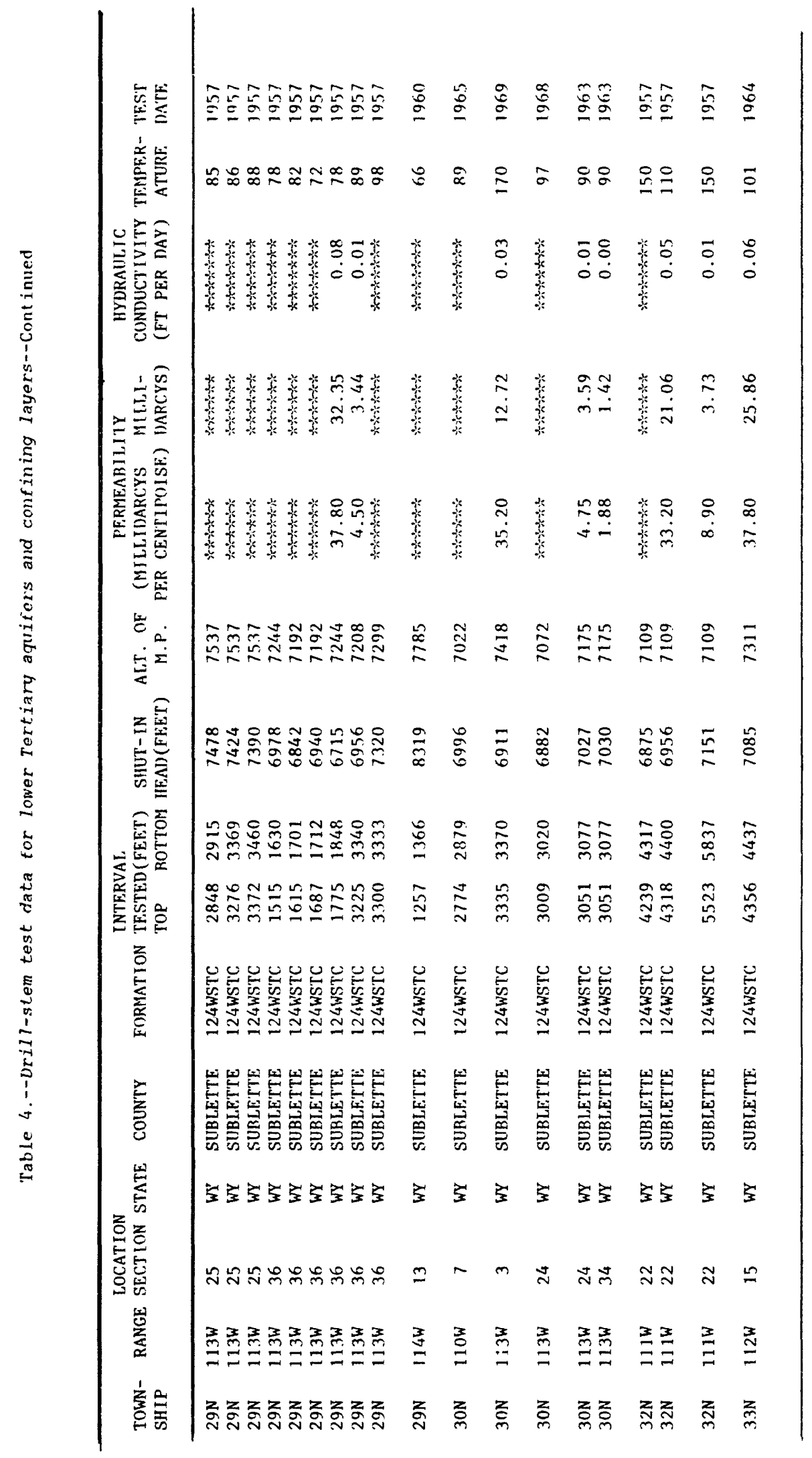




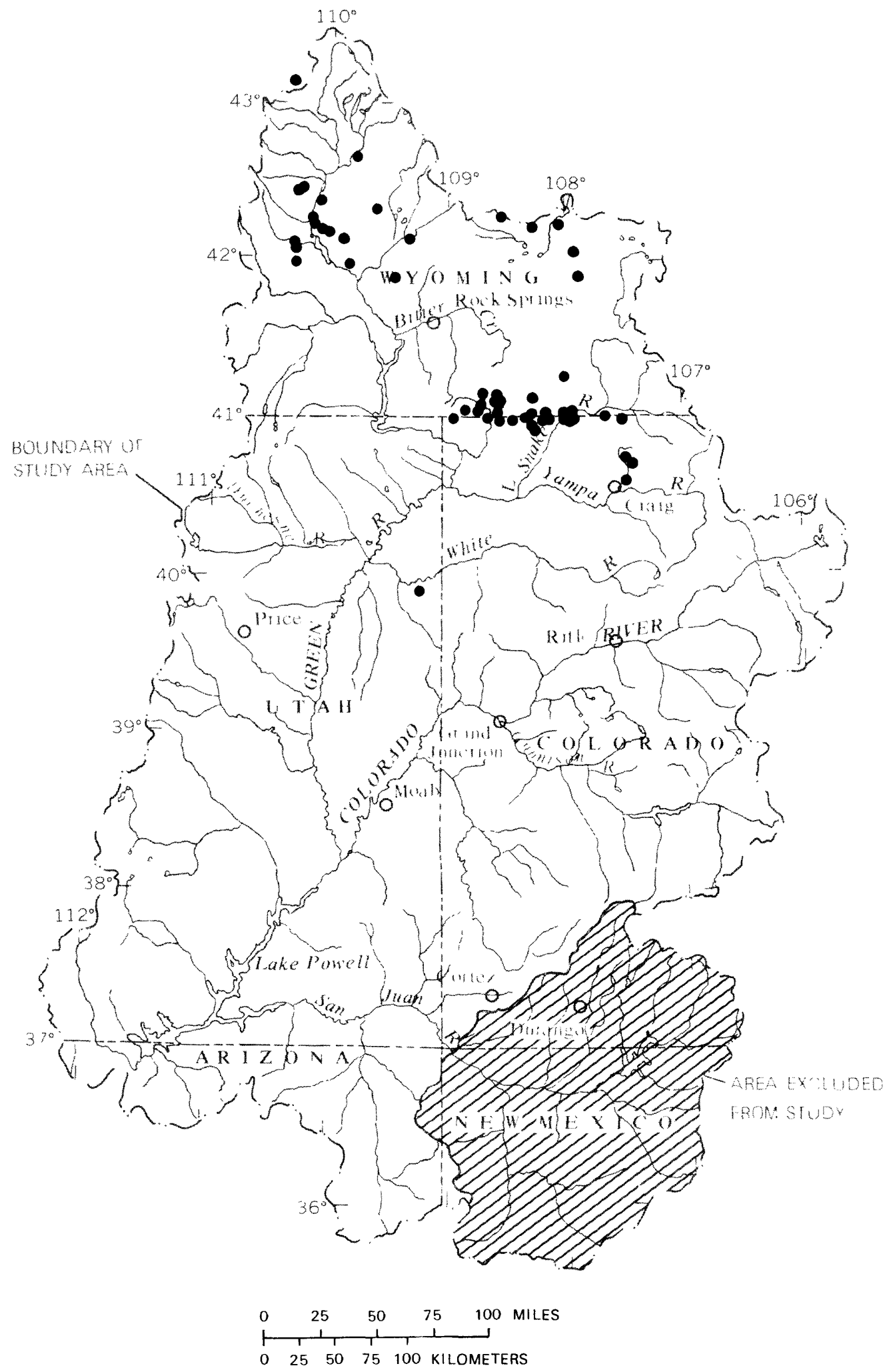

Figure 9.--Location of drill-stem test data for basal Tertiary aquifers. 


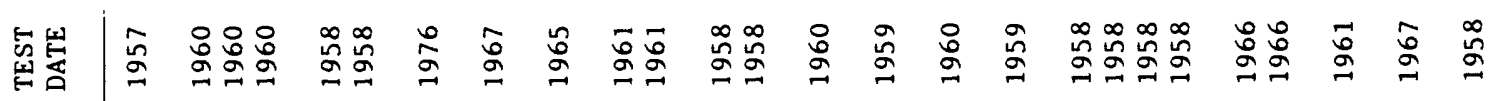

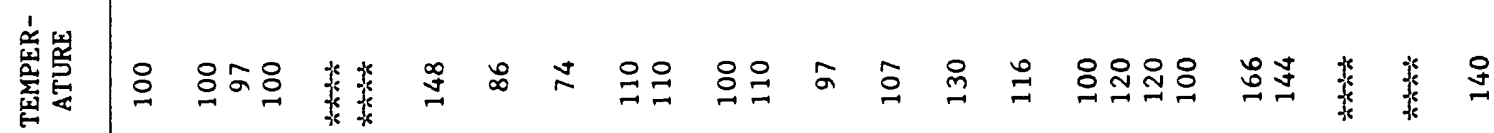

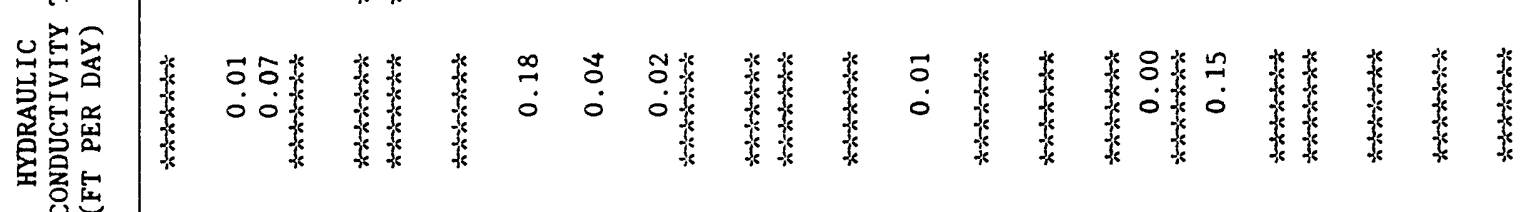

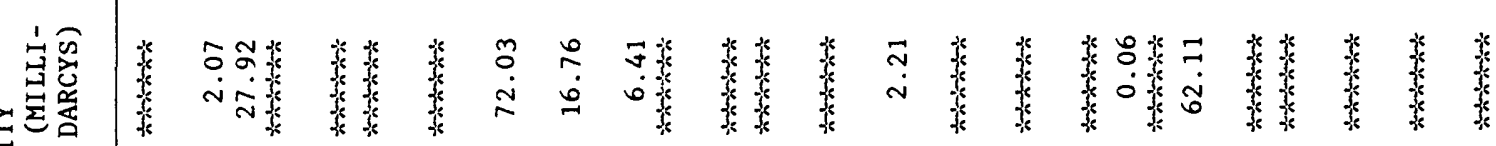

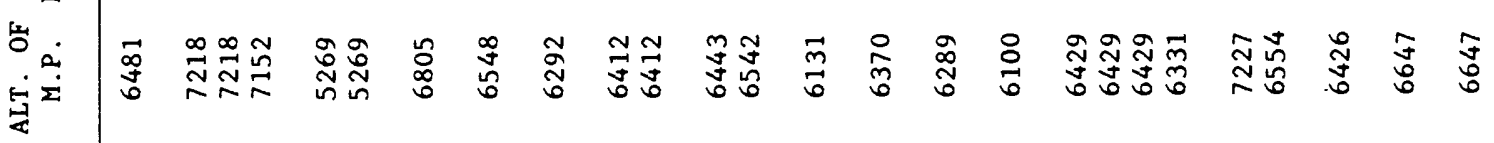

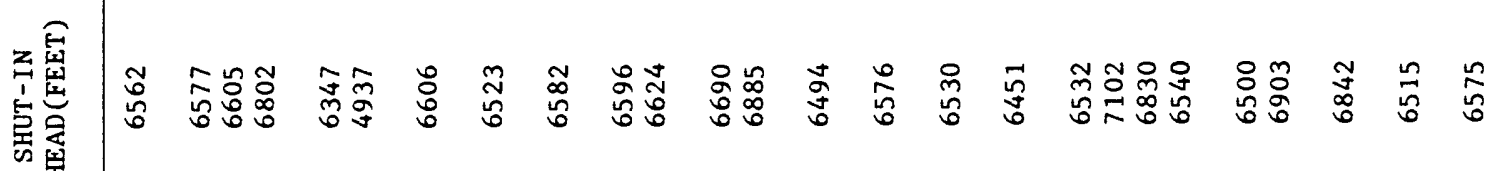

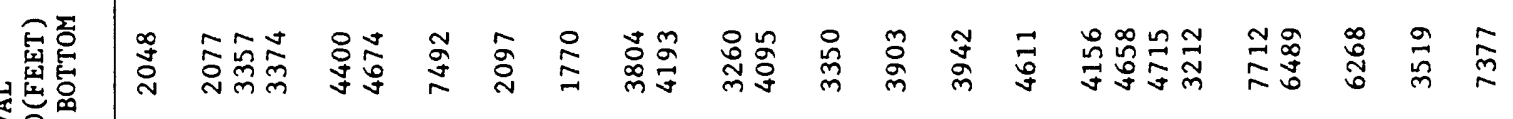

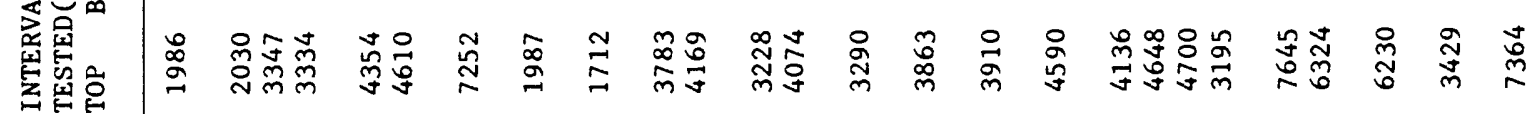

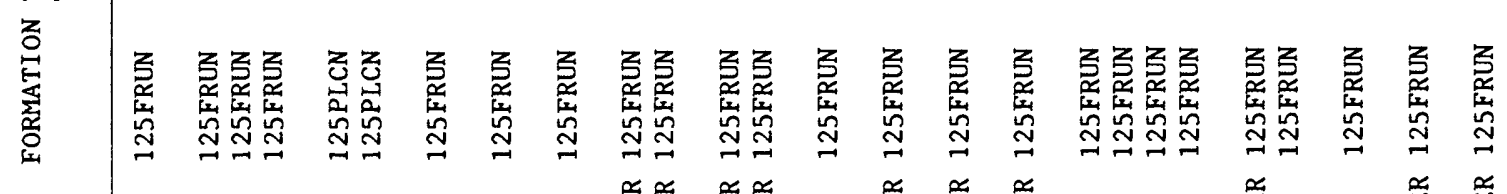

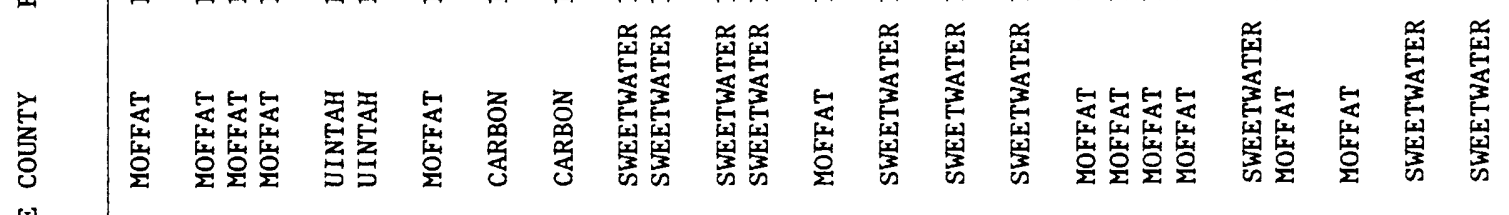

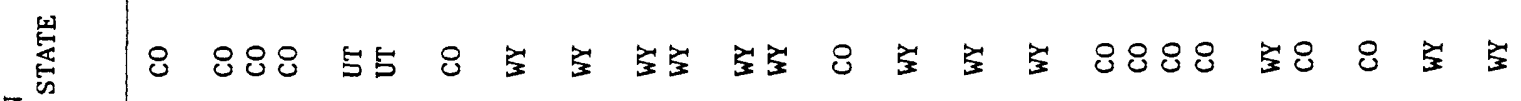

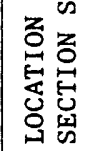

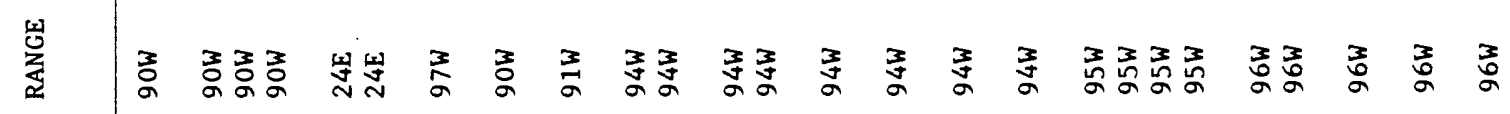

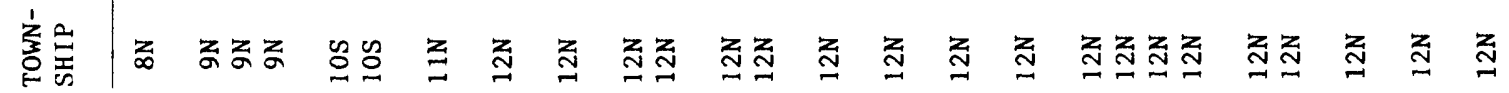




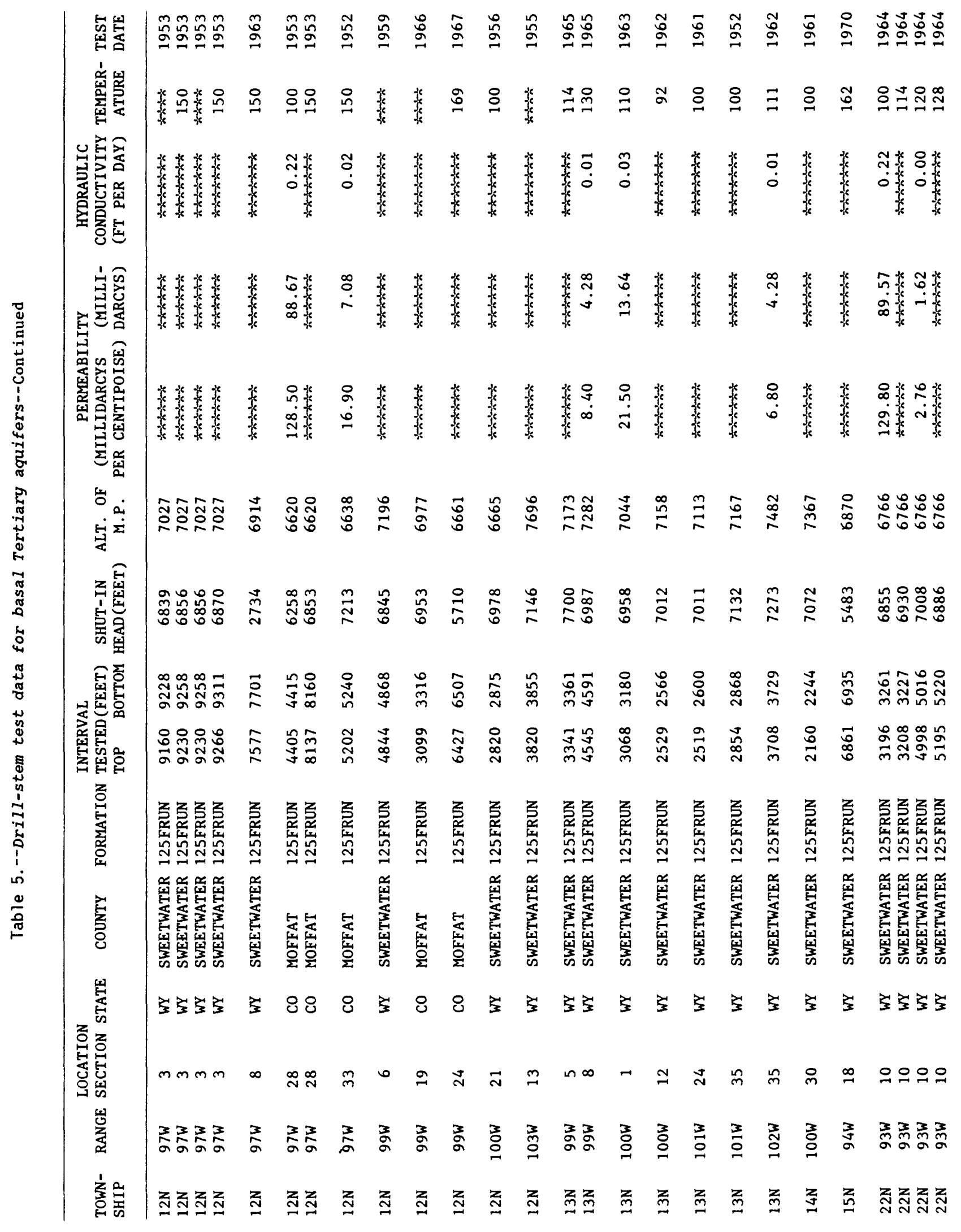




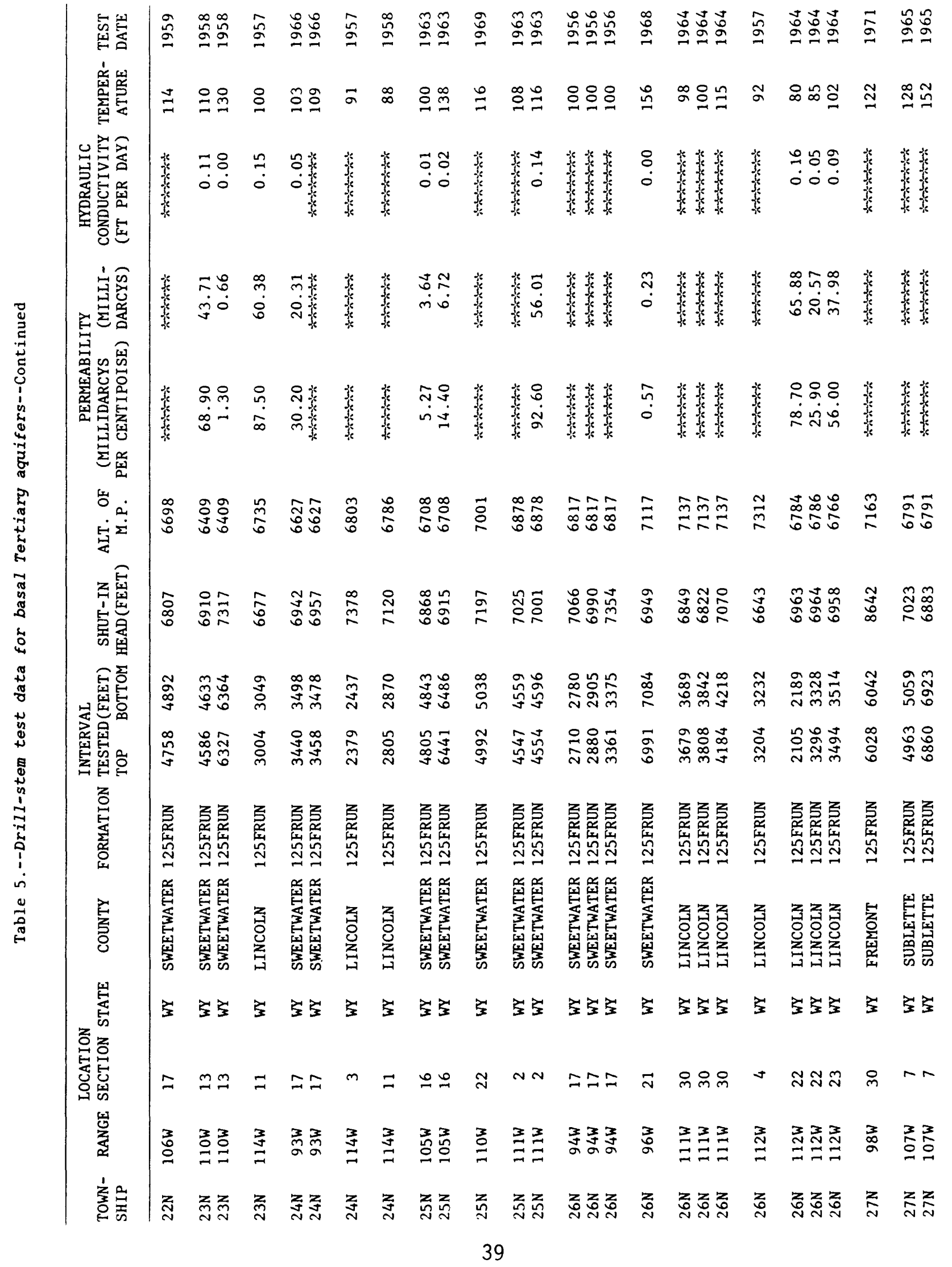




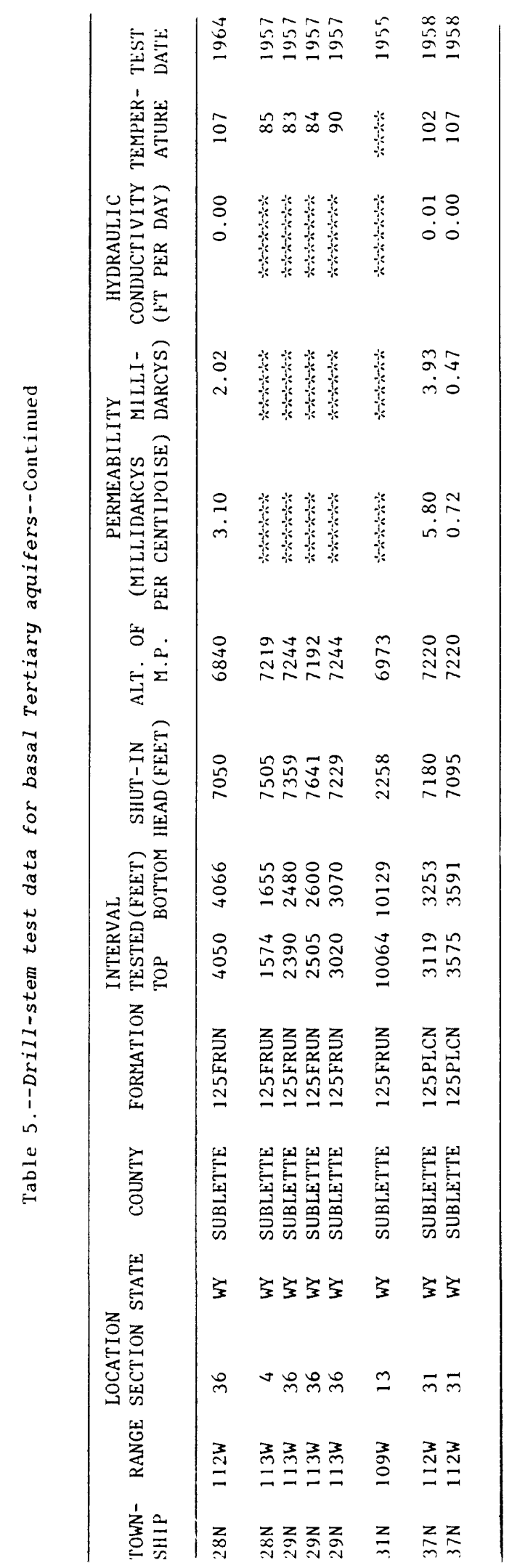




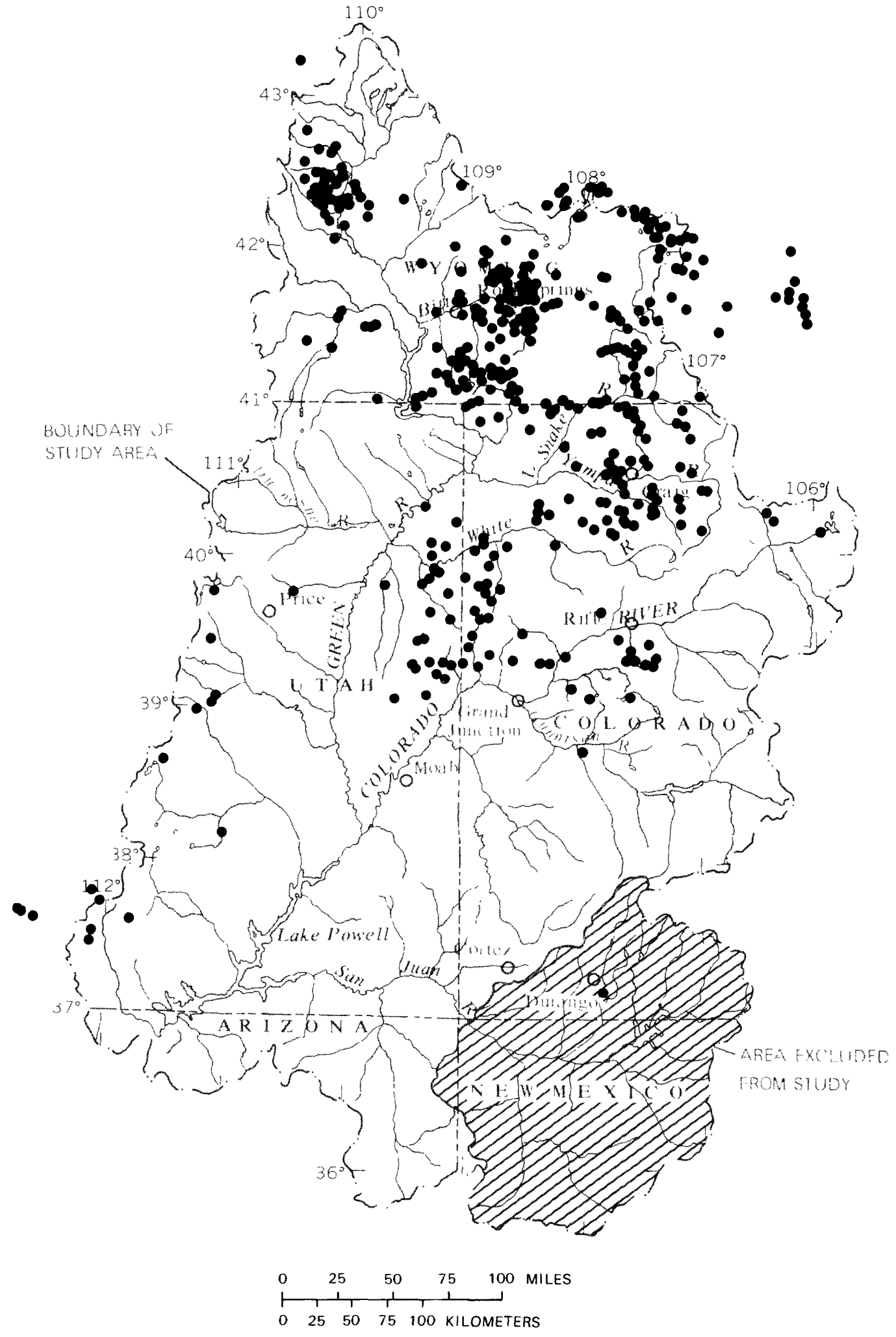

Figure 10.--Location of dri11-stem test data for upper Mesozoic confining layers and aquifers. 


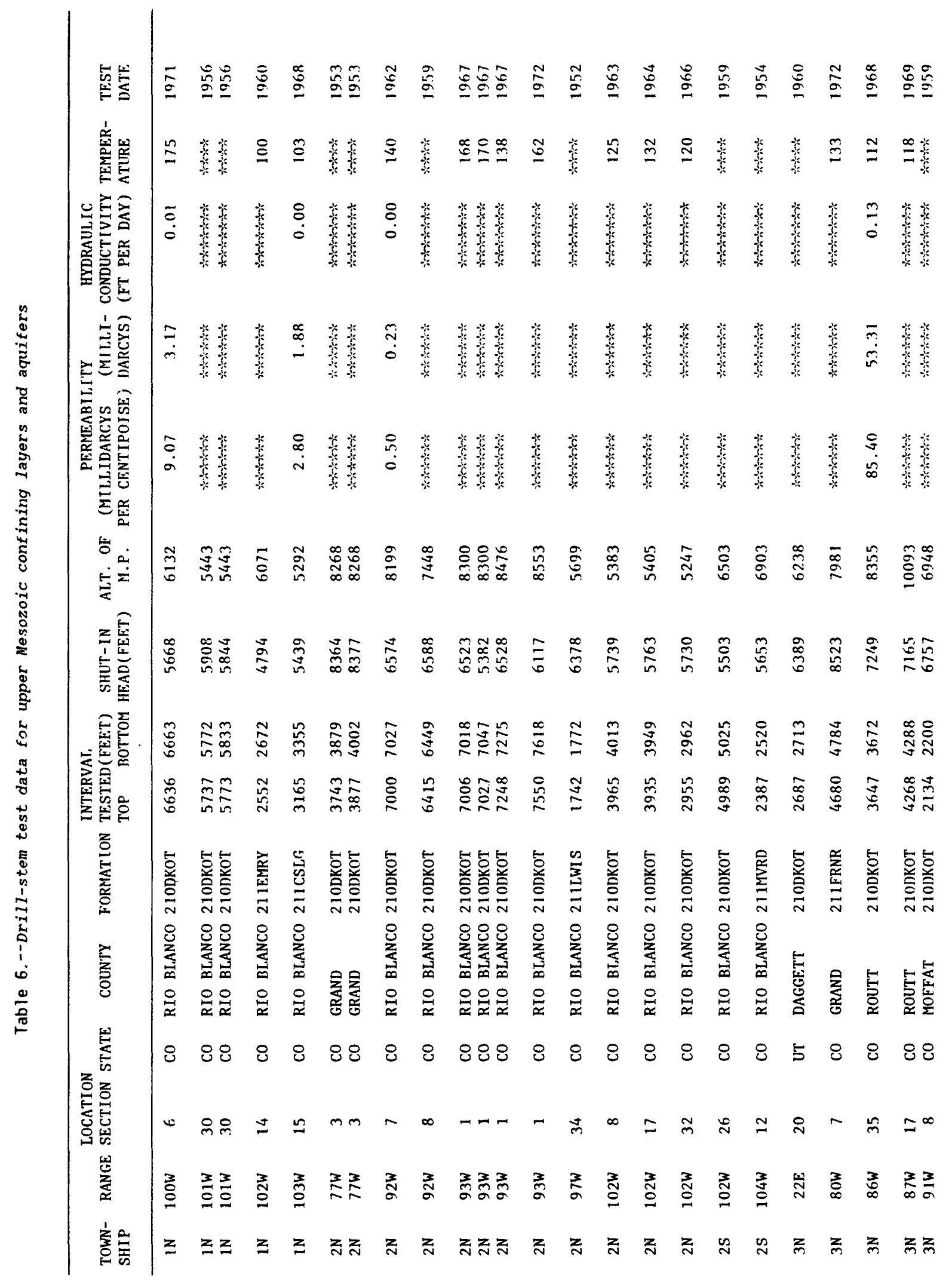




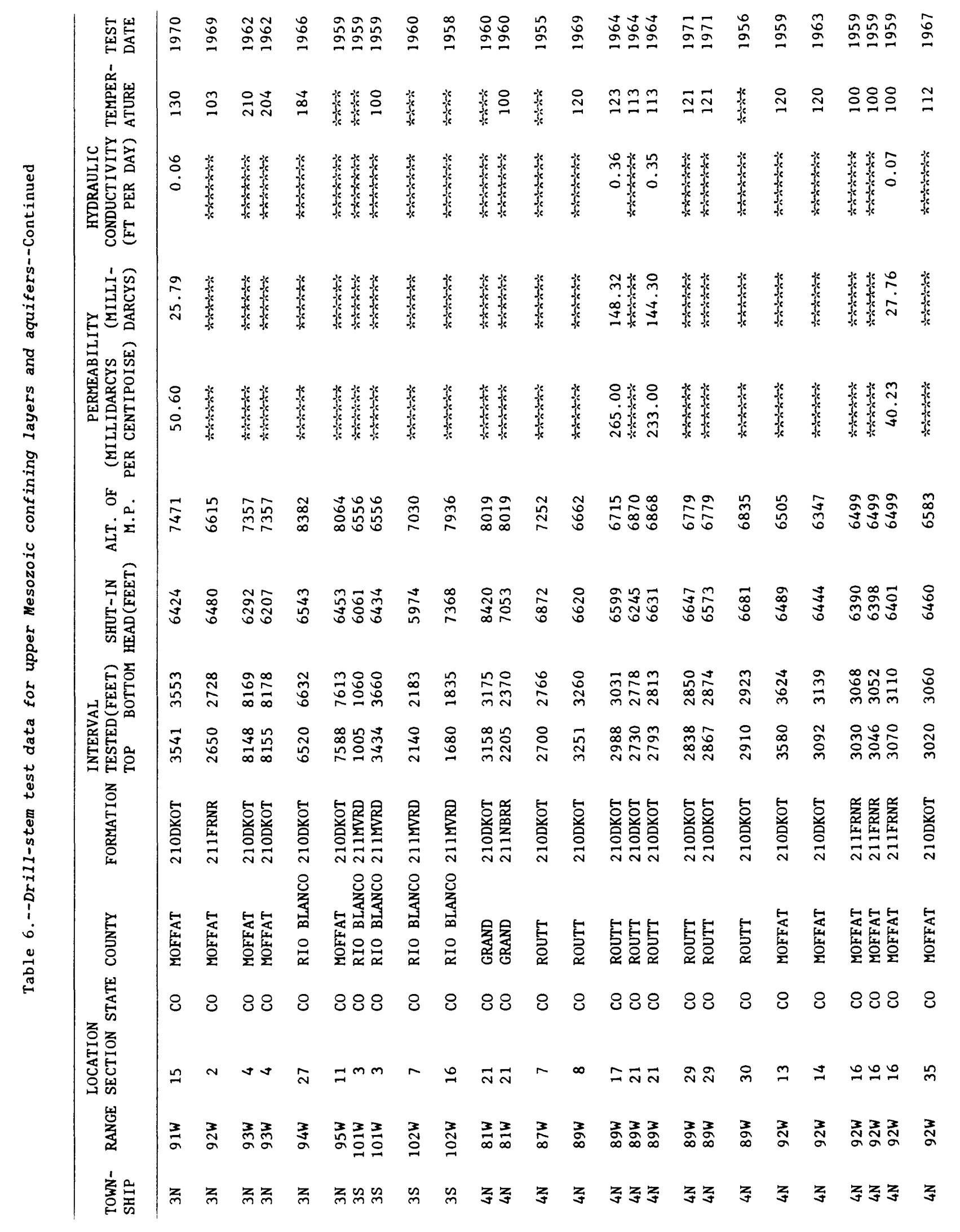




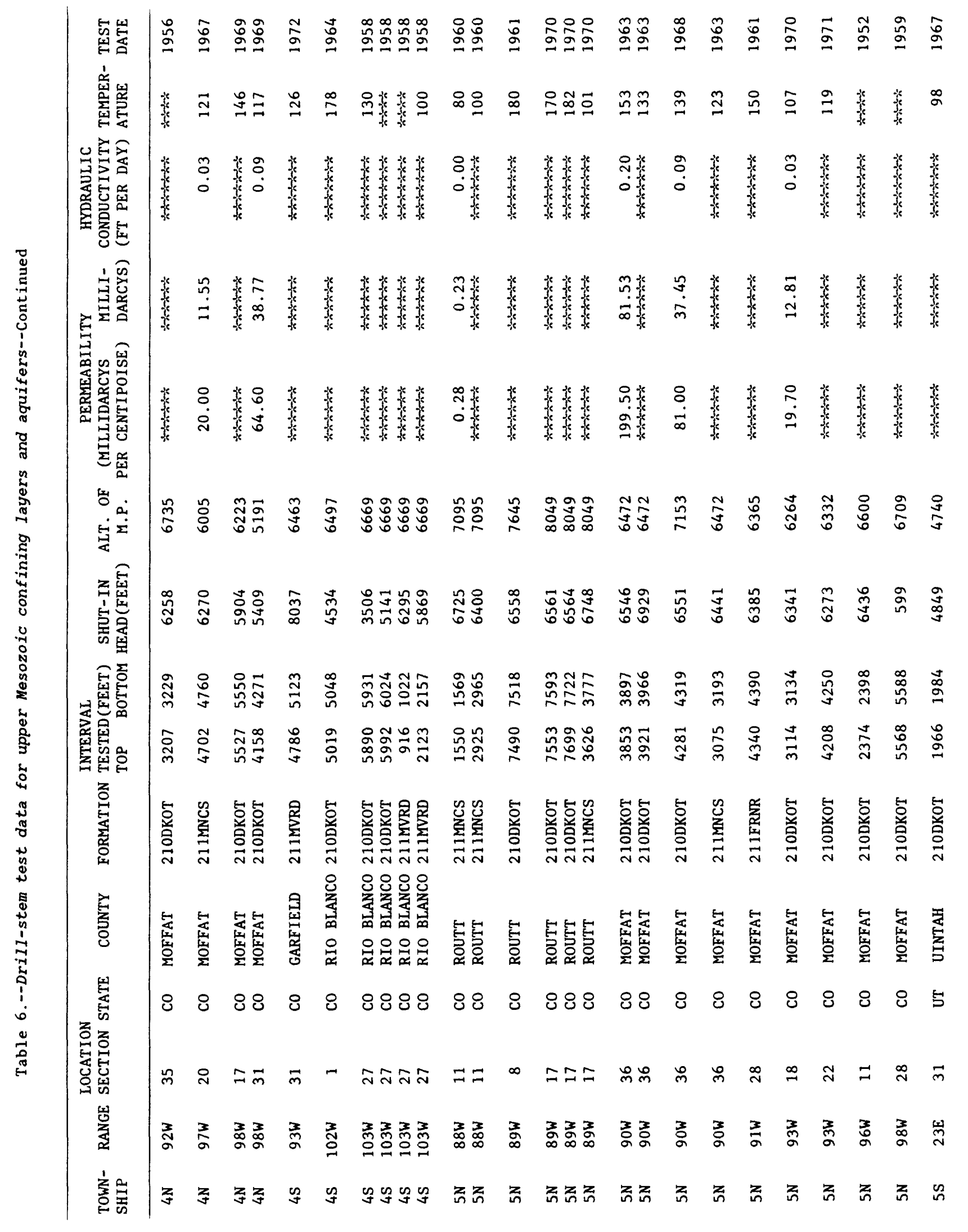




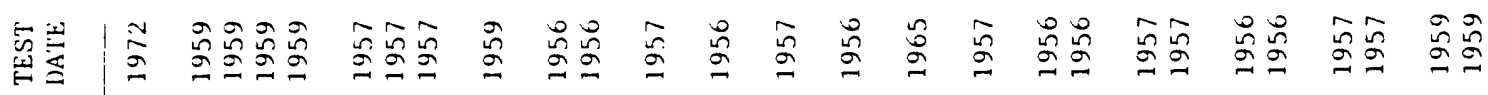

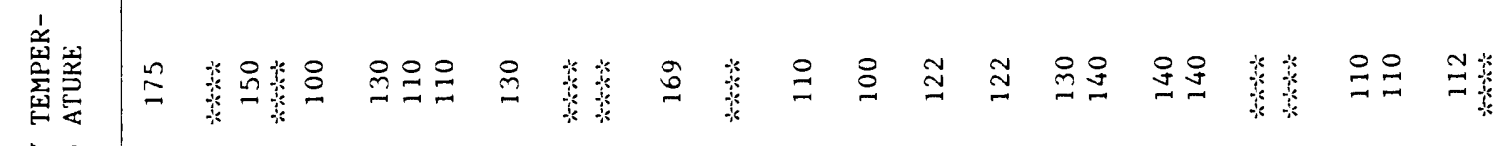

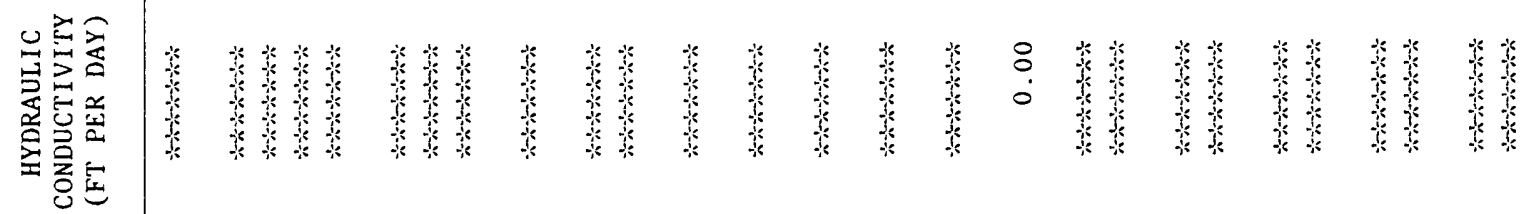

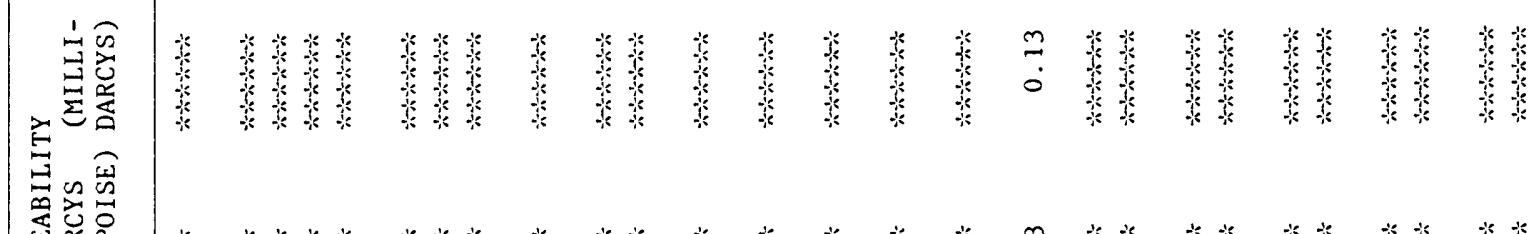

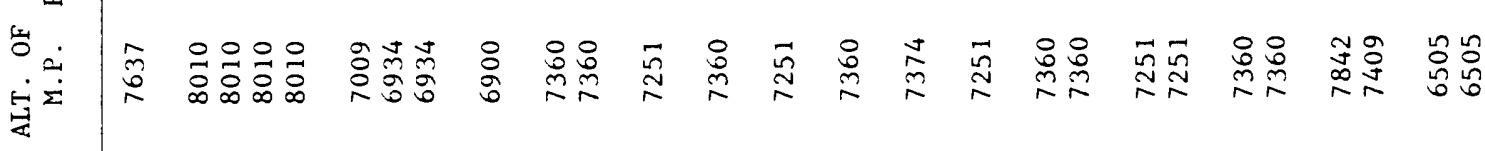

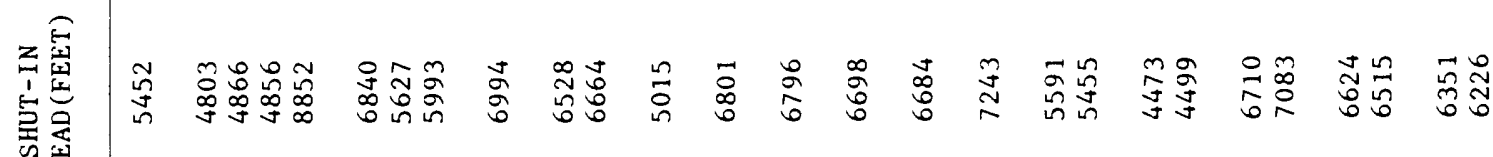

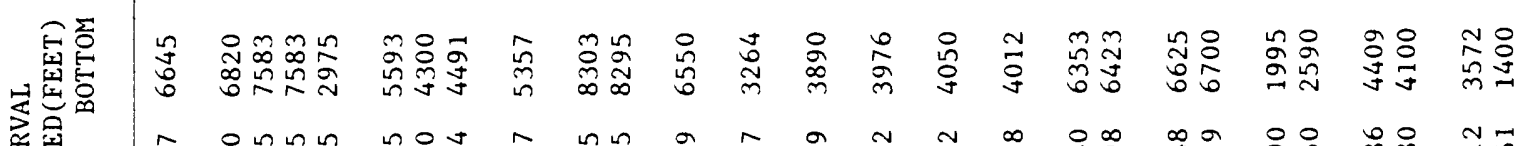

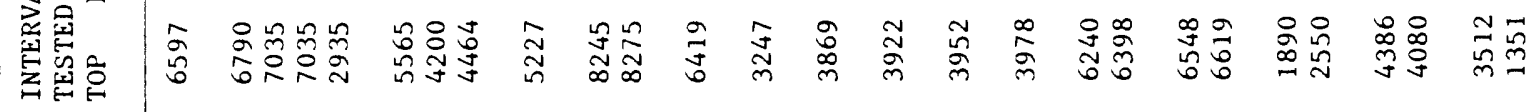

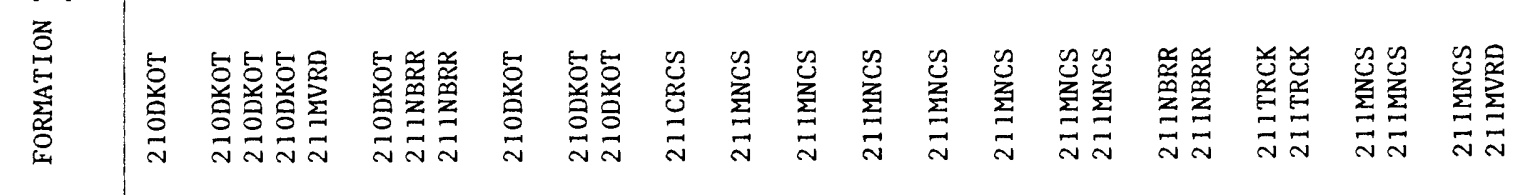

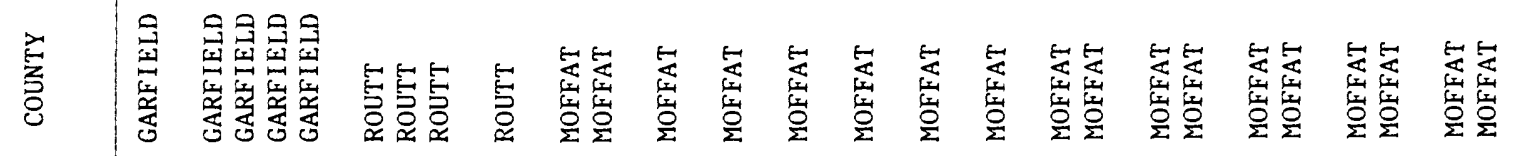
悹 88888888888888888888888888

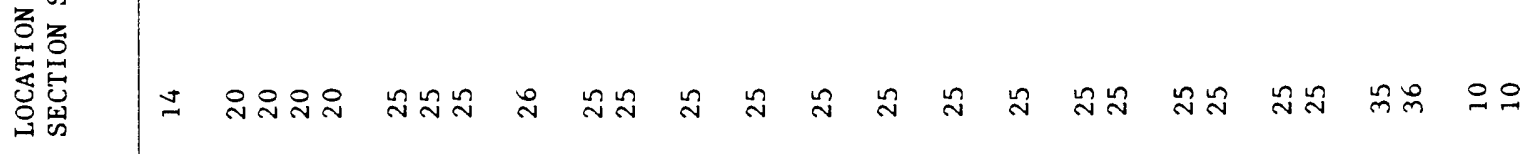

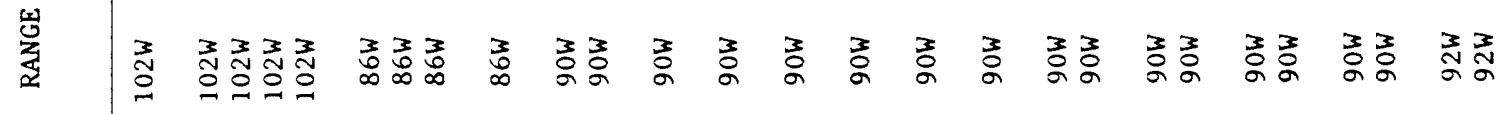

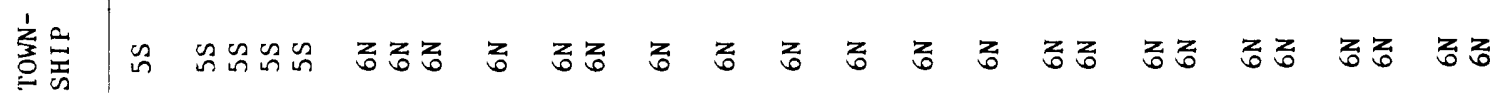




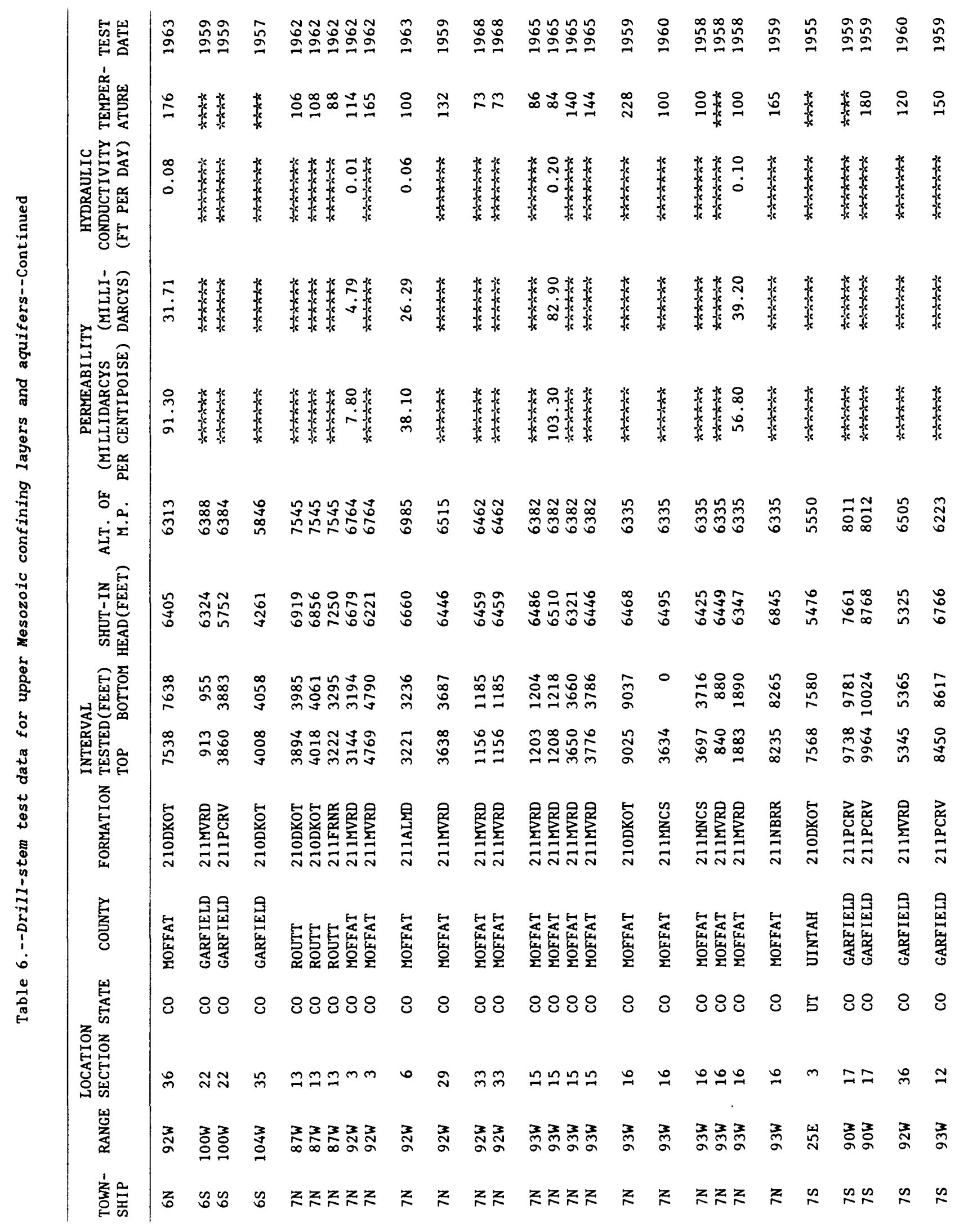




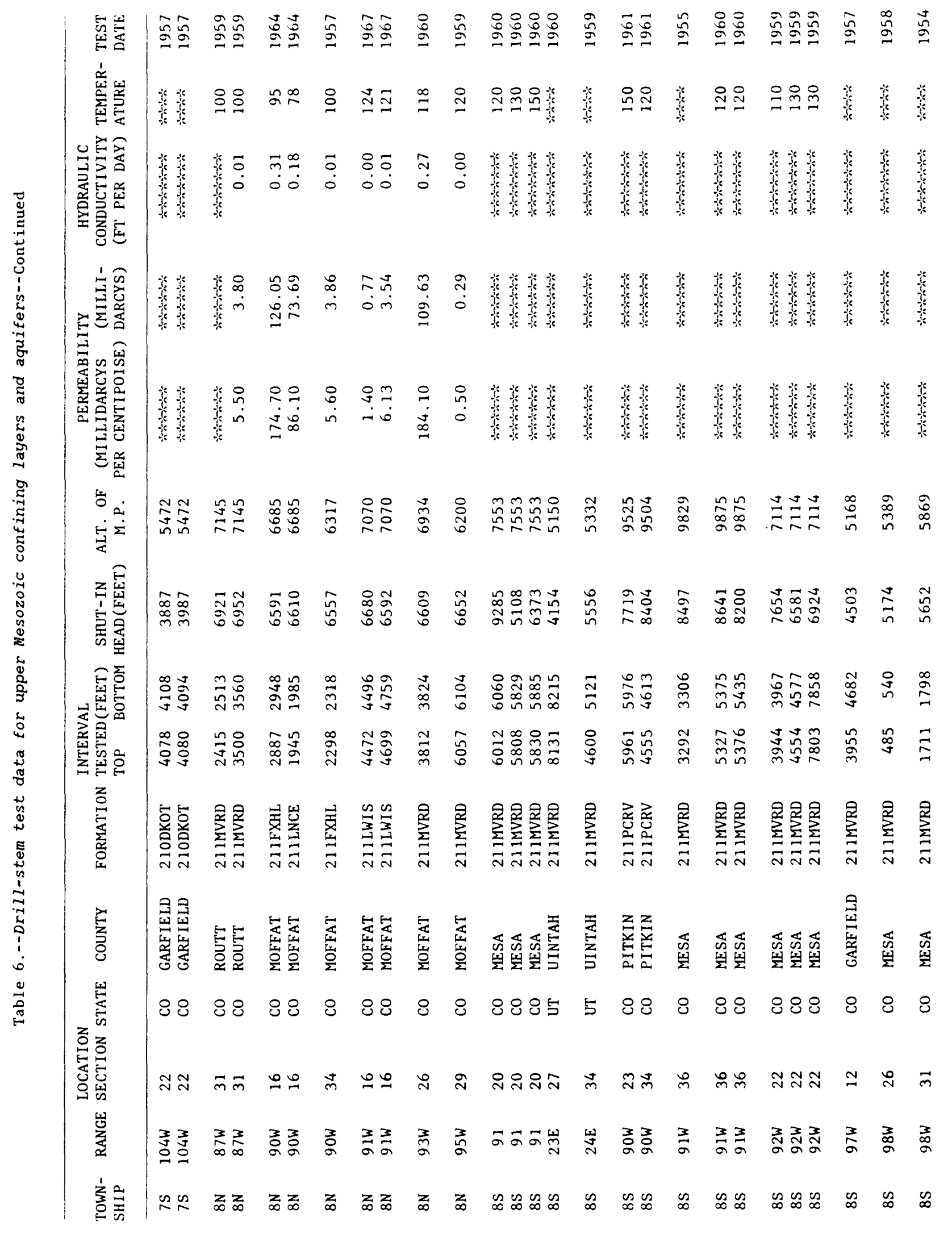




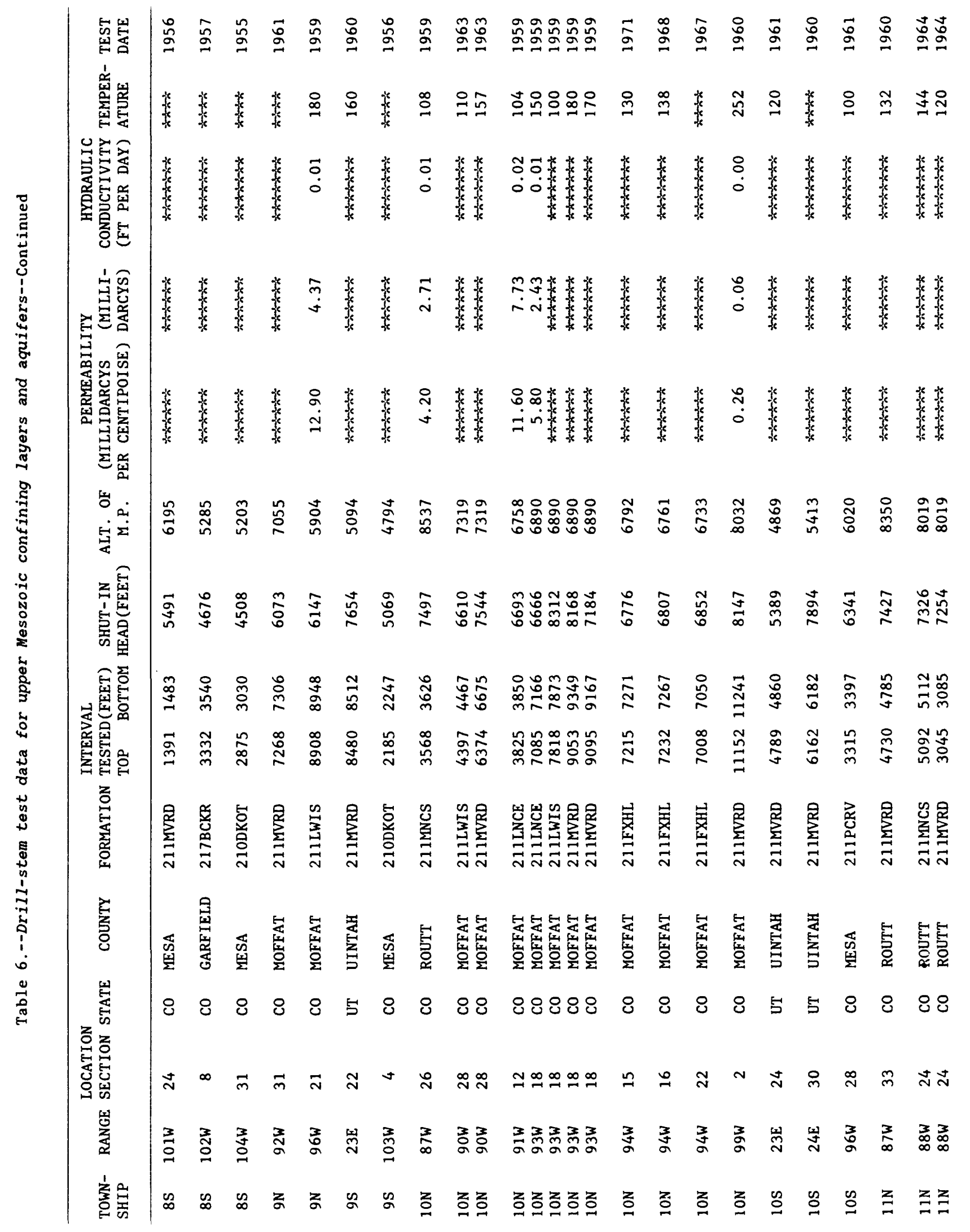




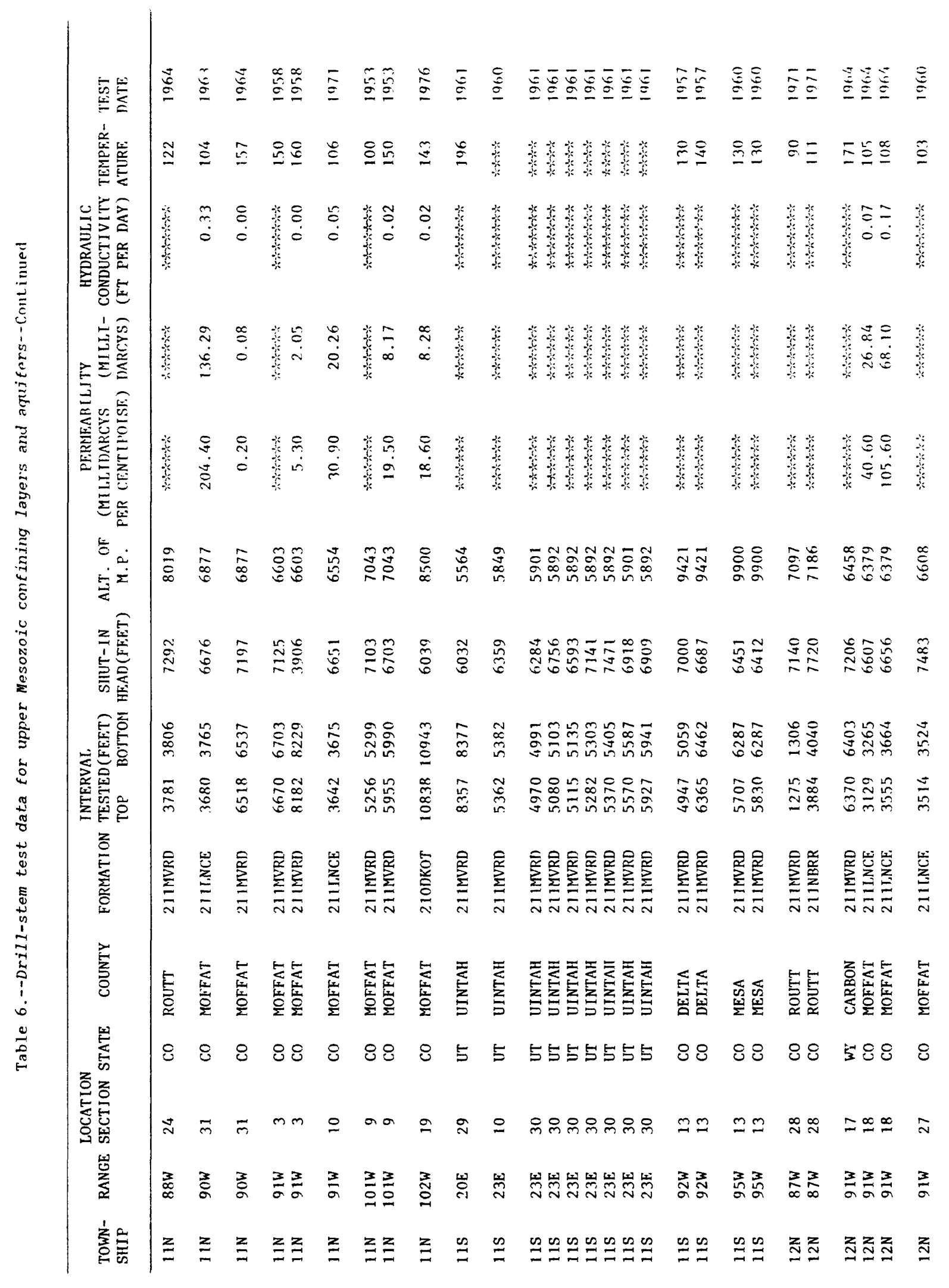




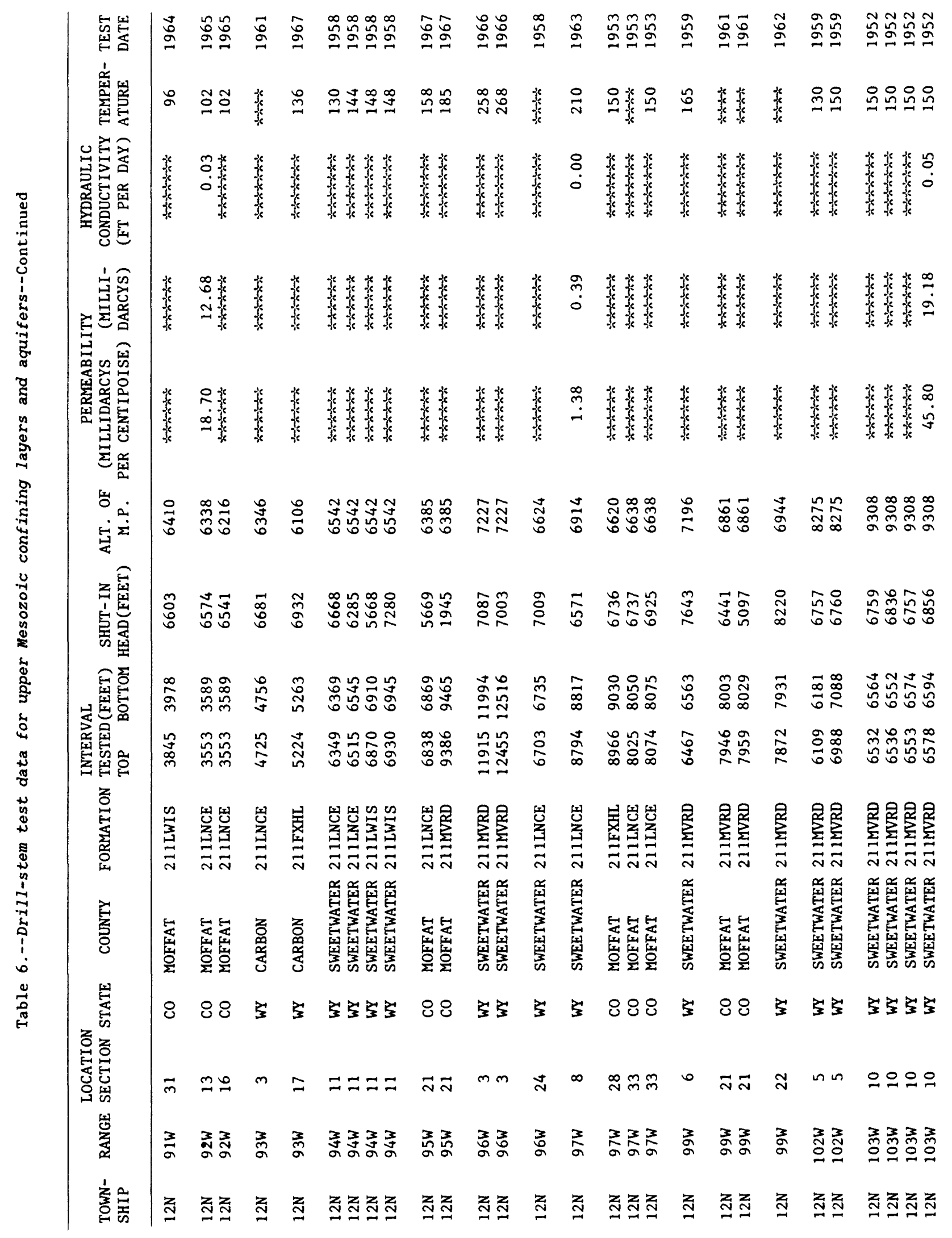




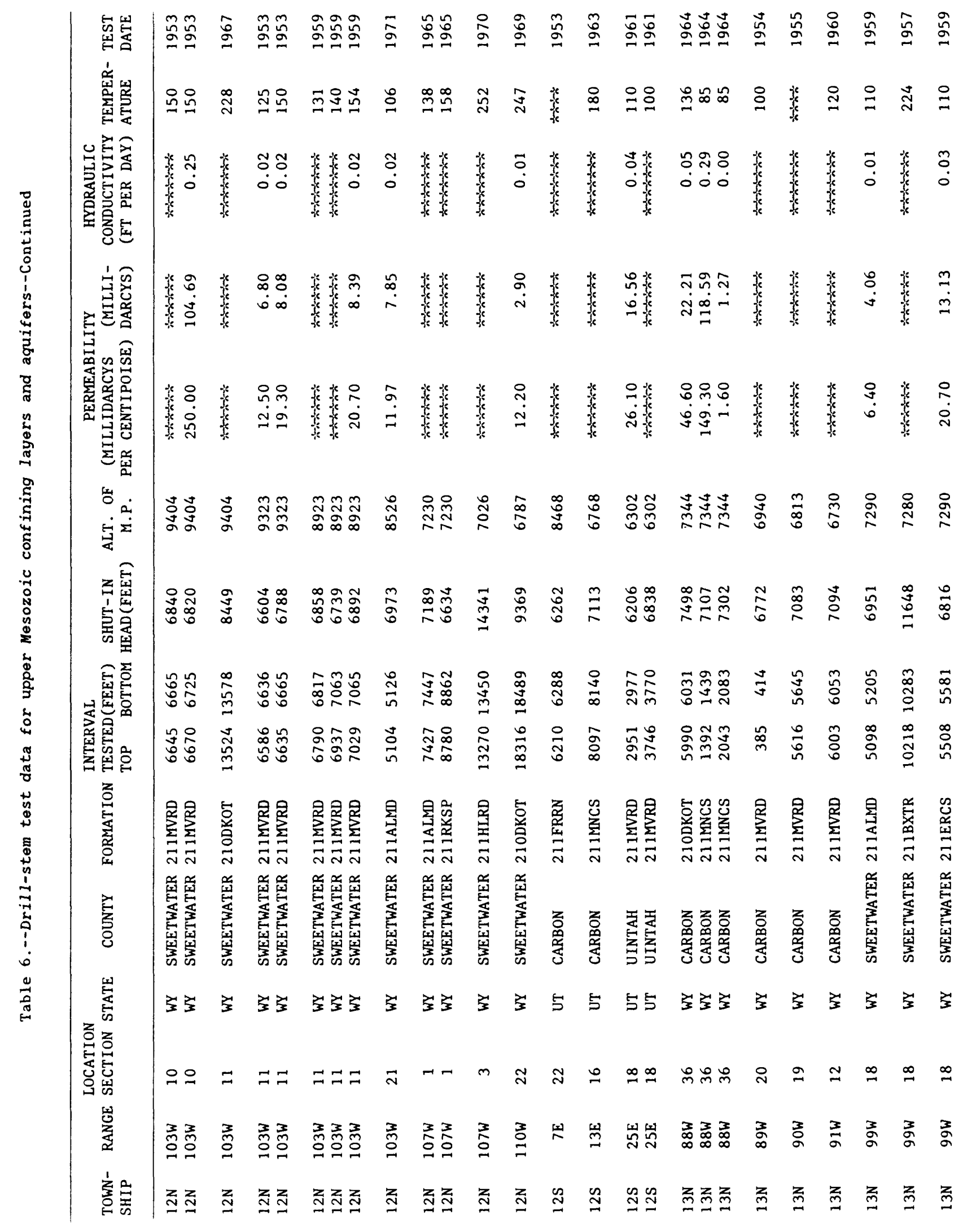




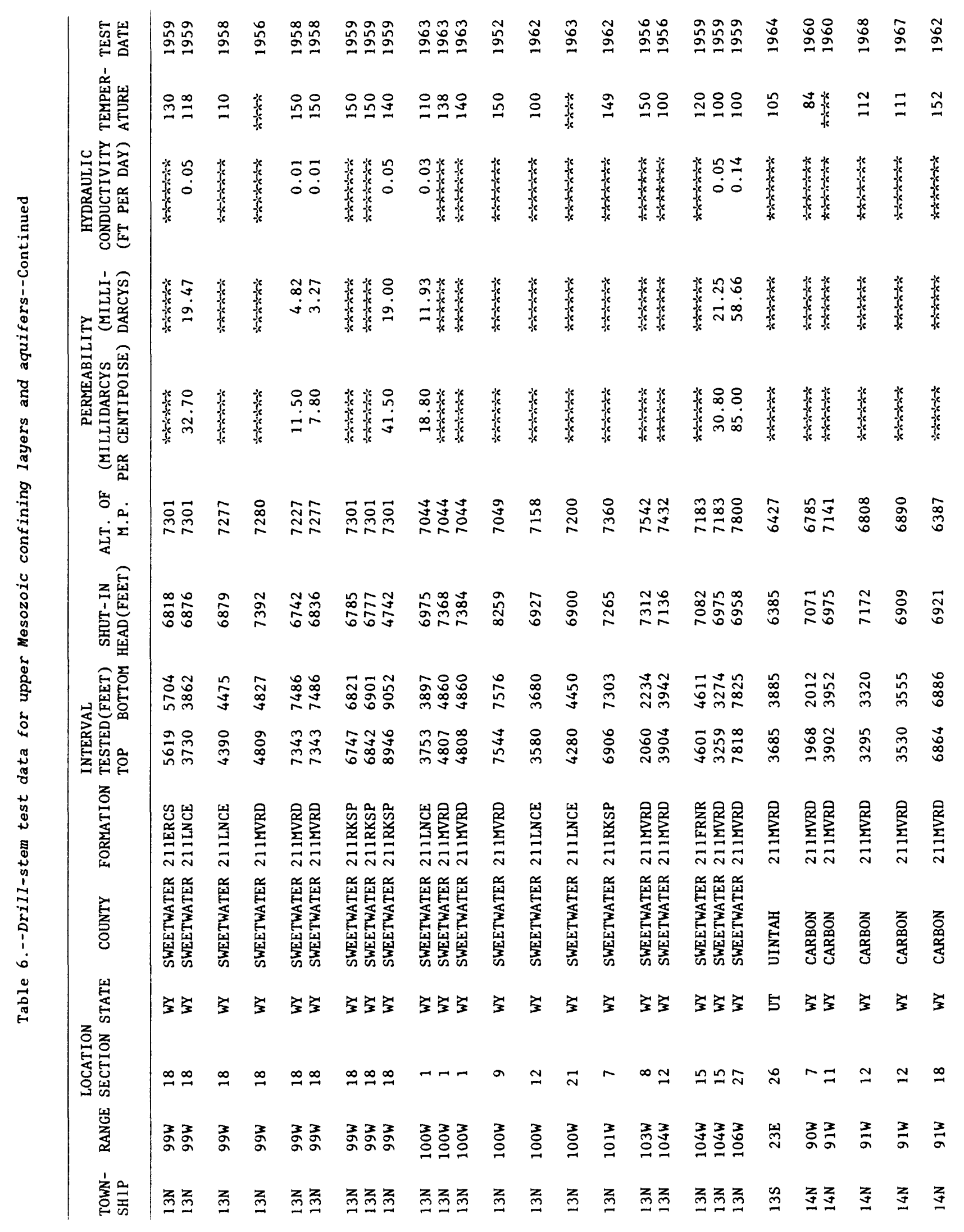




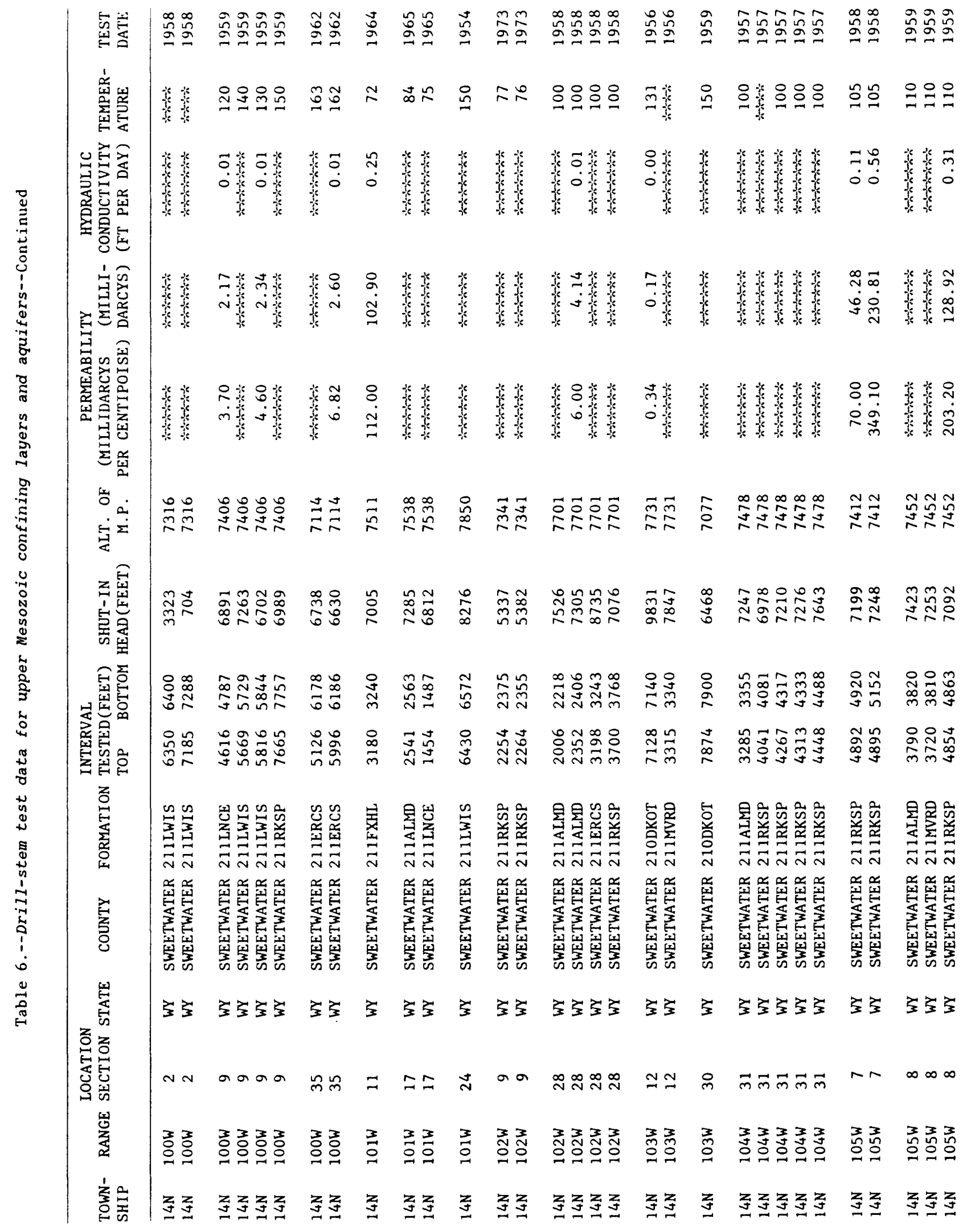




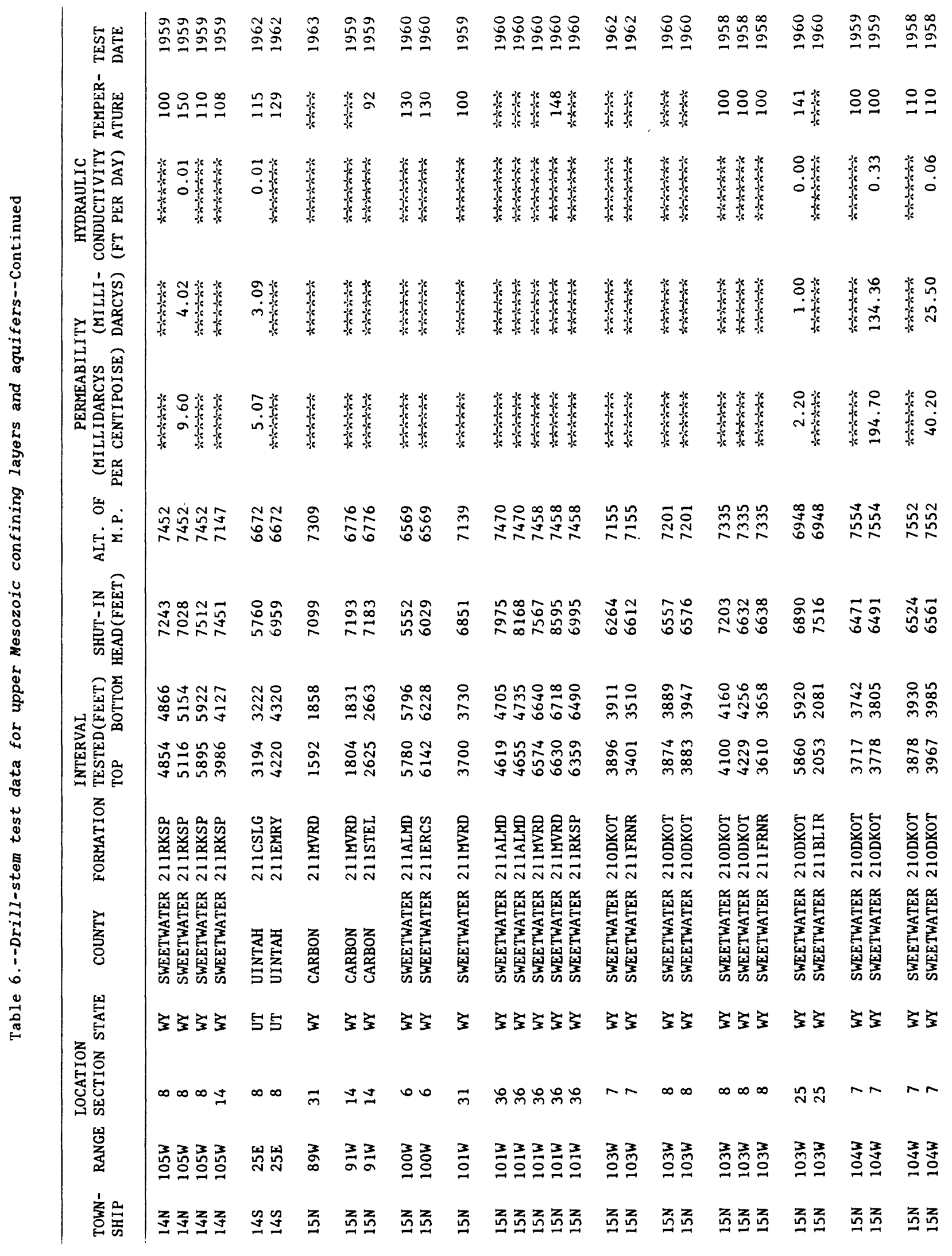




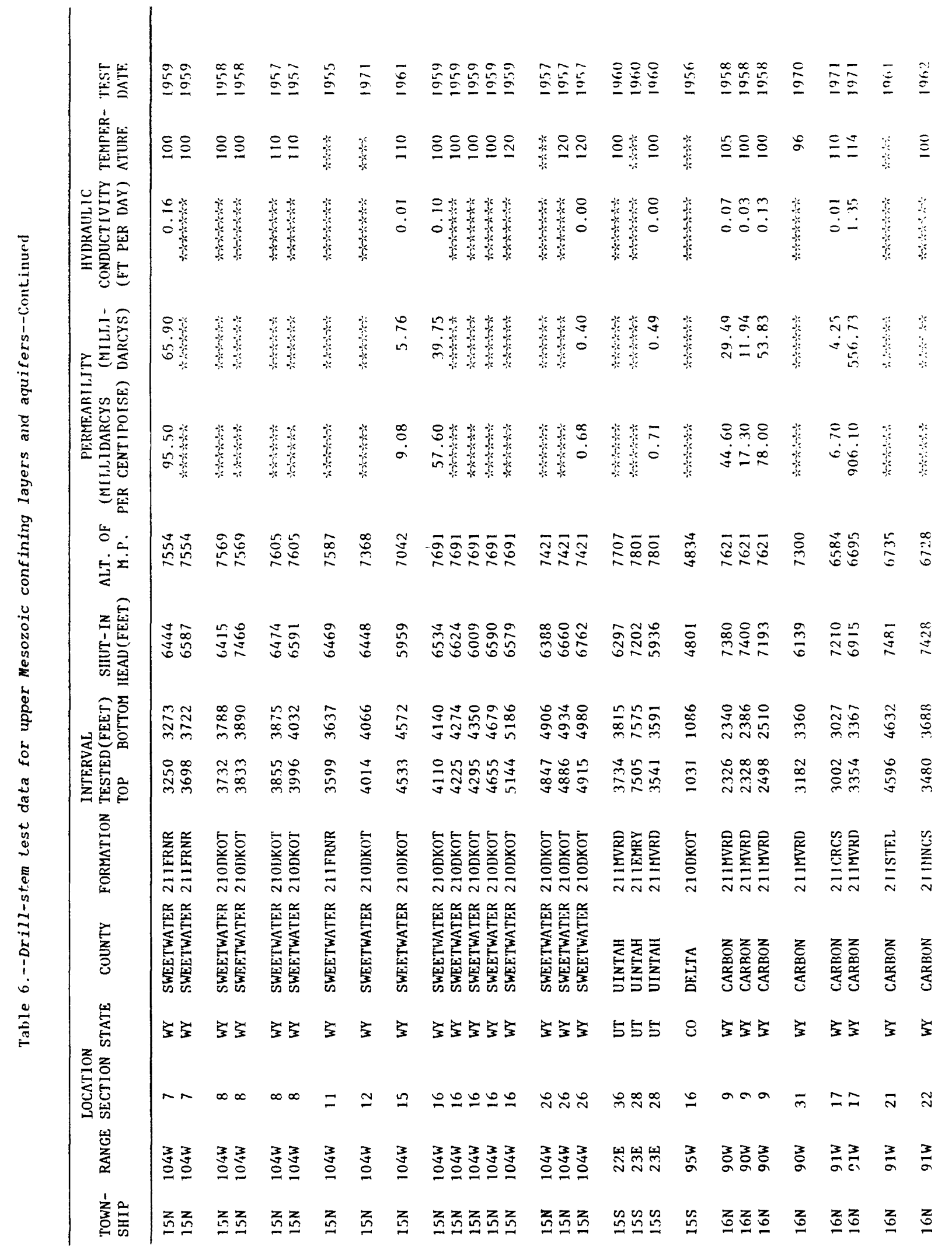




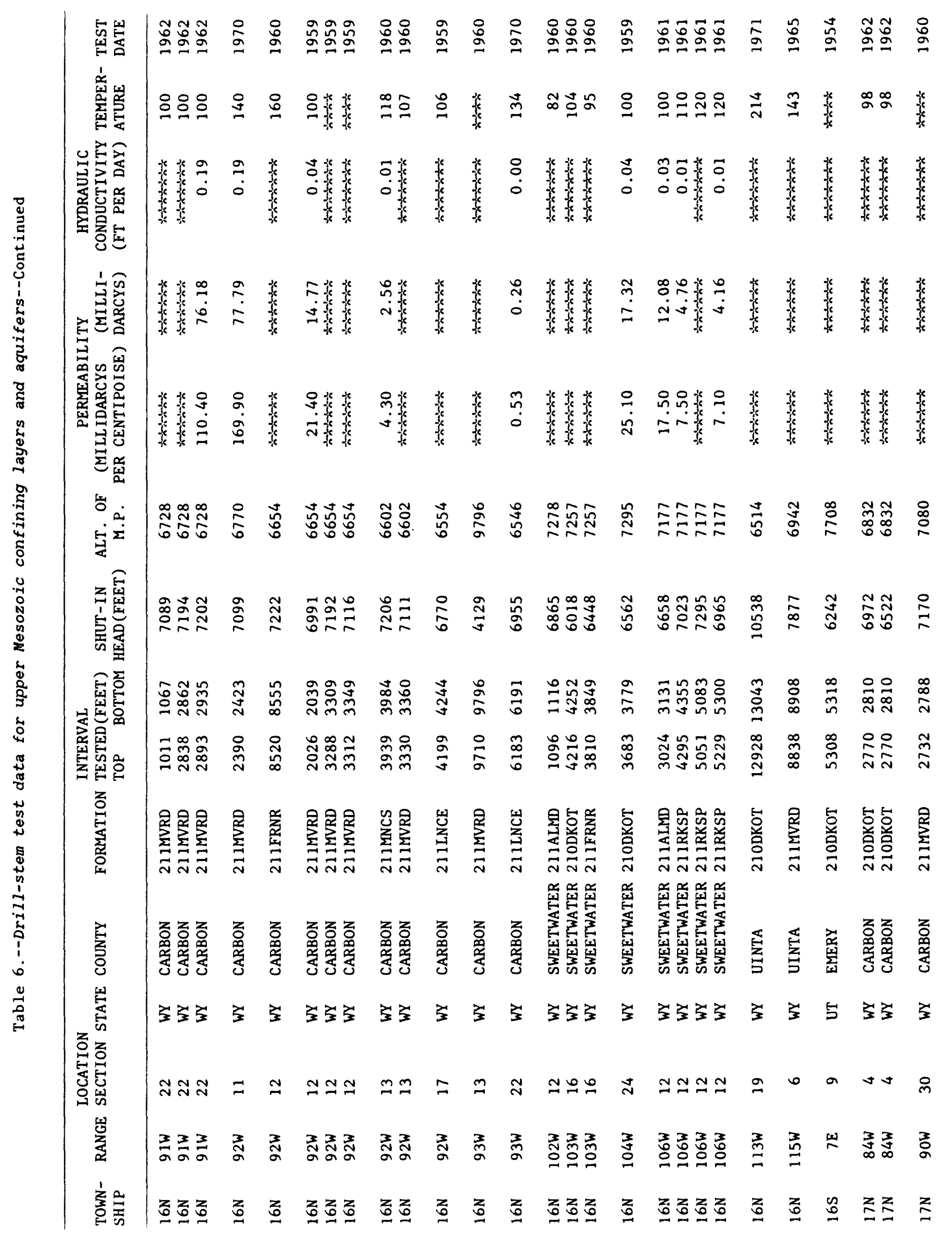




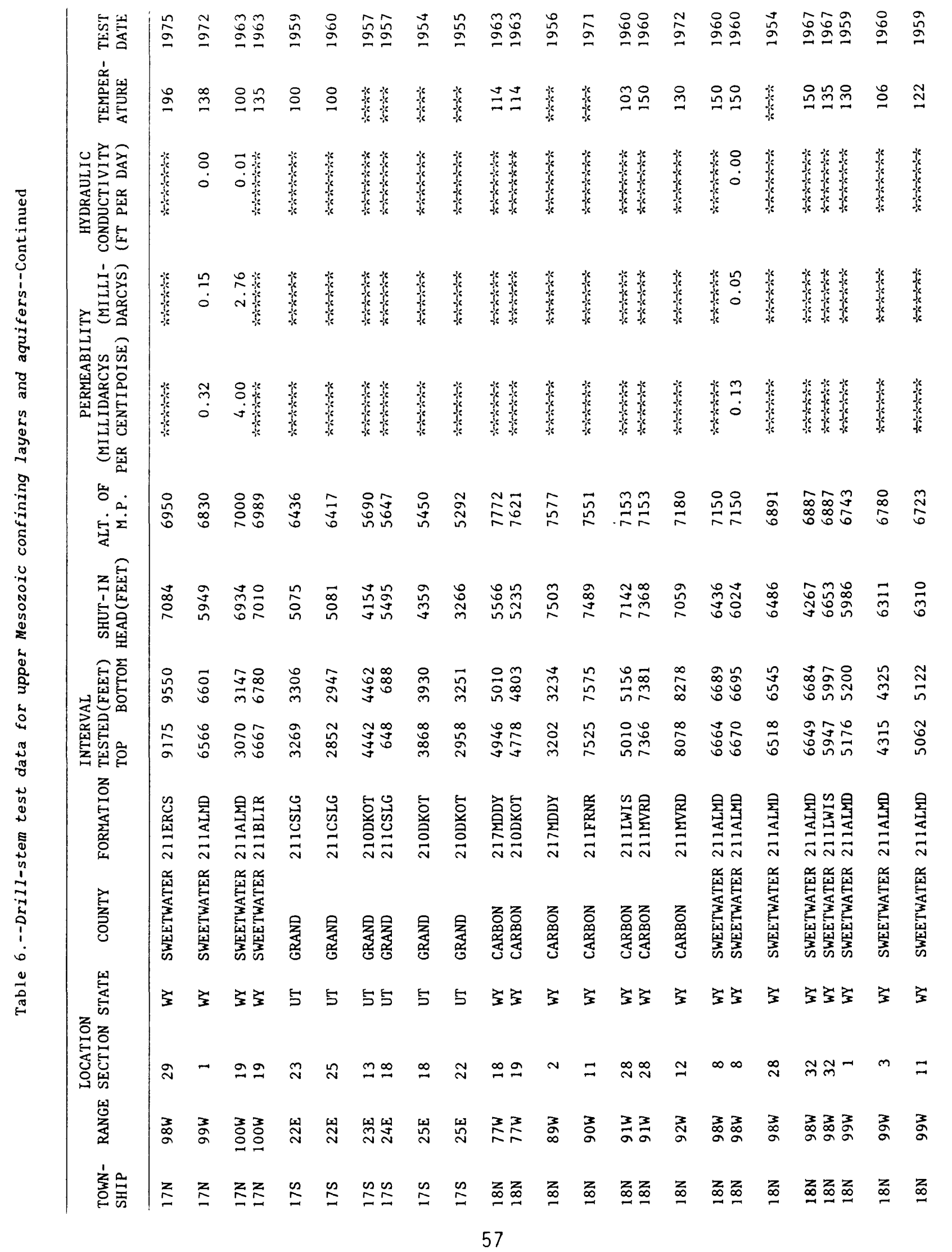




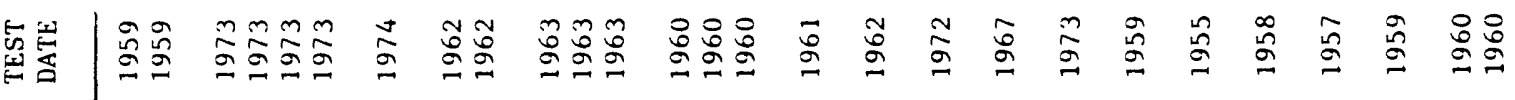

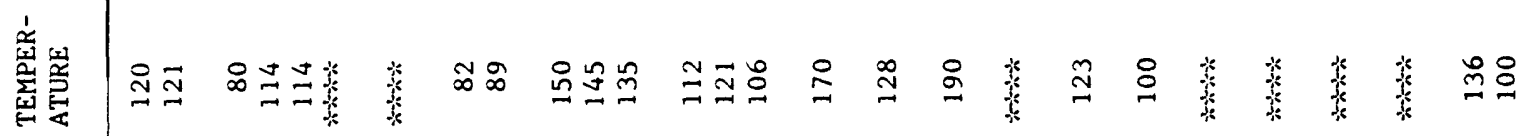

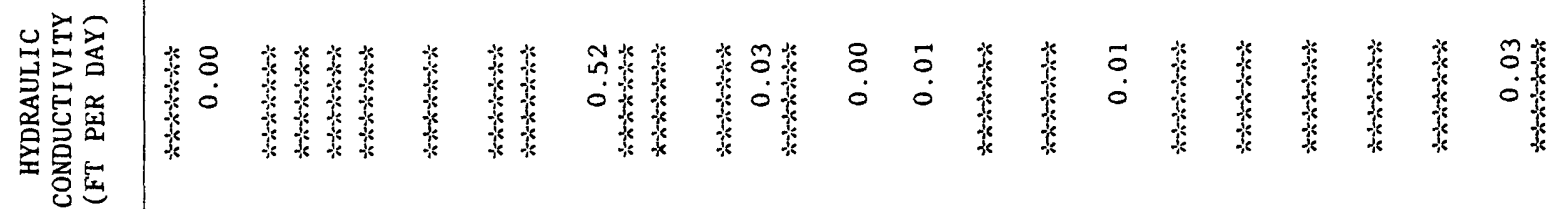
它爱 要递

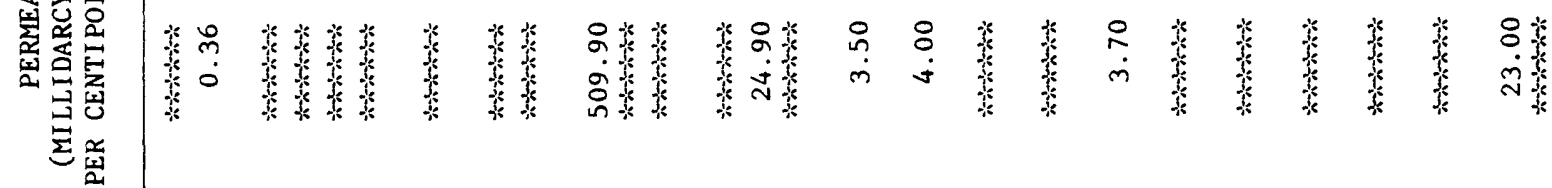

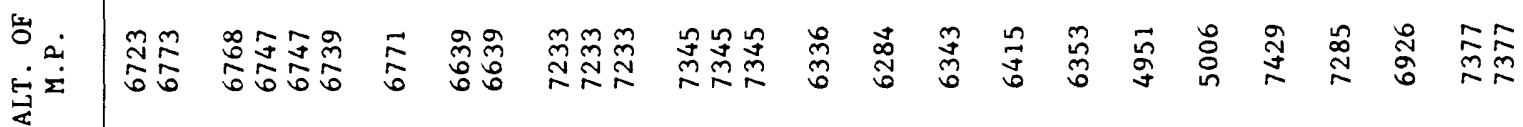
का

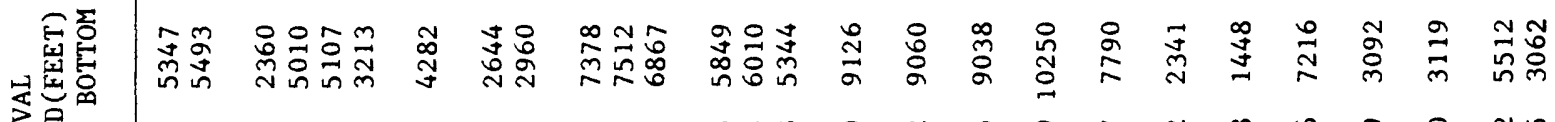
荡舫

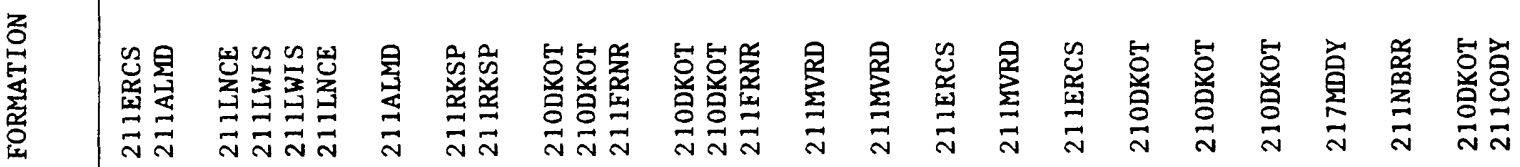

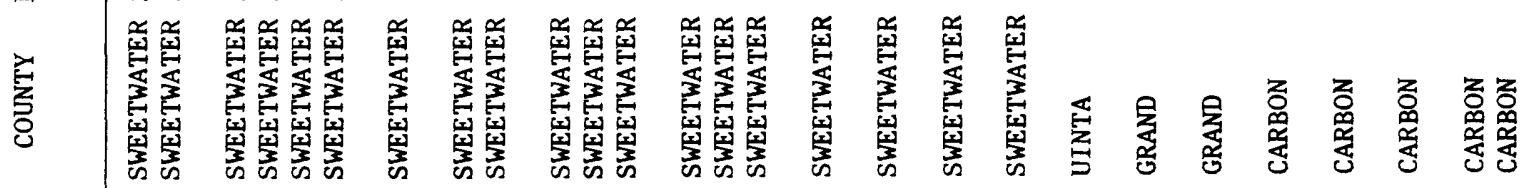
崖

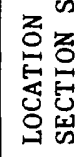

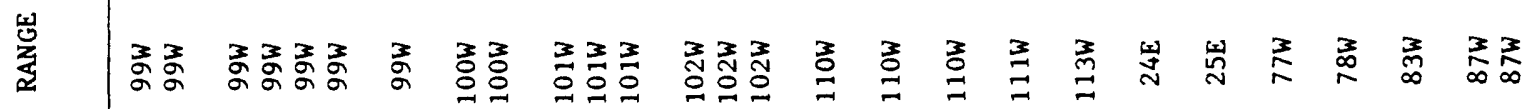

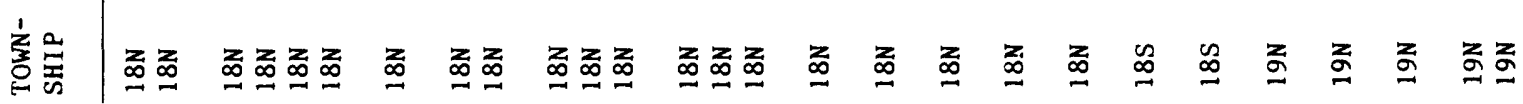




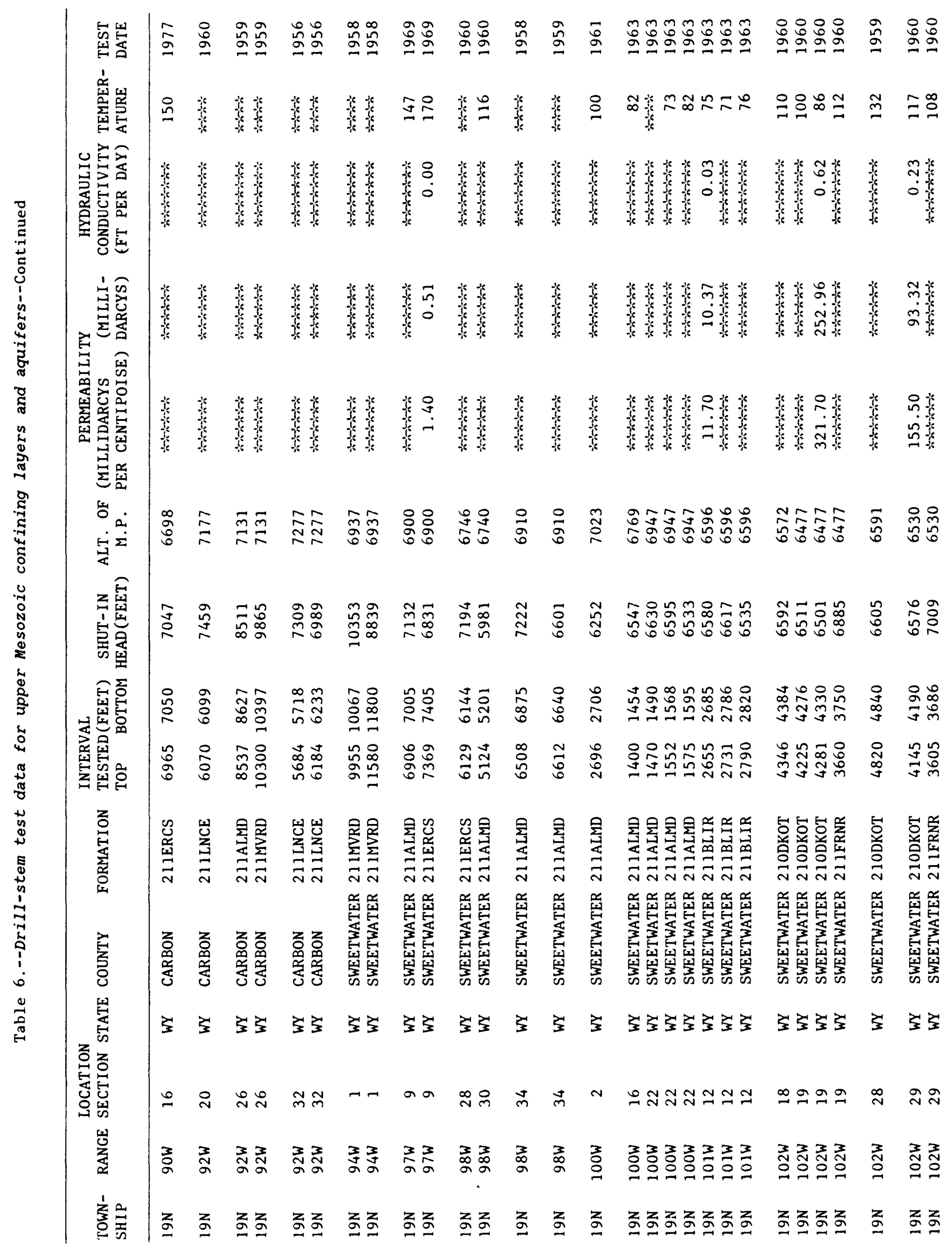




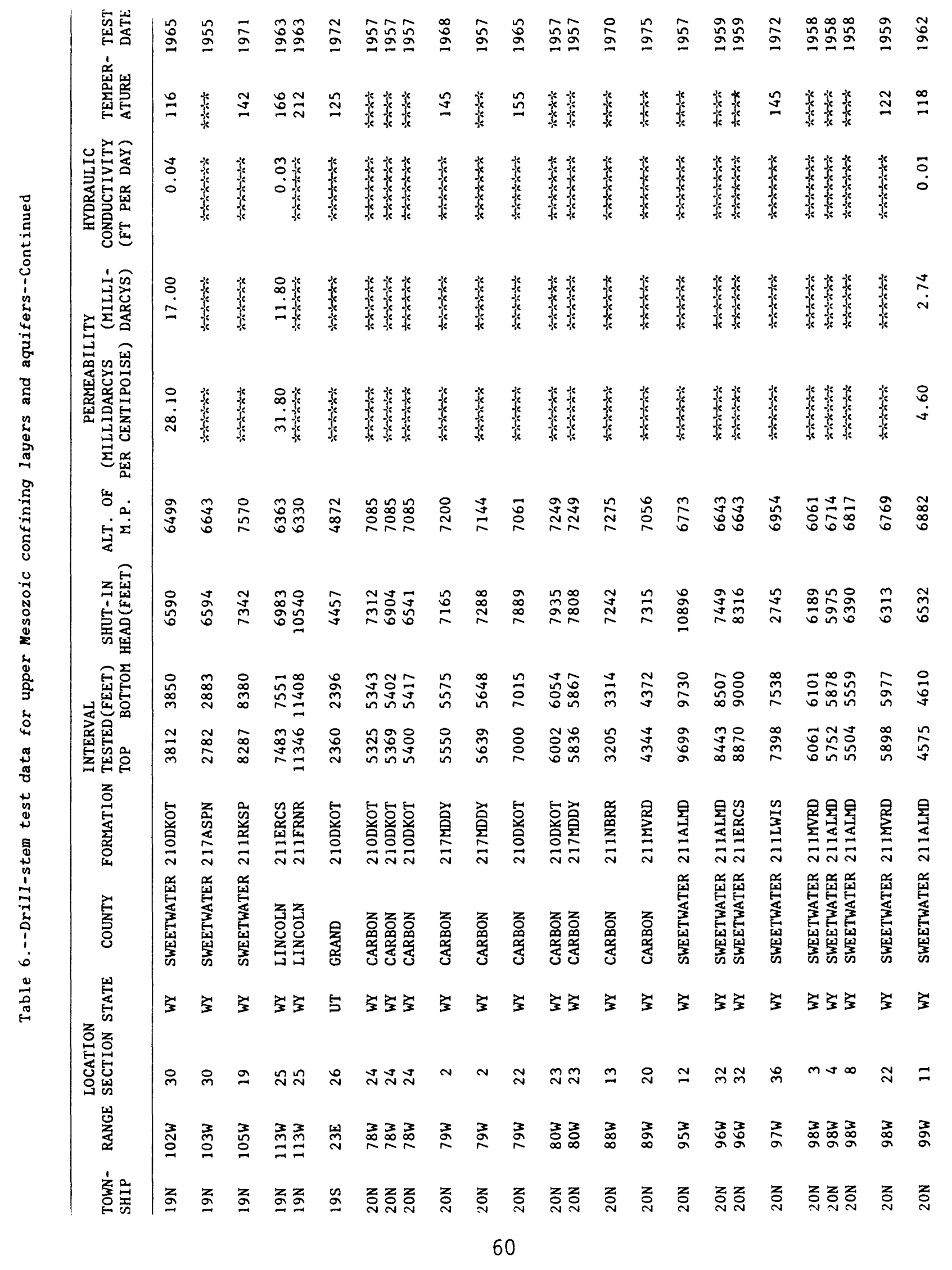




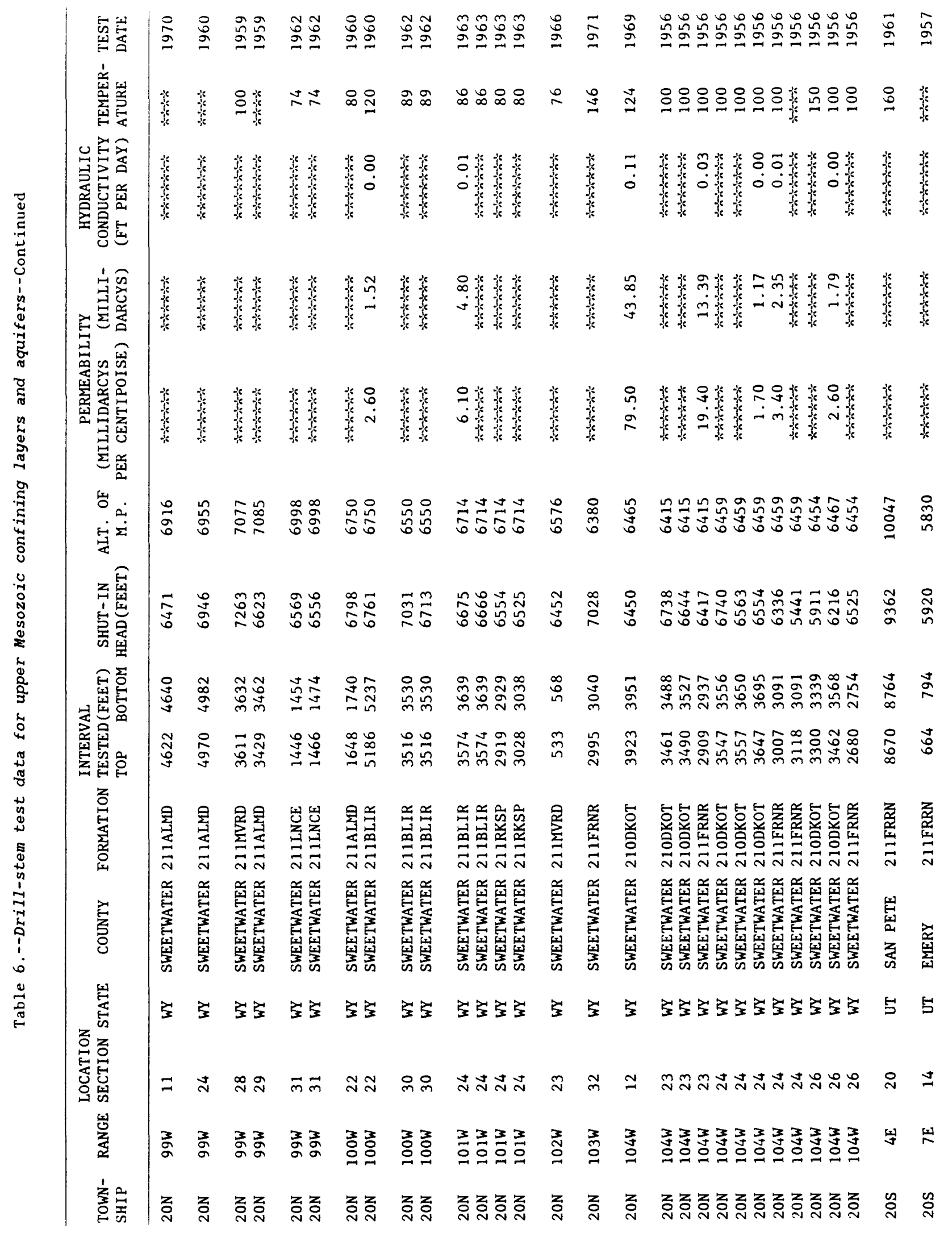




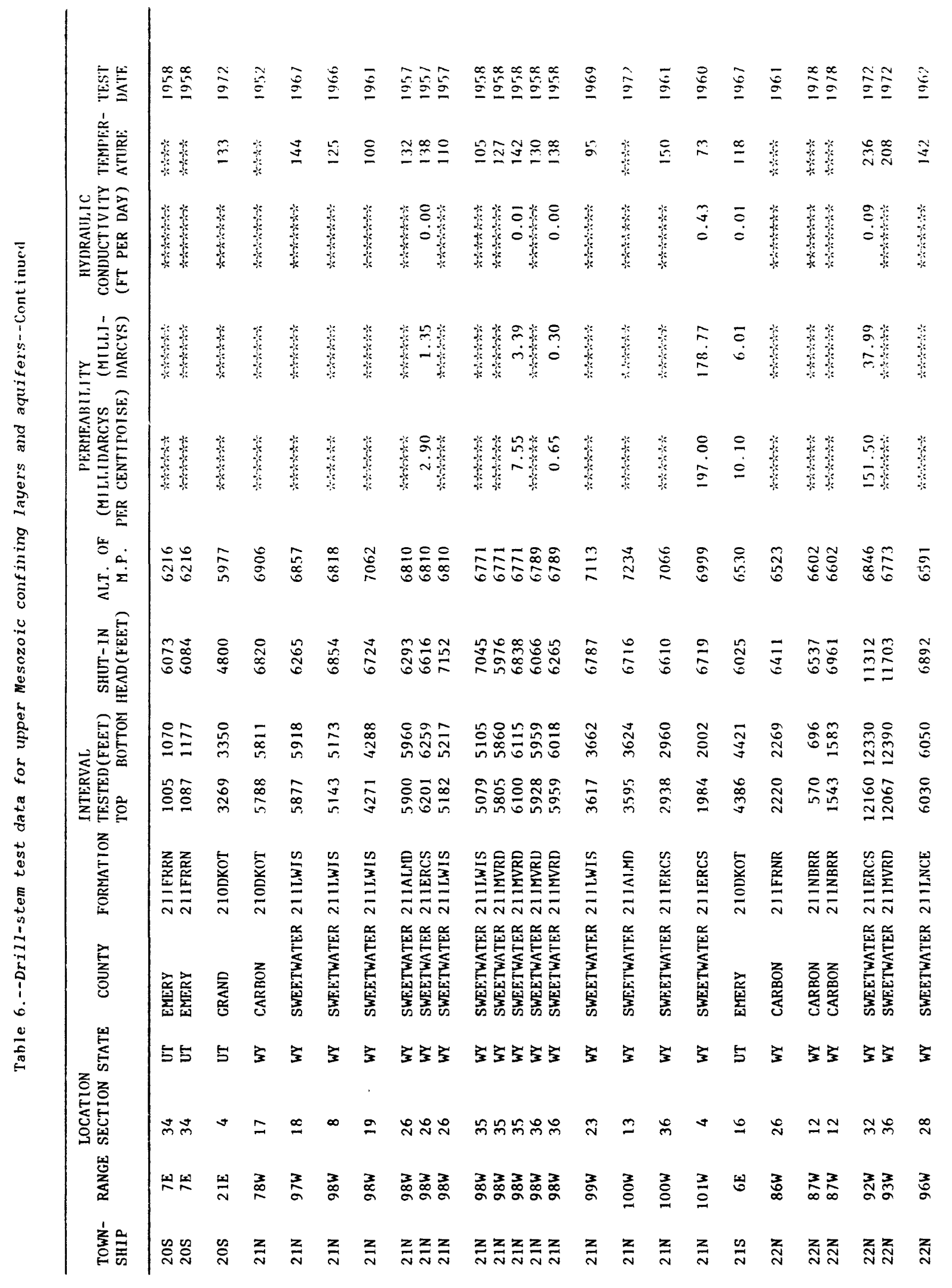




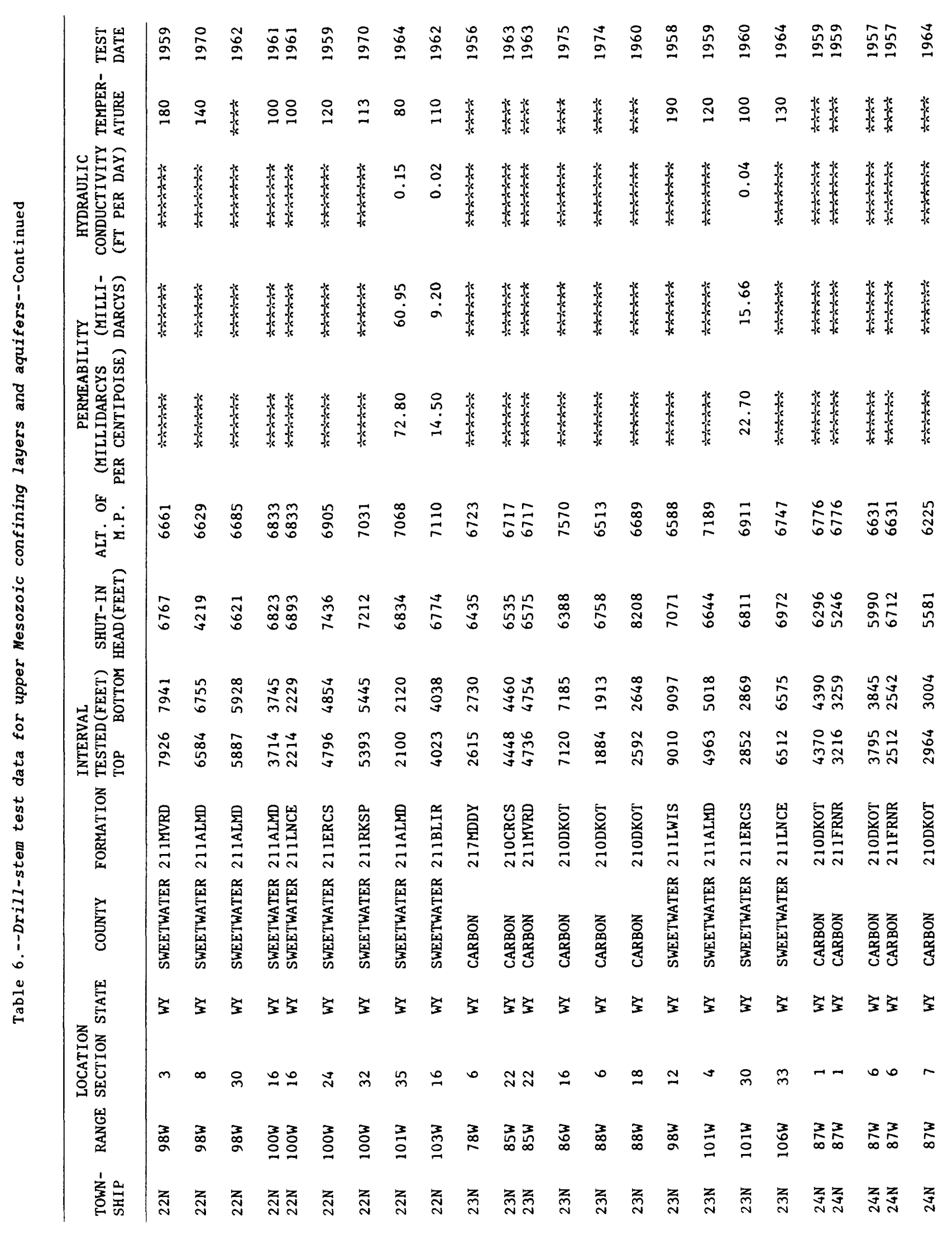




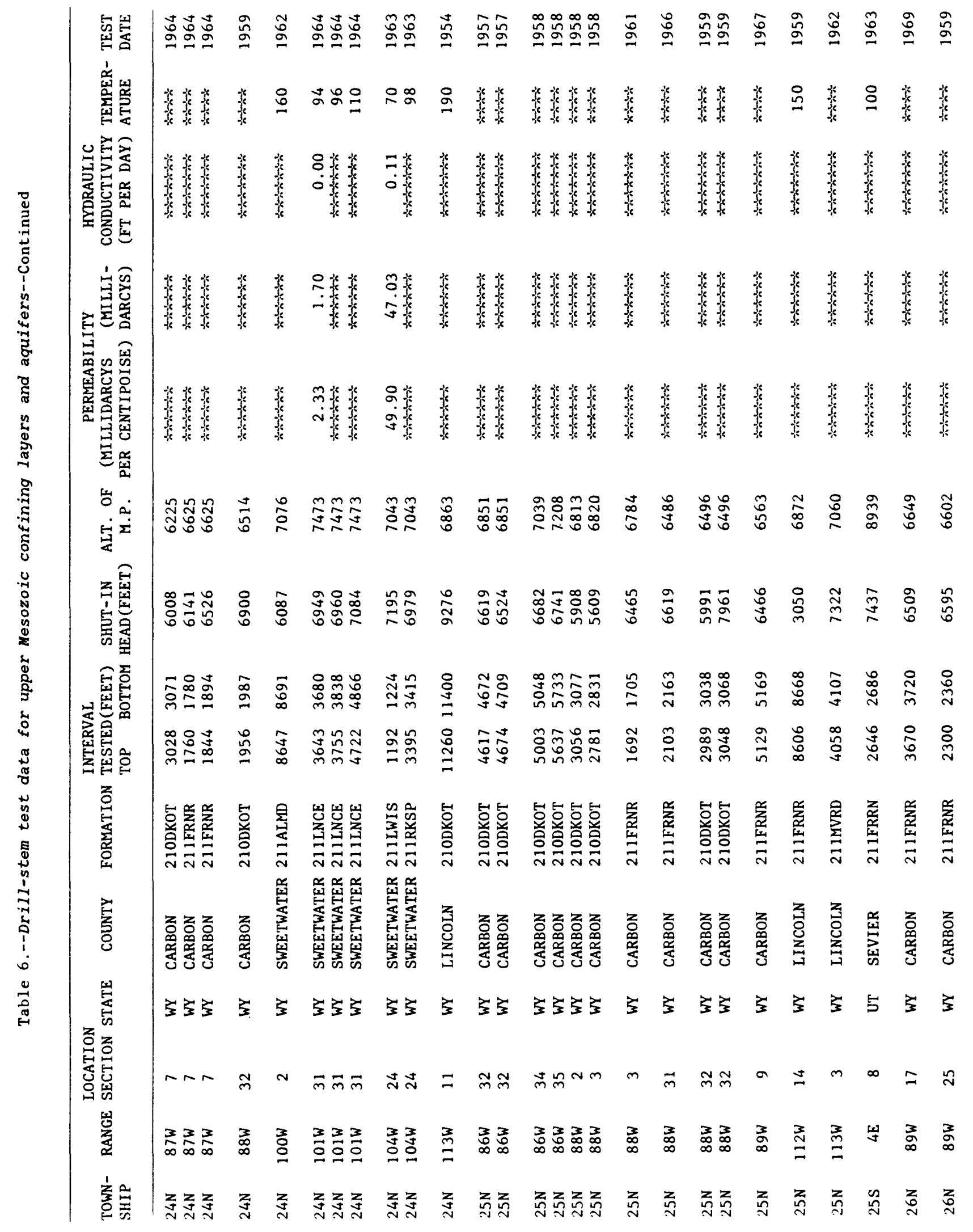




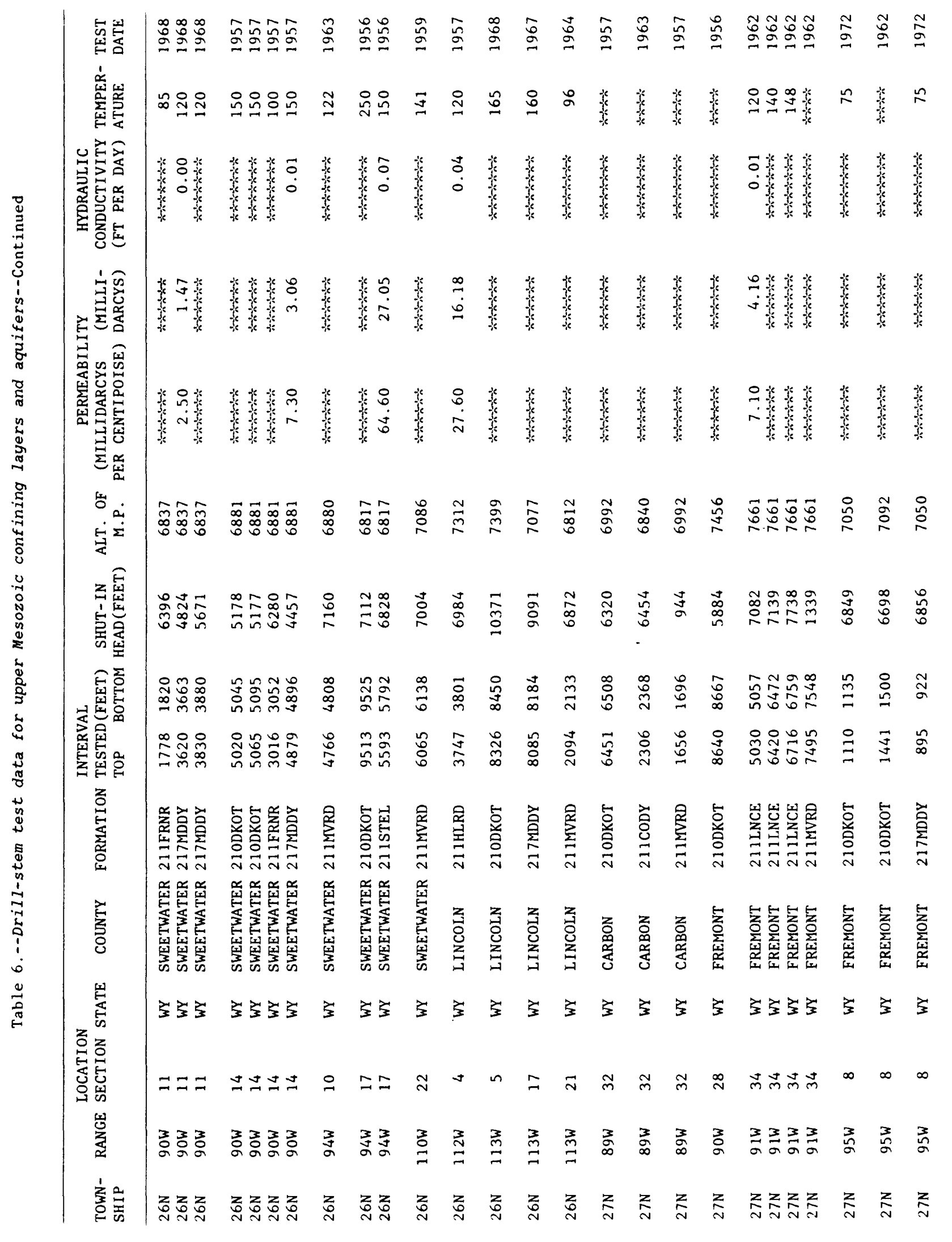




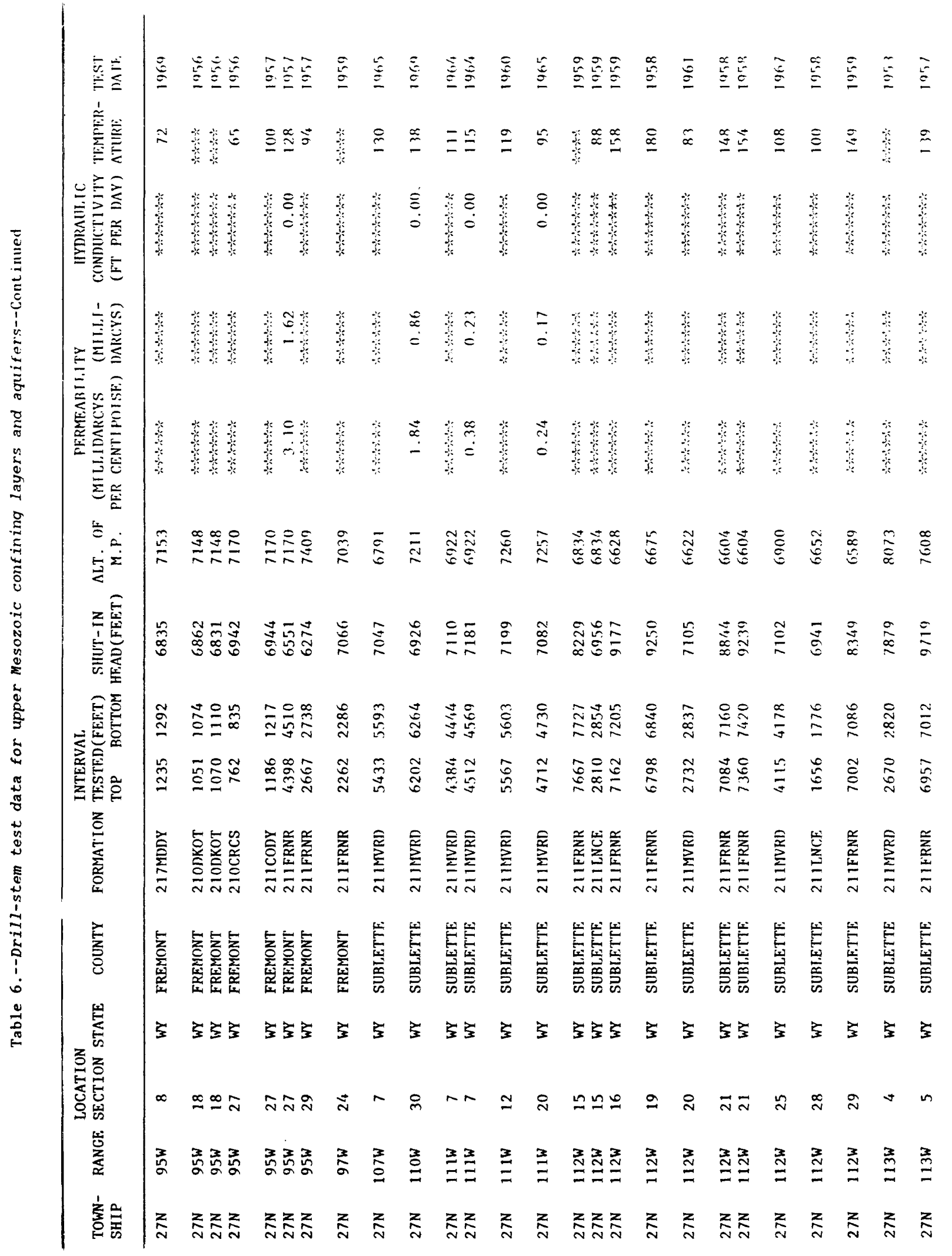




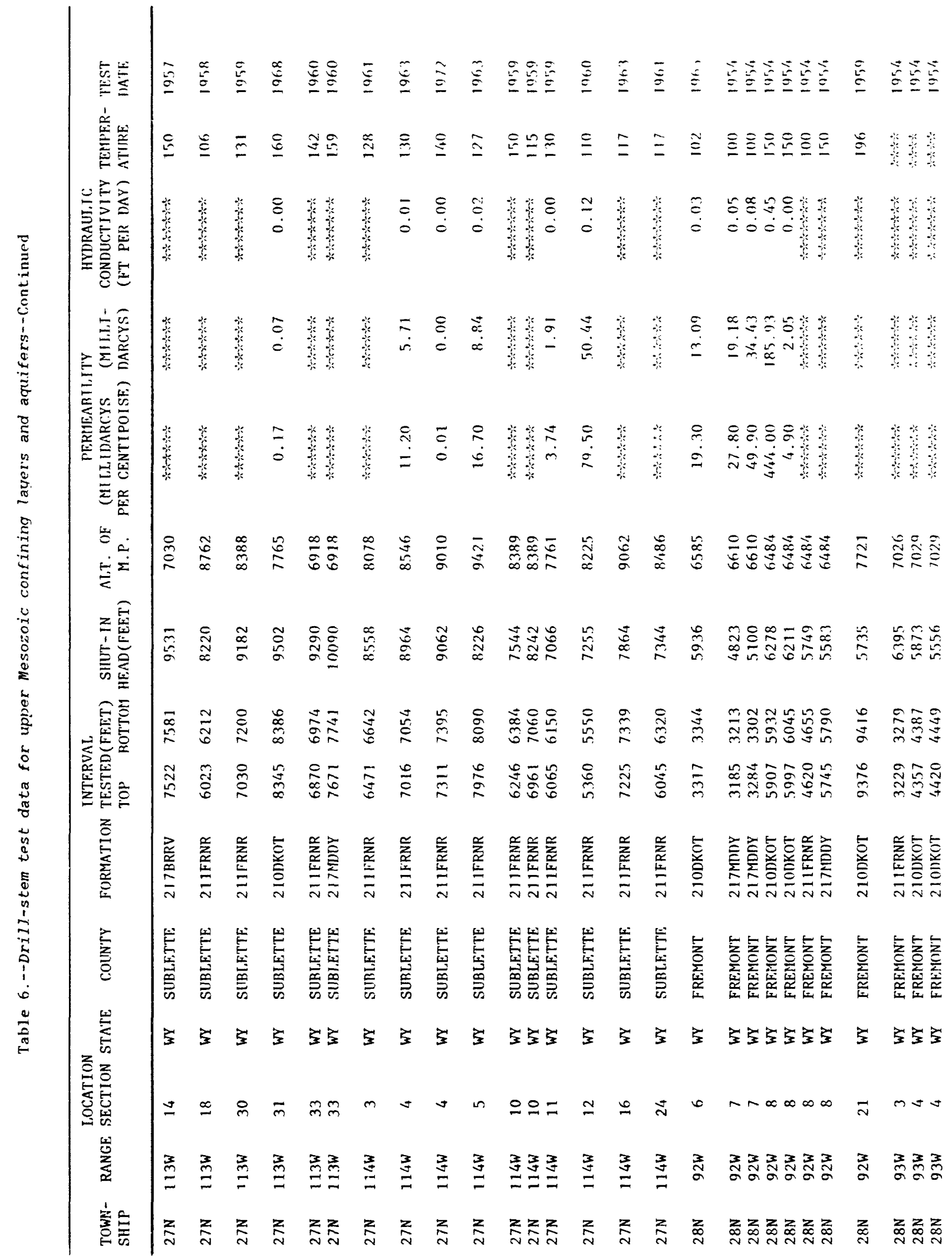




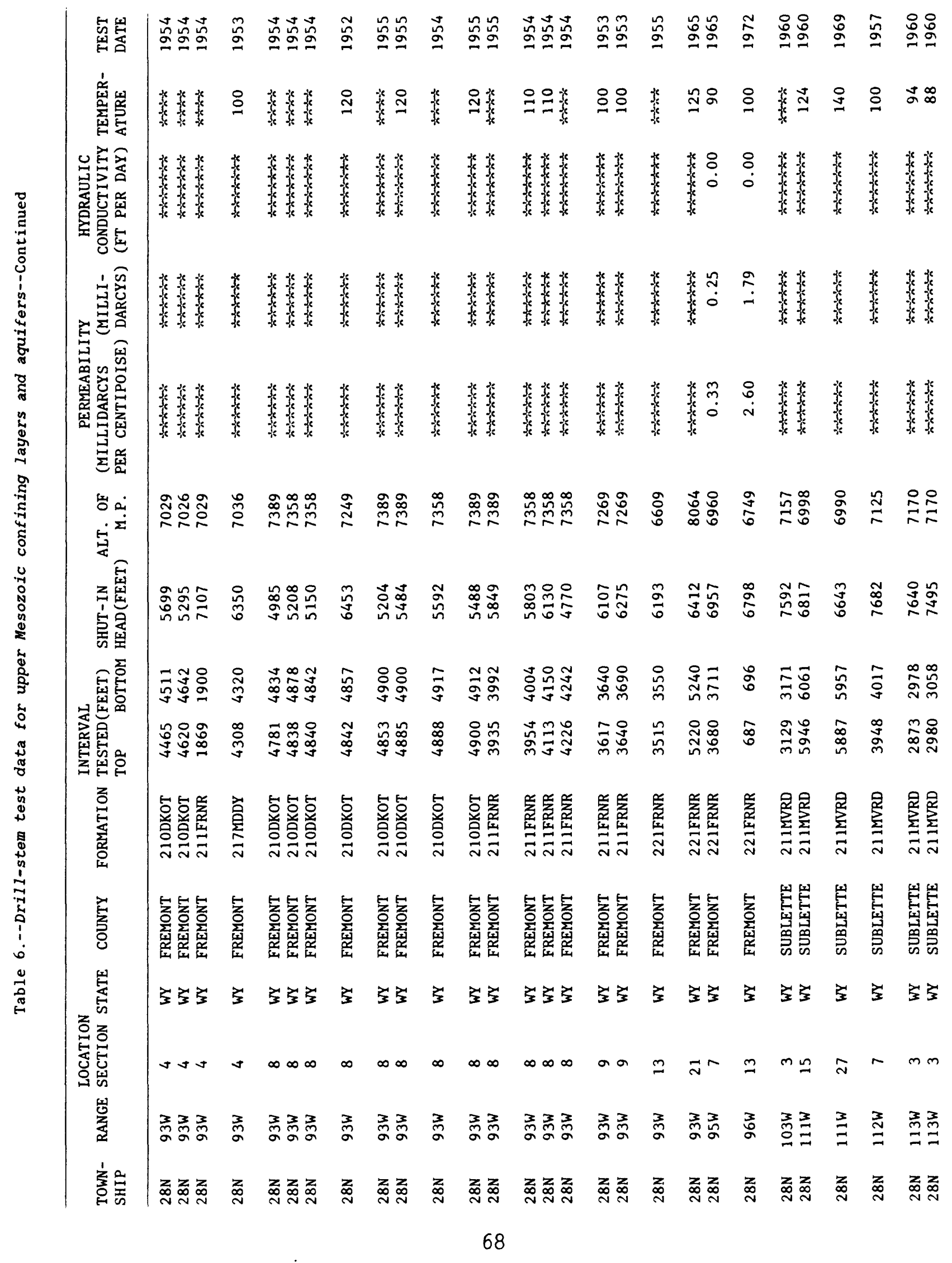




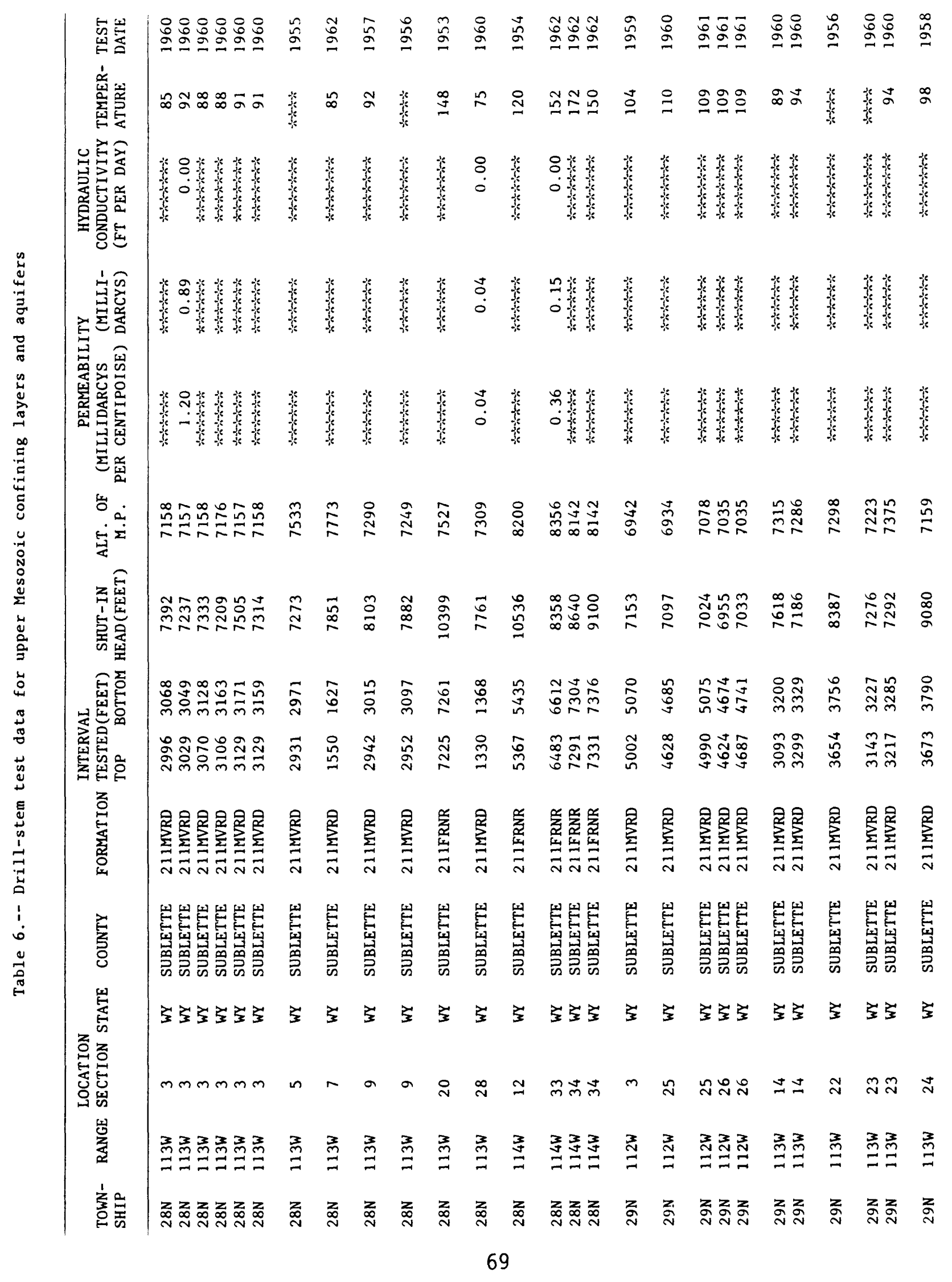




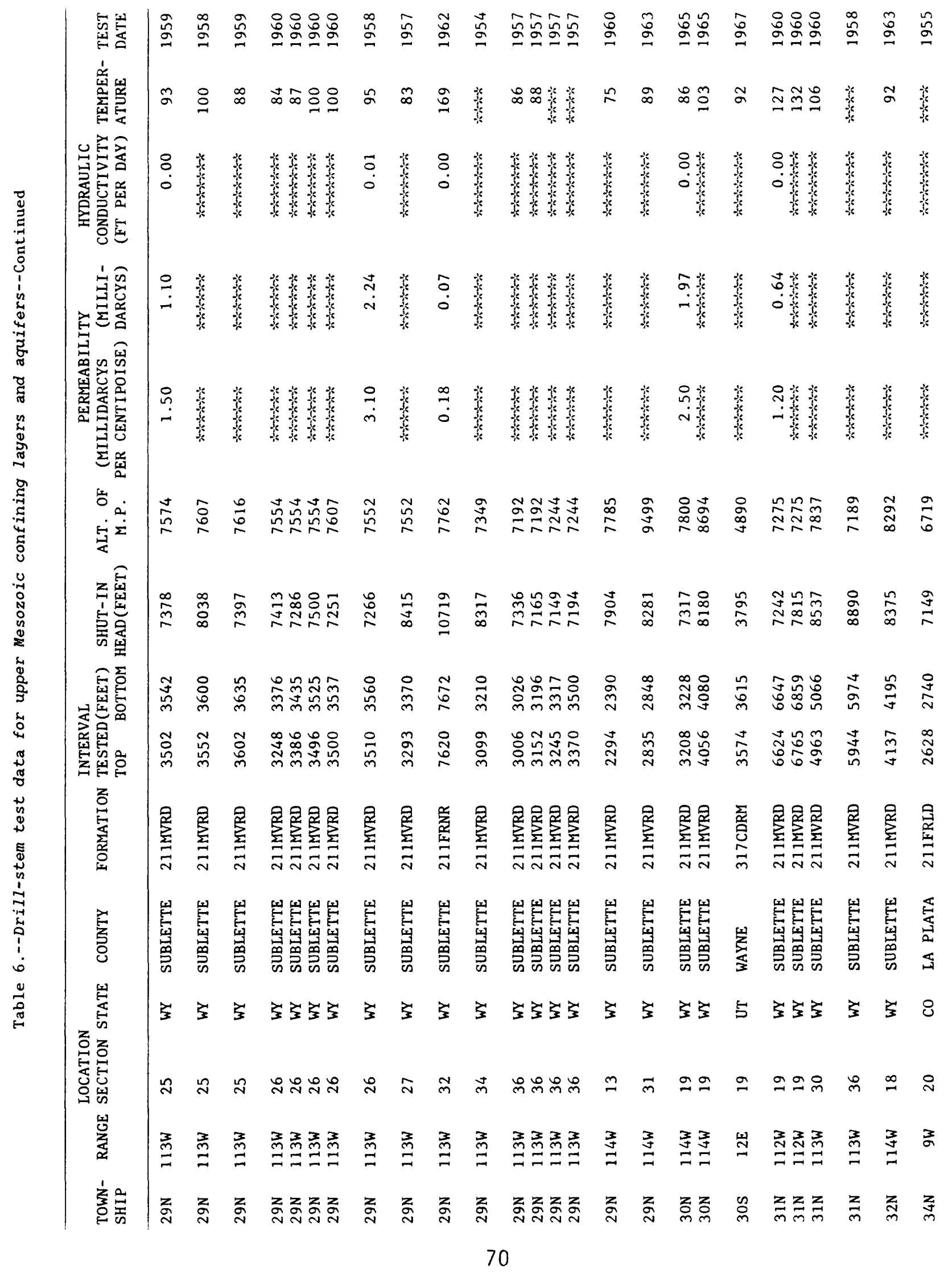




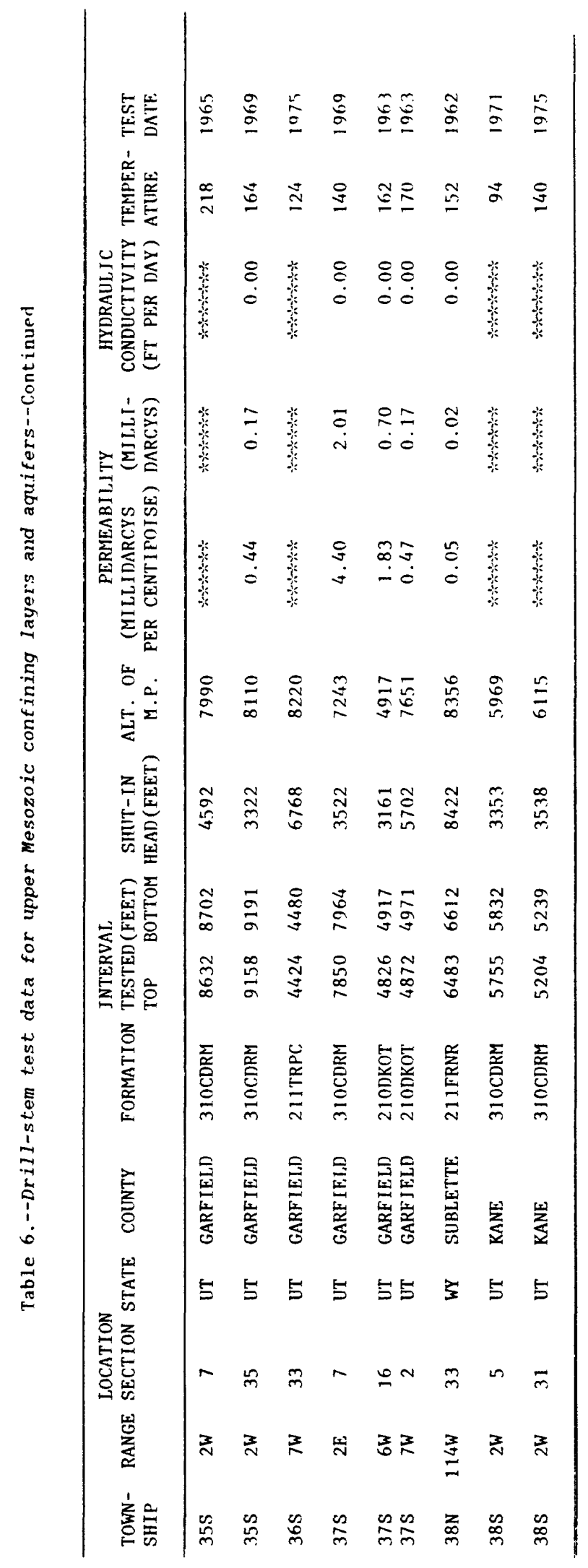




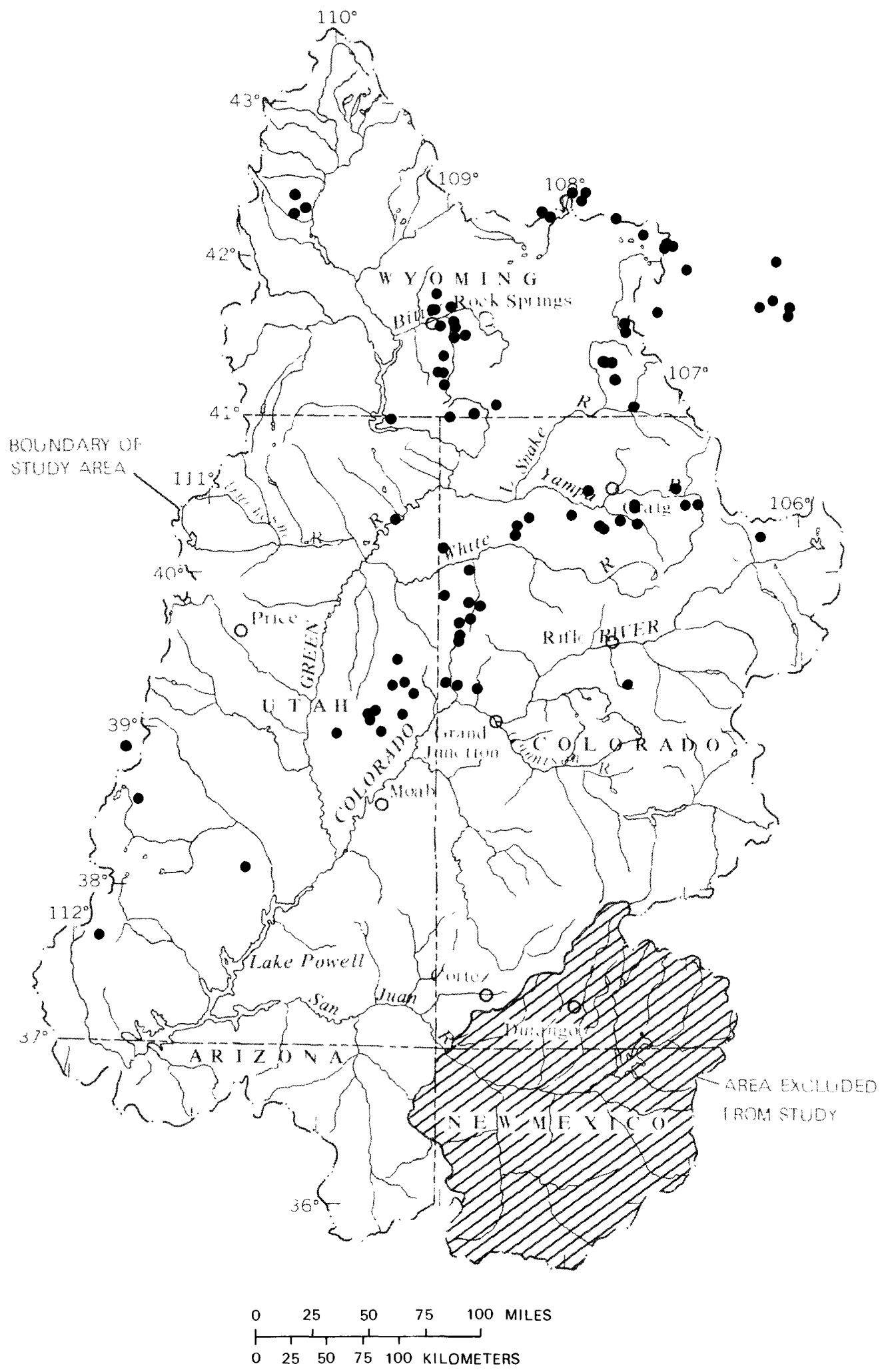

Figure 11.--Location of dri1l-stem test data for middle Mesozoic aquifers. 


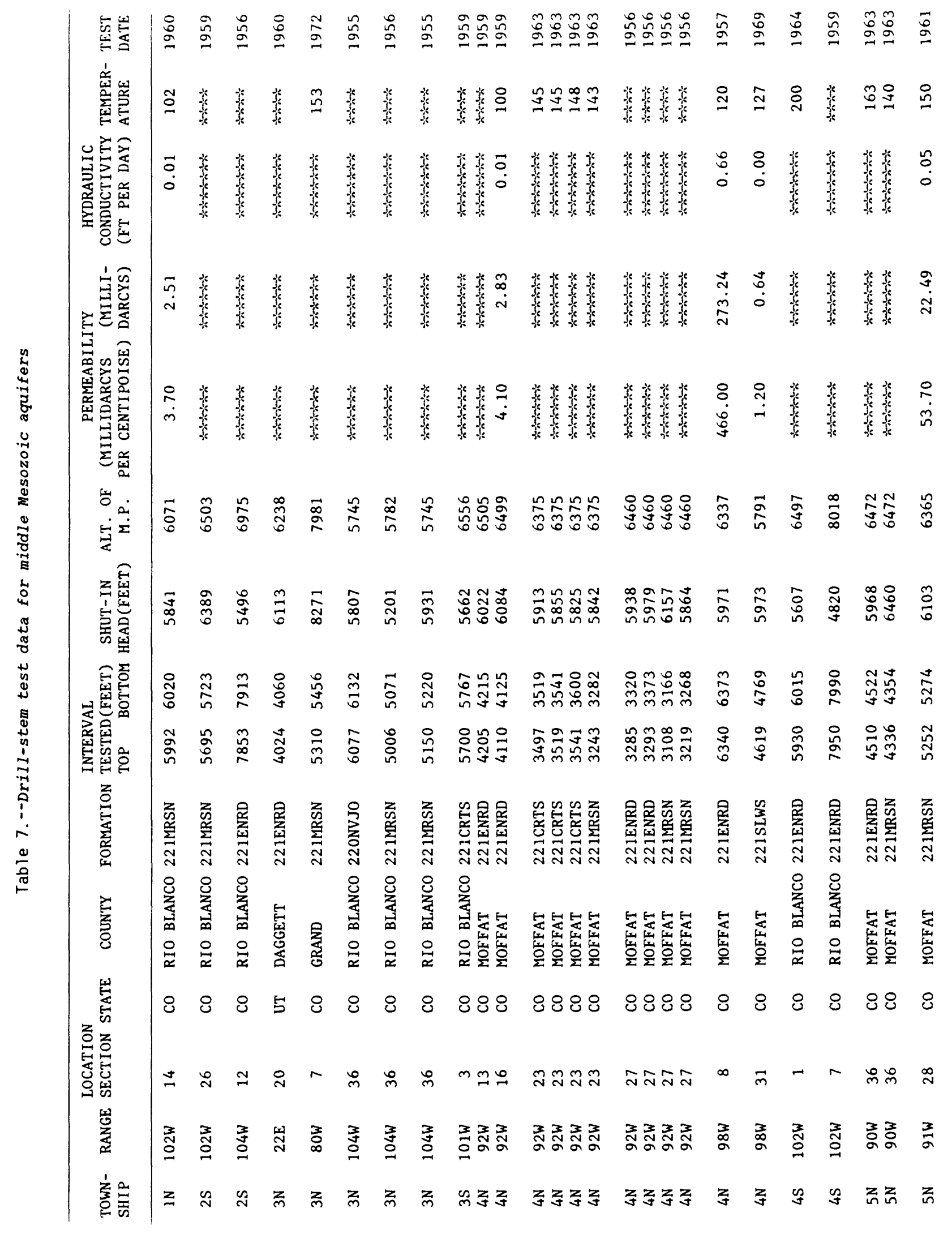




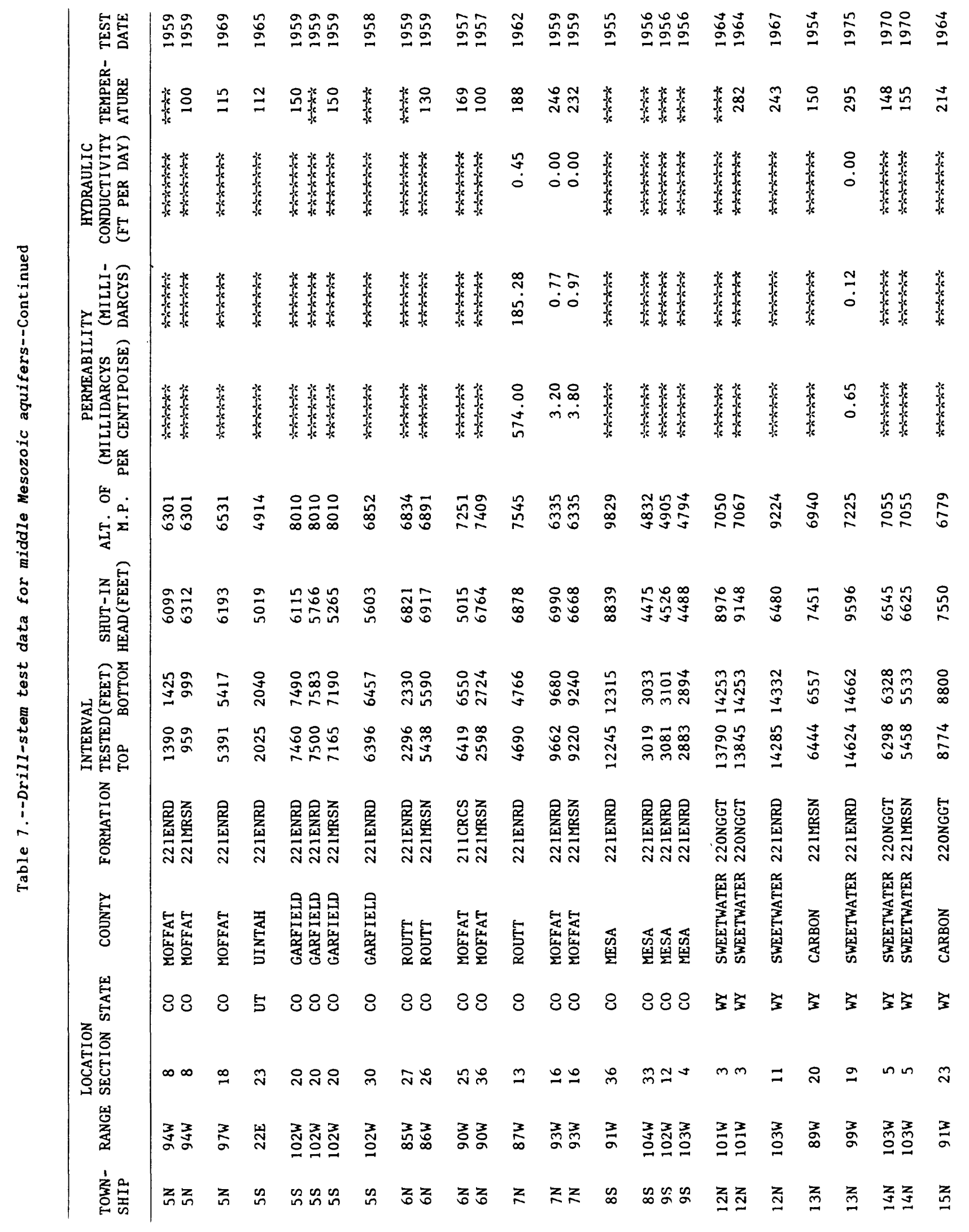




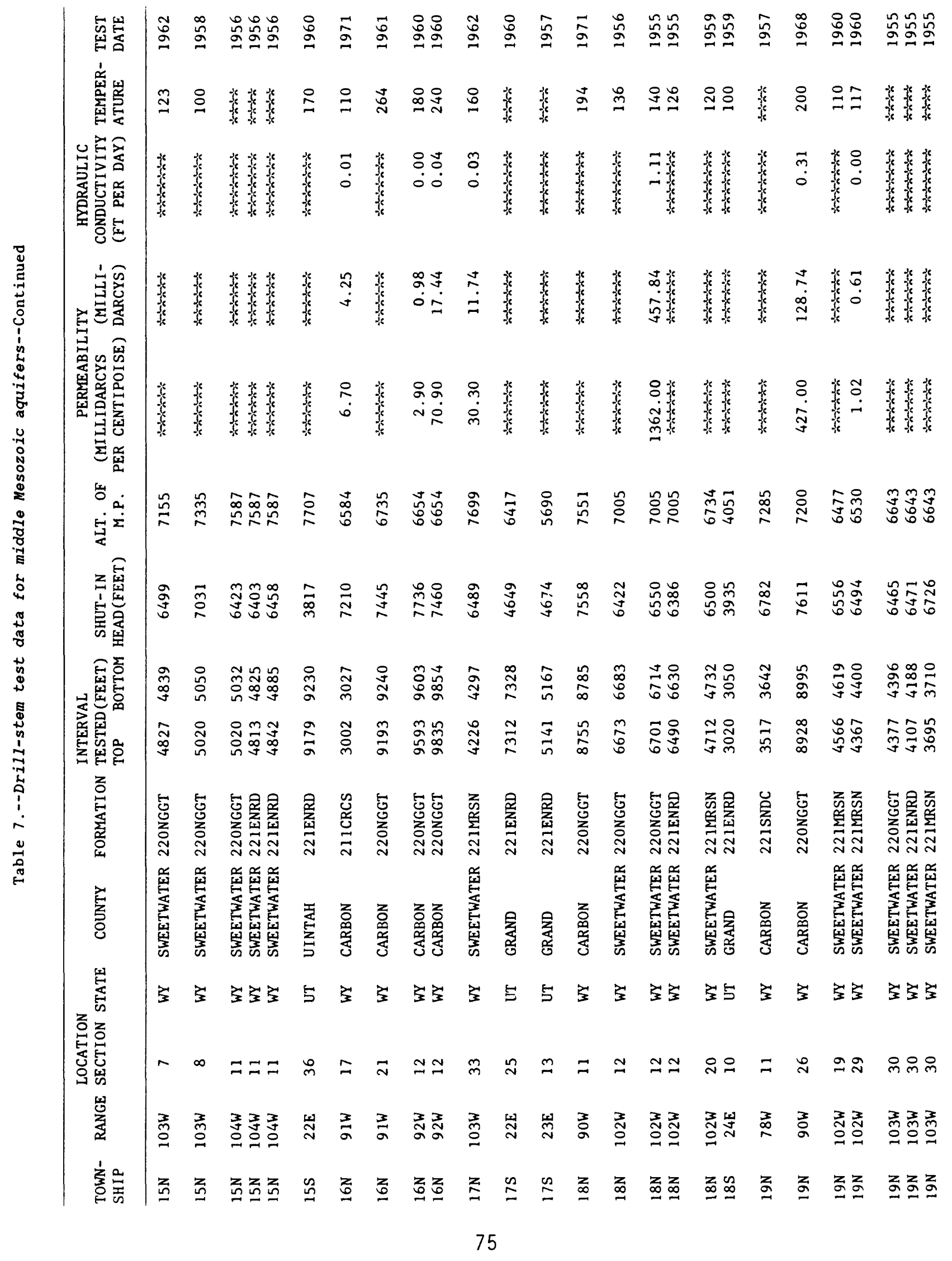




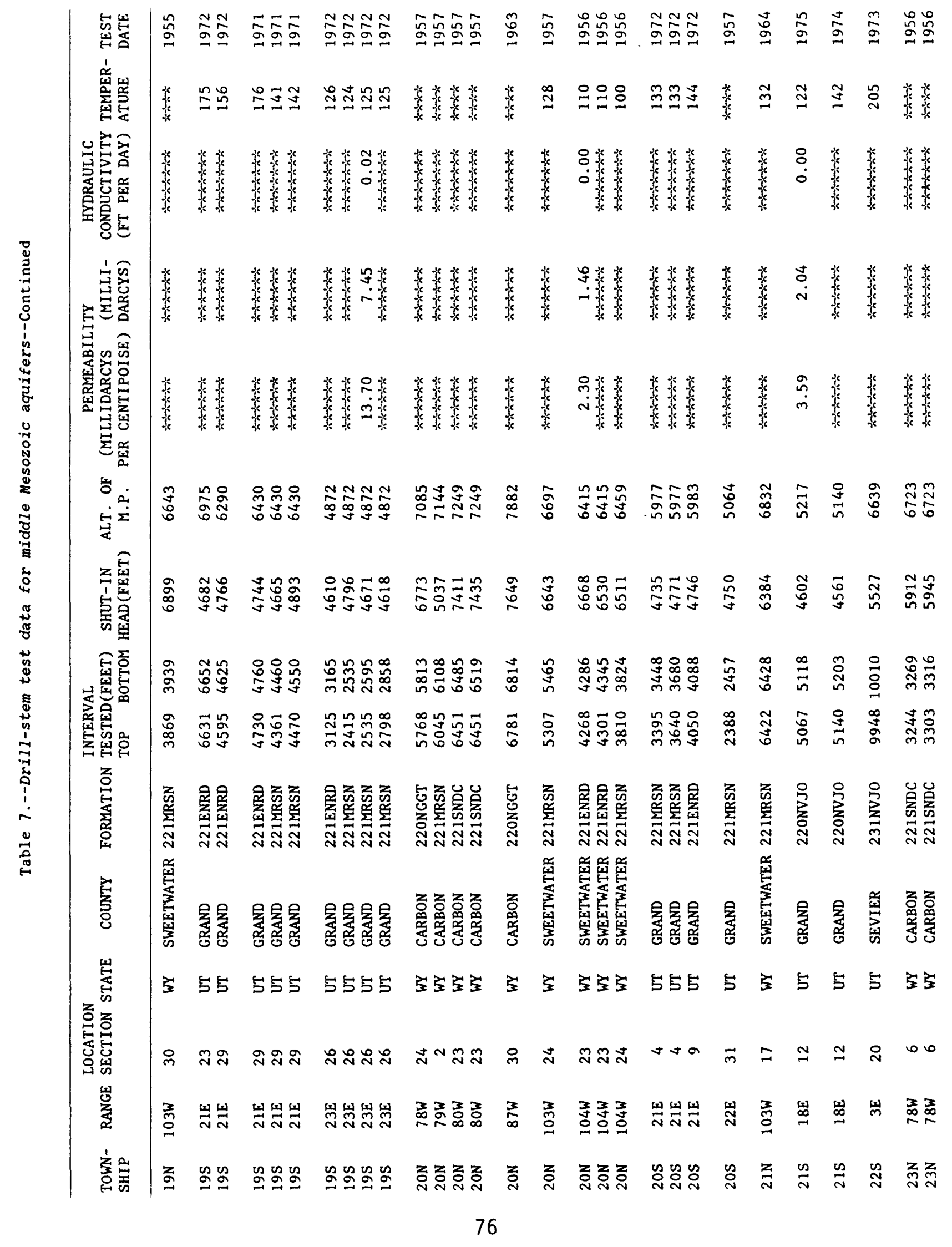




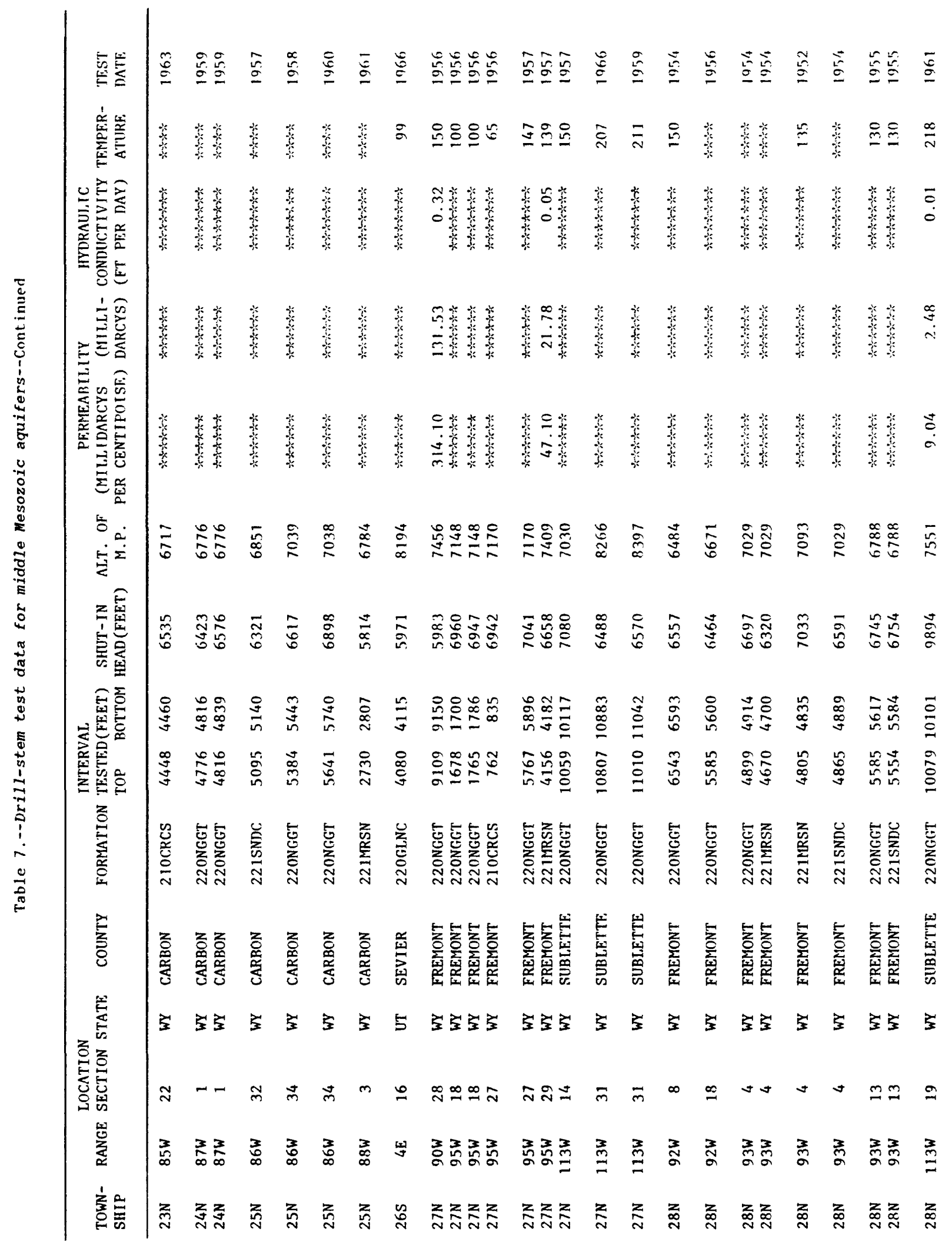




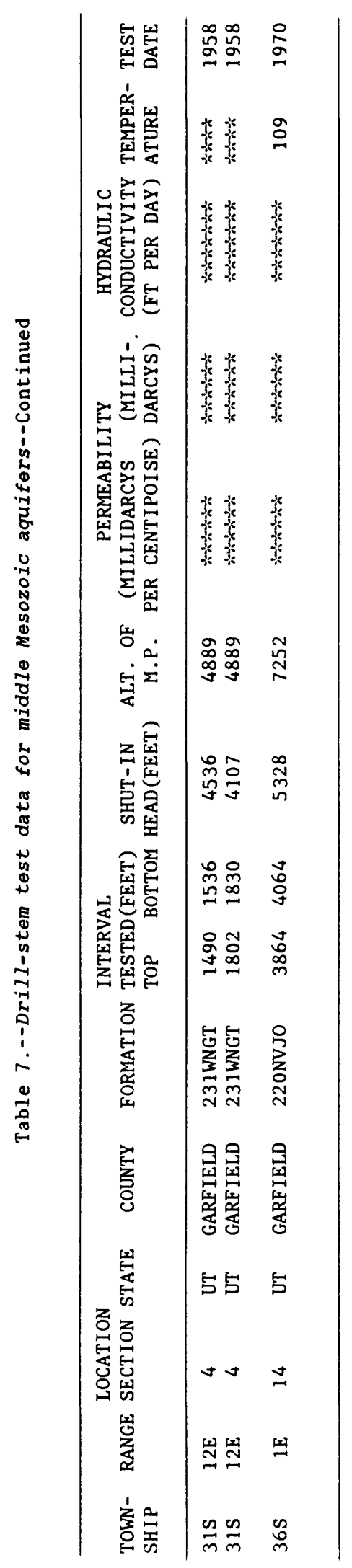




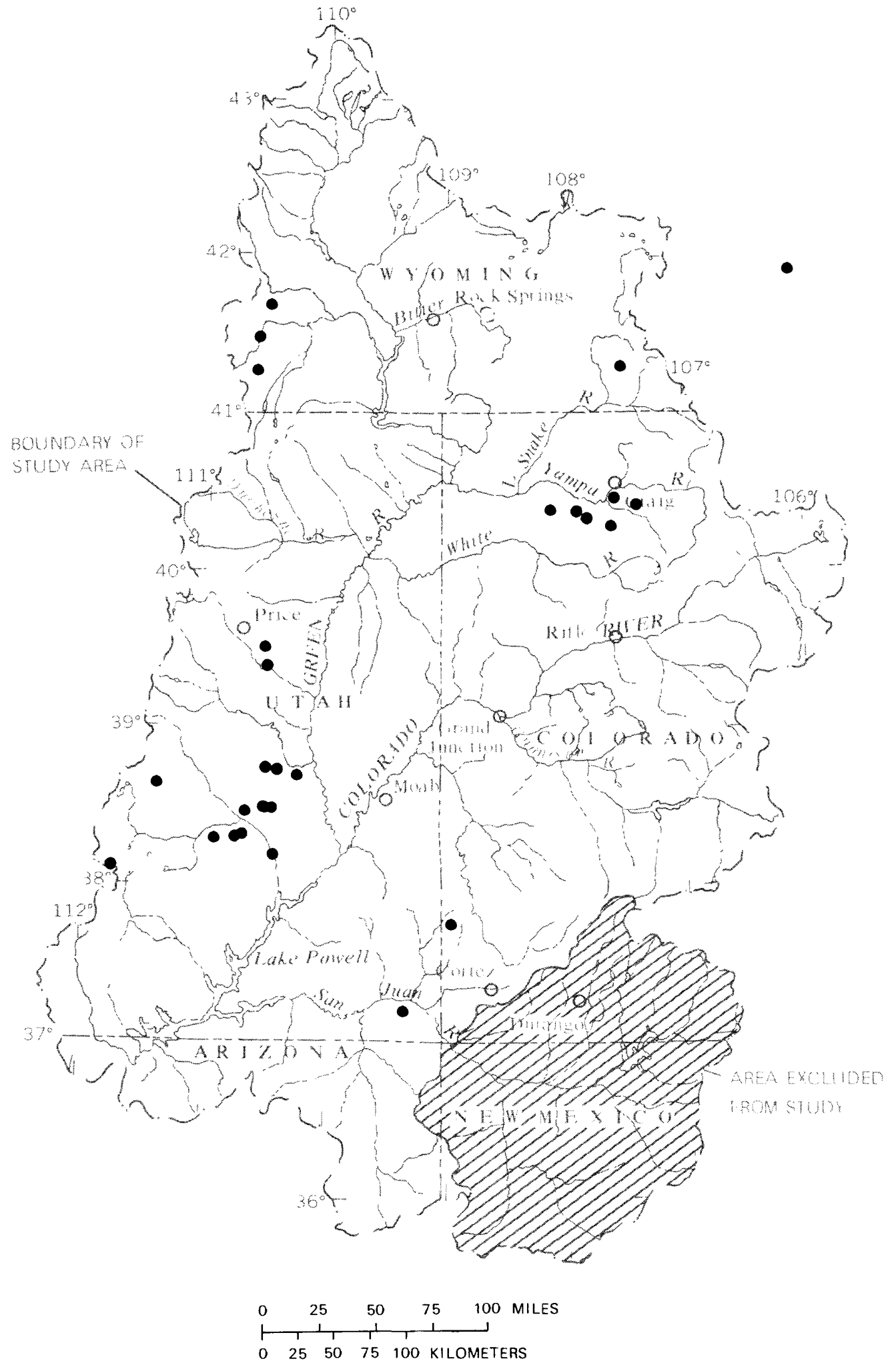

Figure 12.--Location of dril1-stem test data for lower Mesozoic confining layers. 


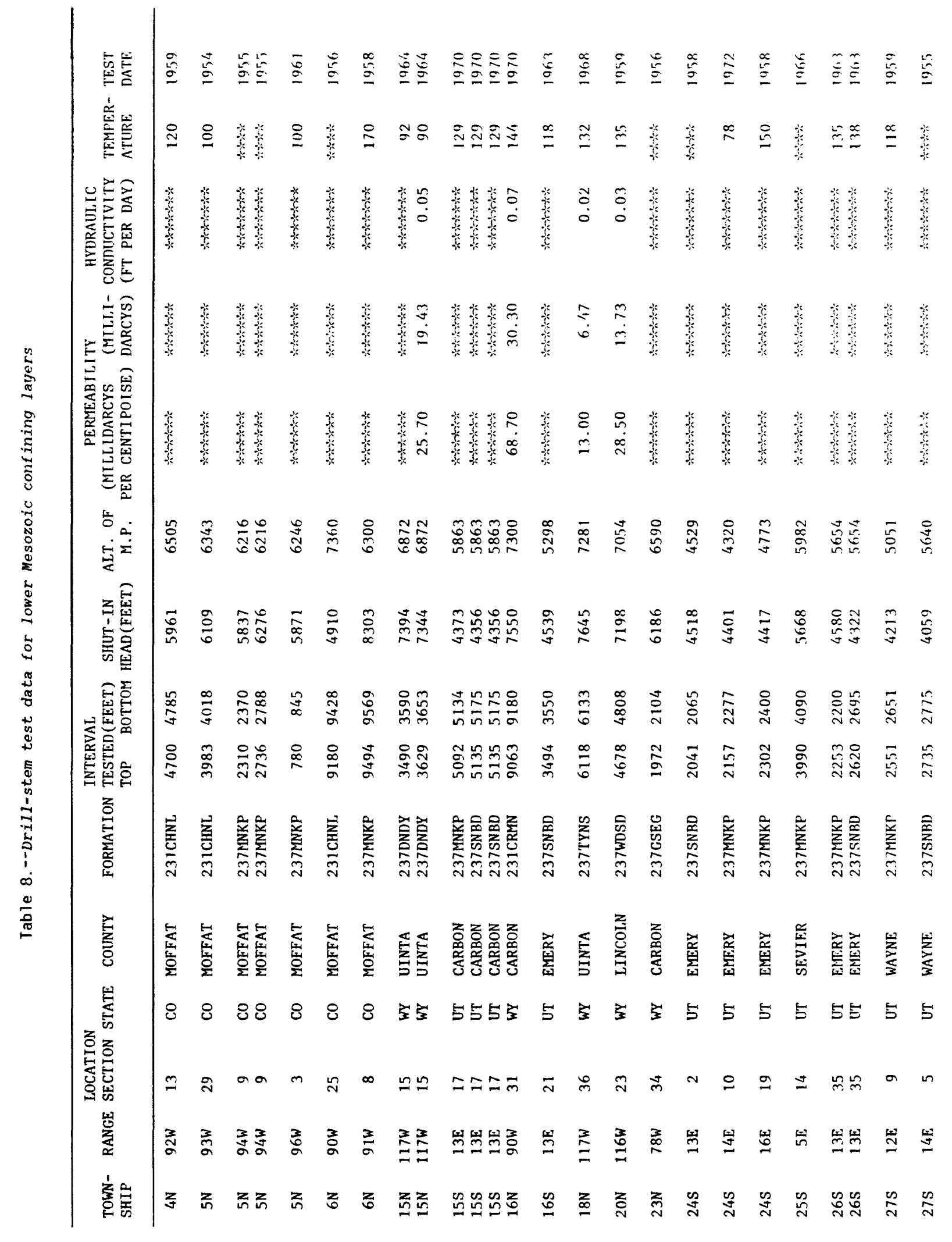




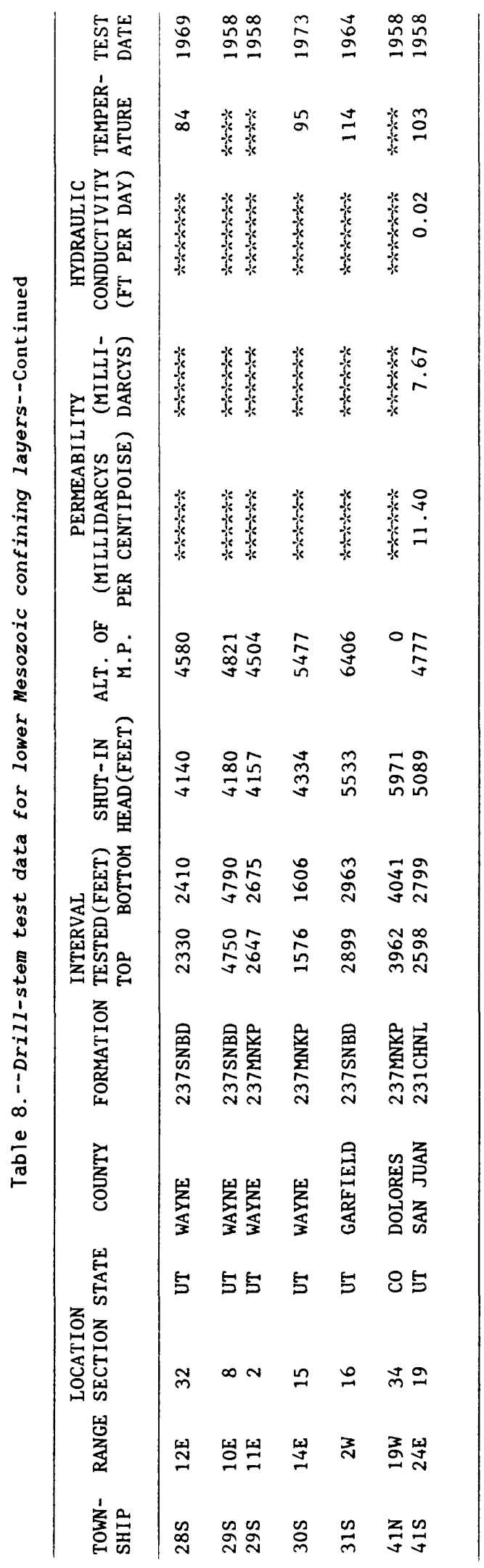




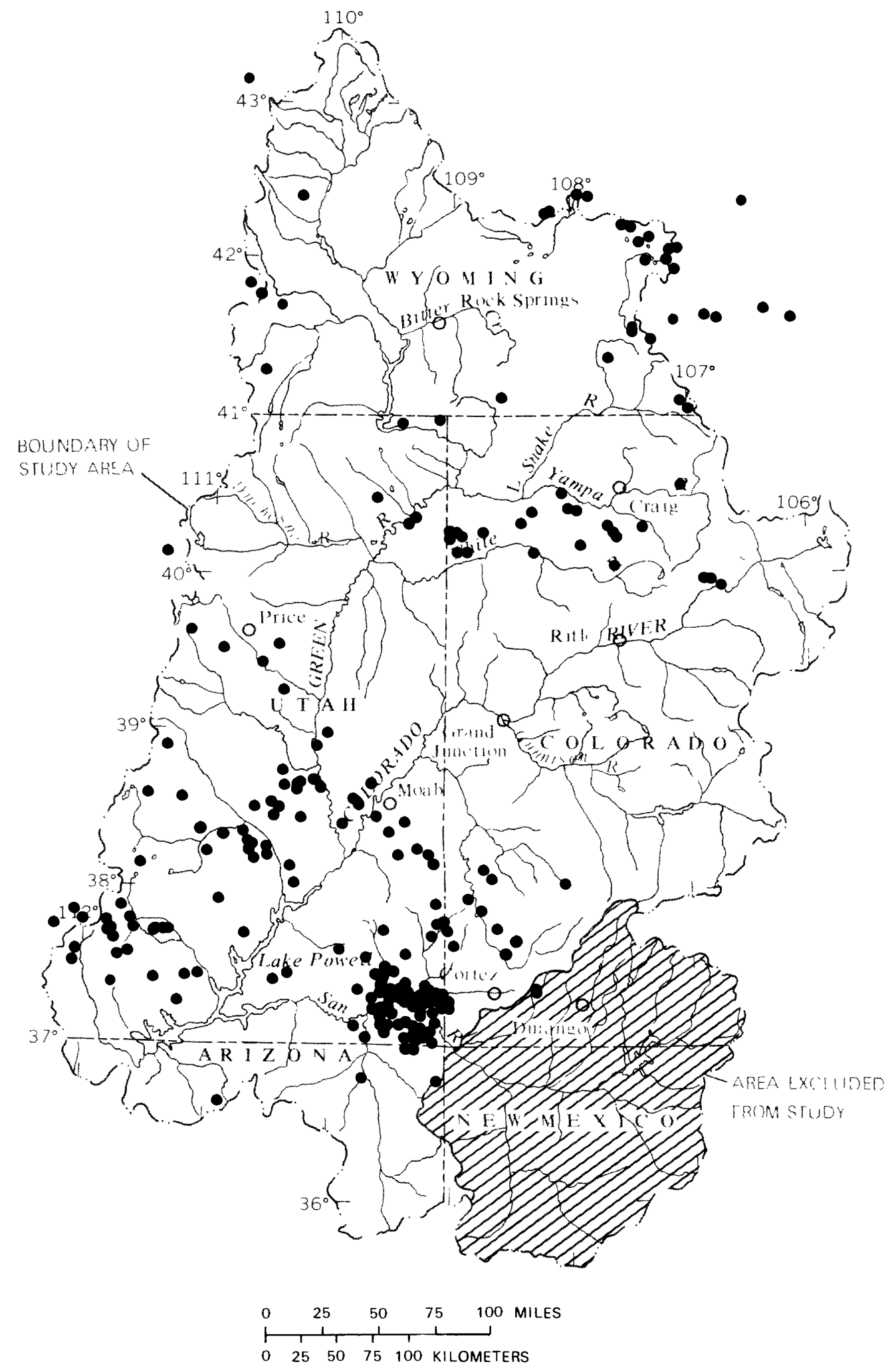

Figure 13.--Location of dri11-stem test data for upper Paleozoic aquifers and confining layers. 


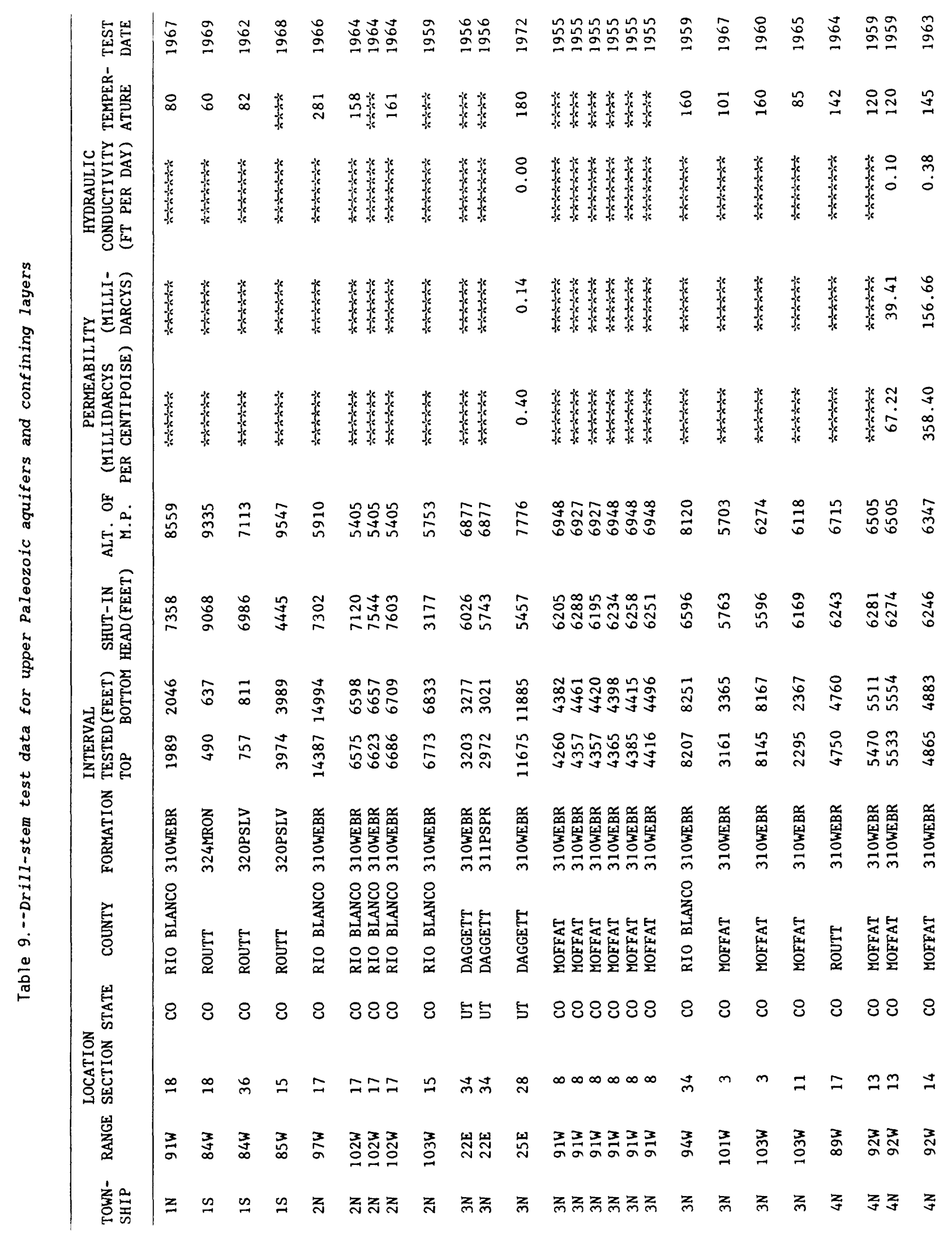




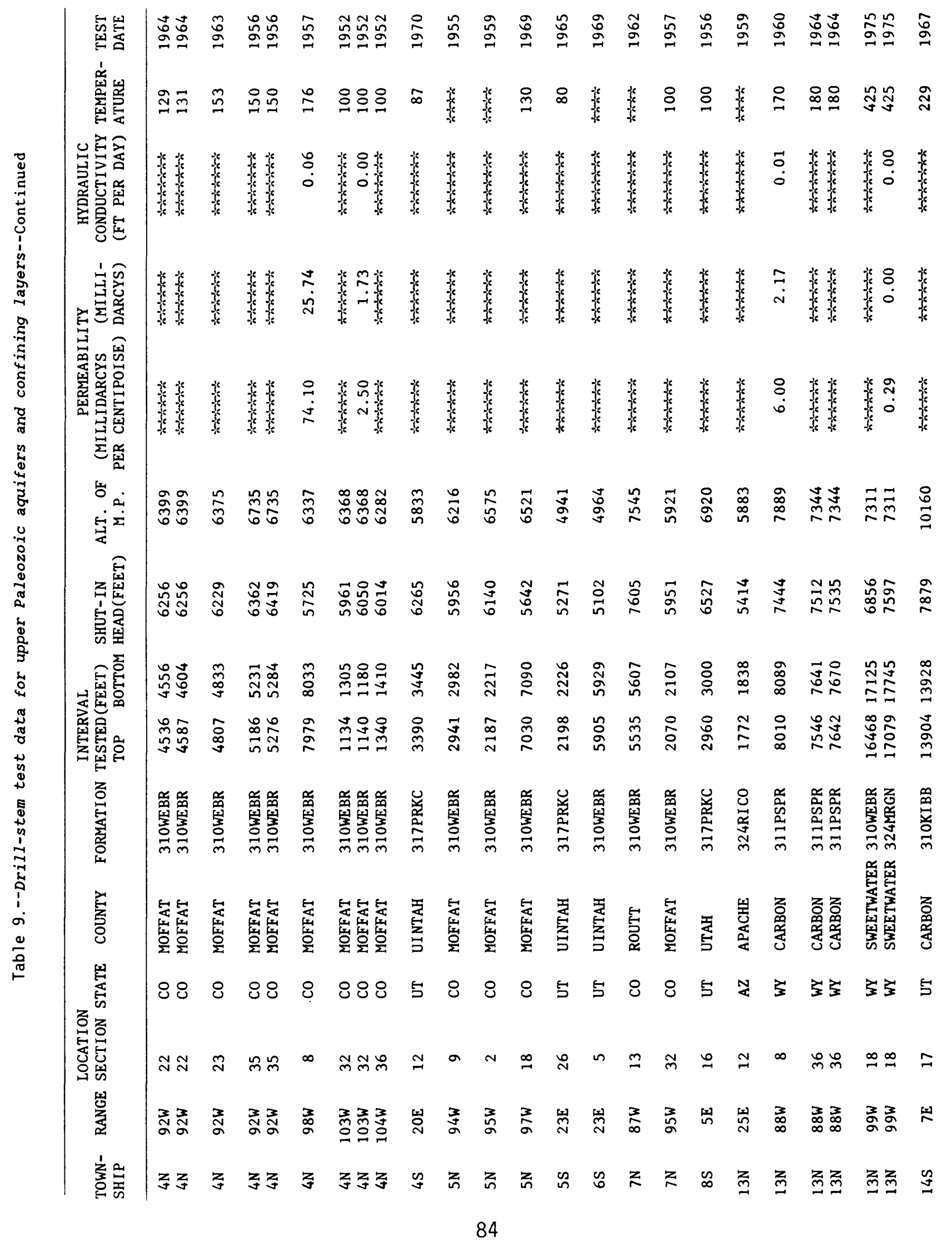




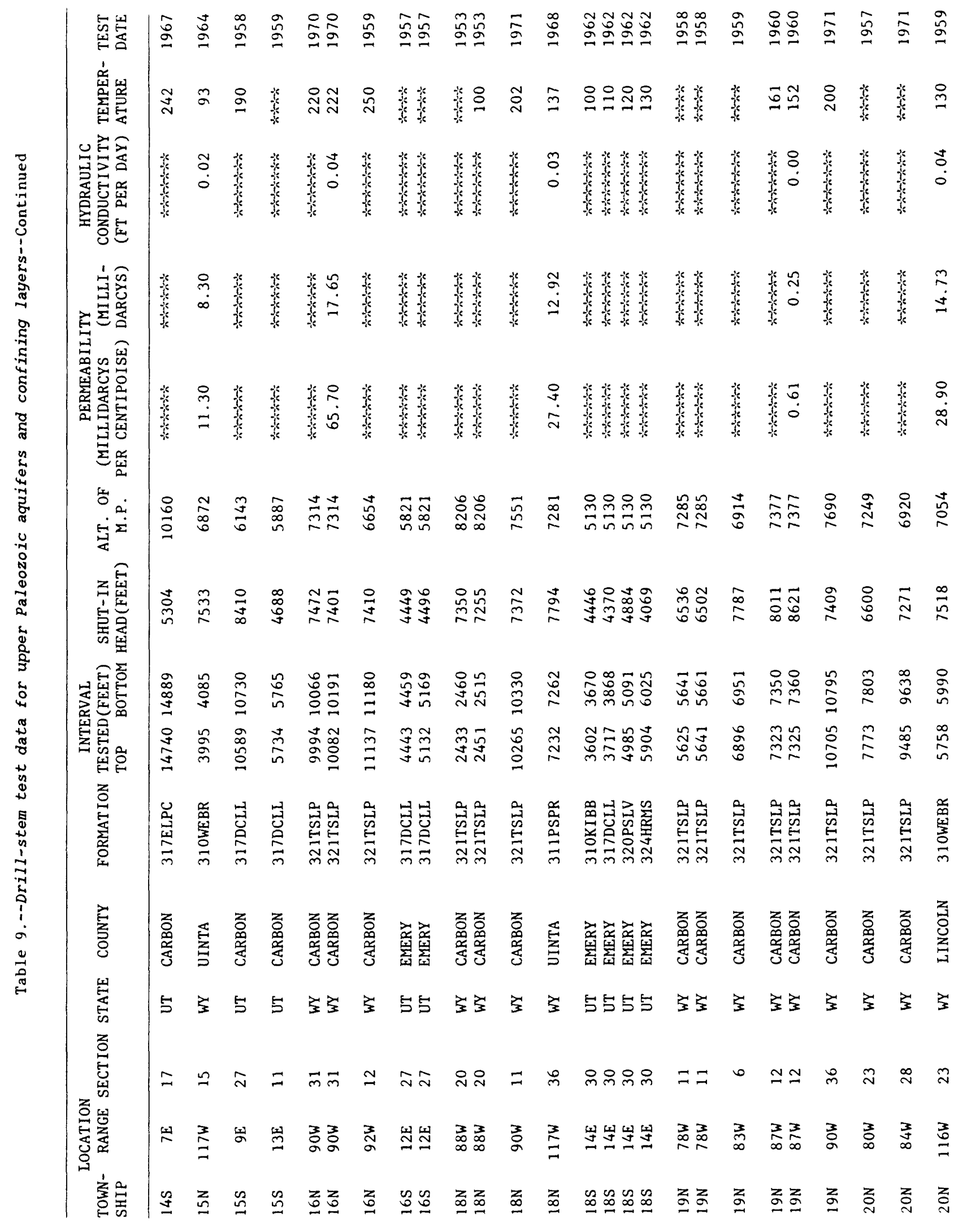




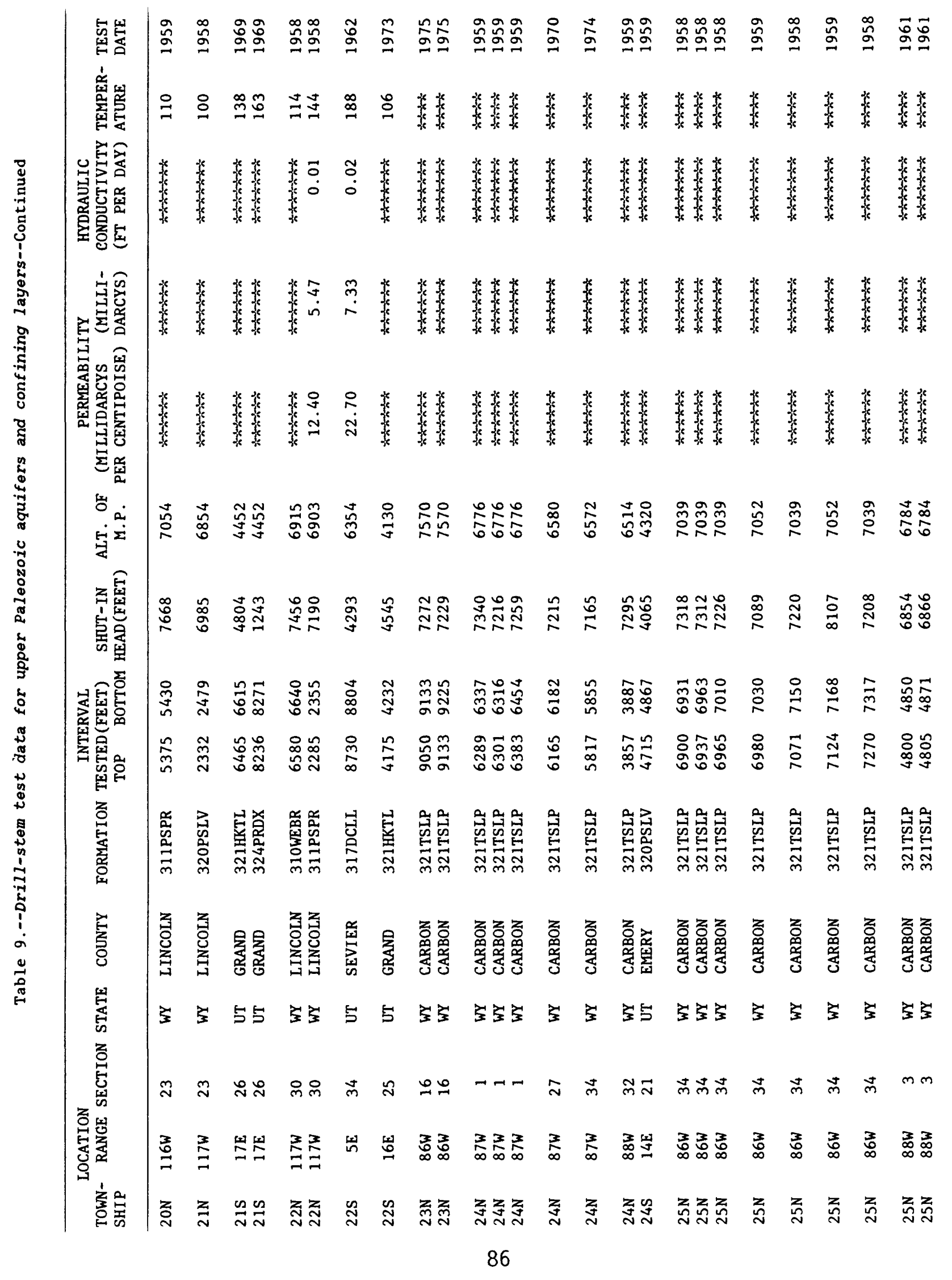




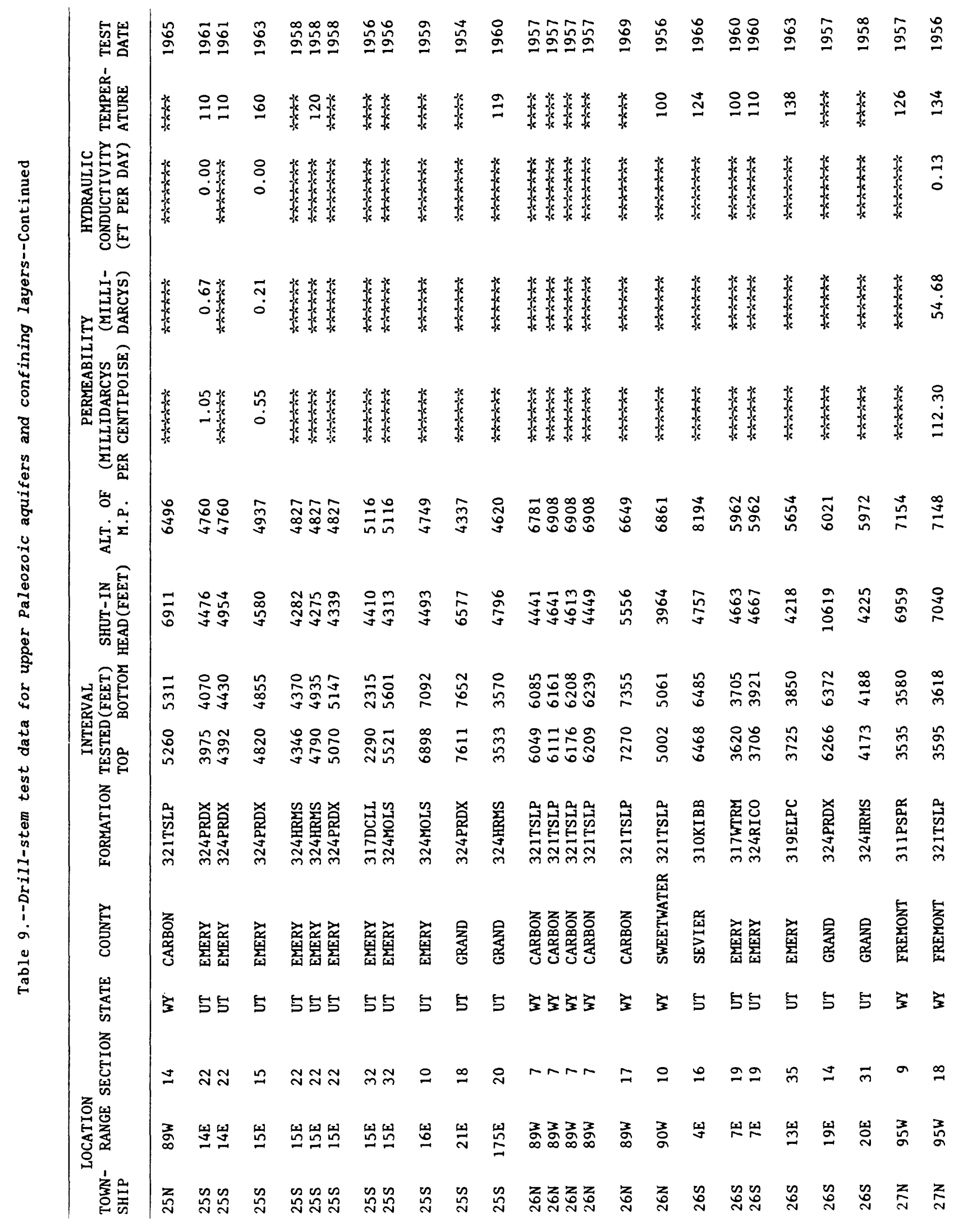




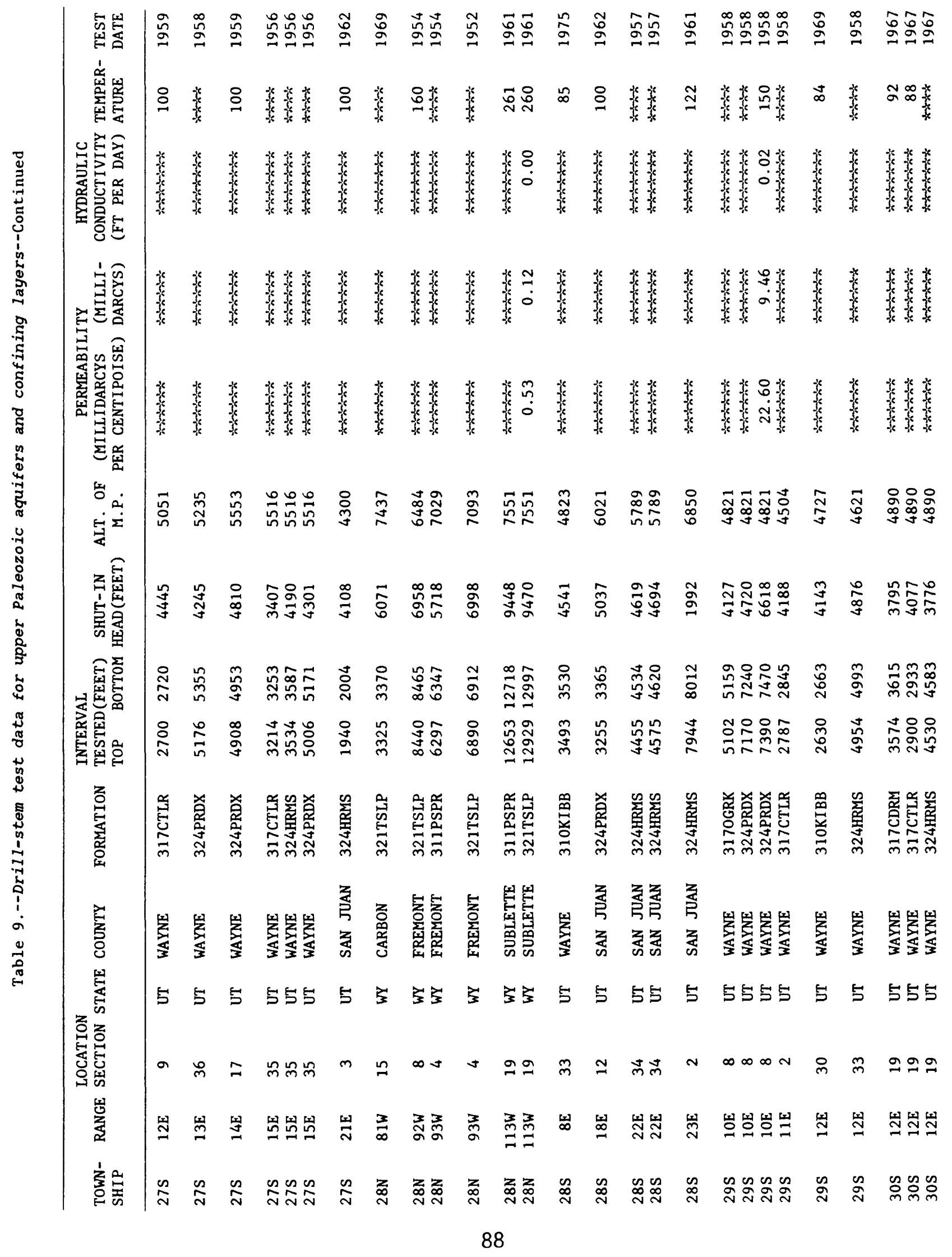




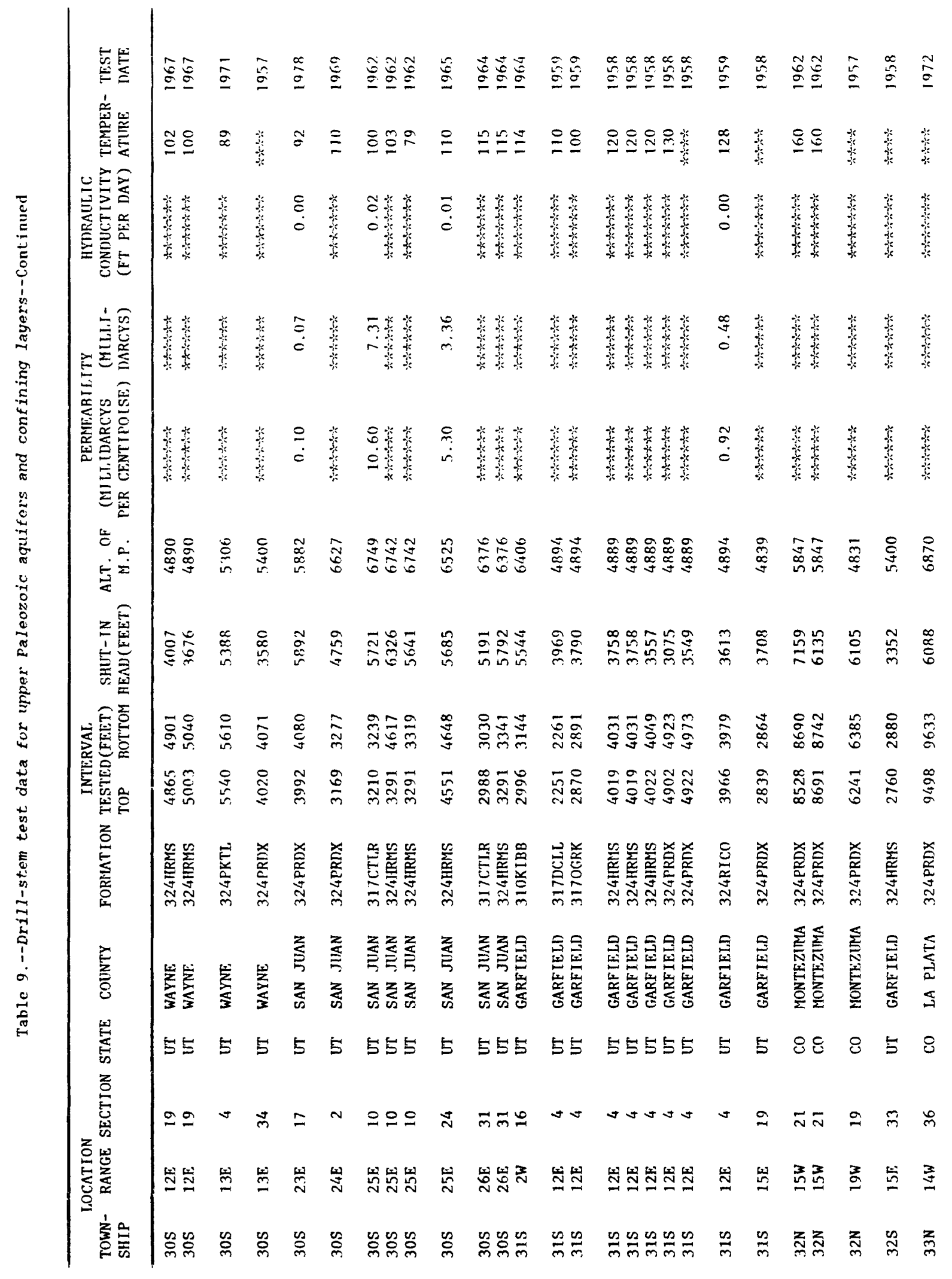




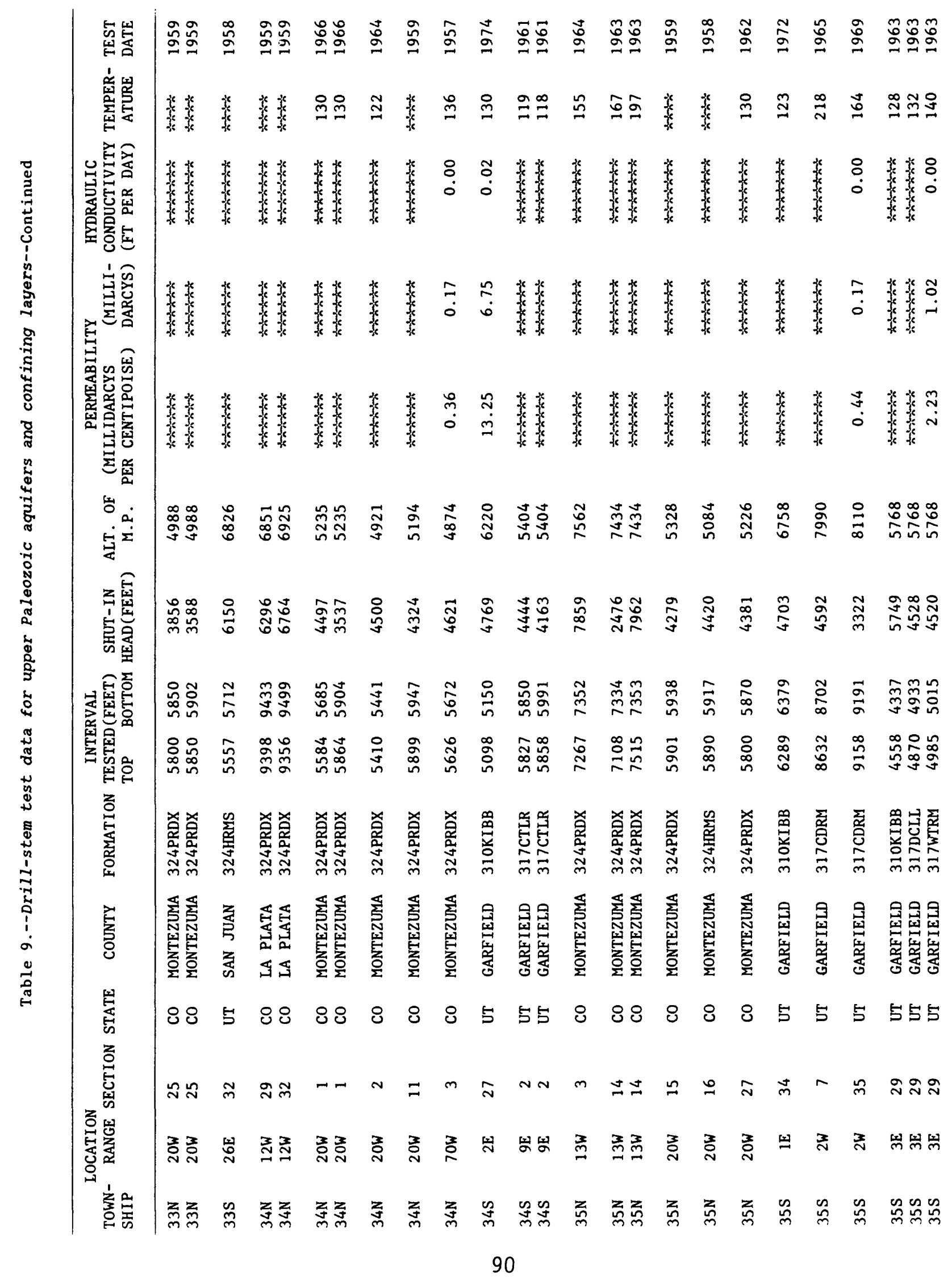




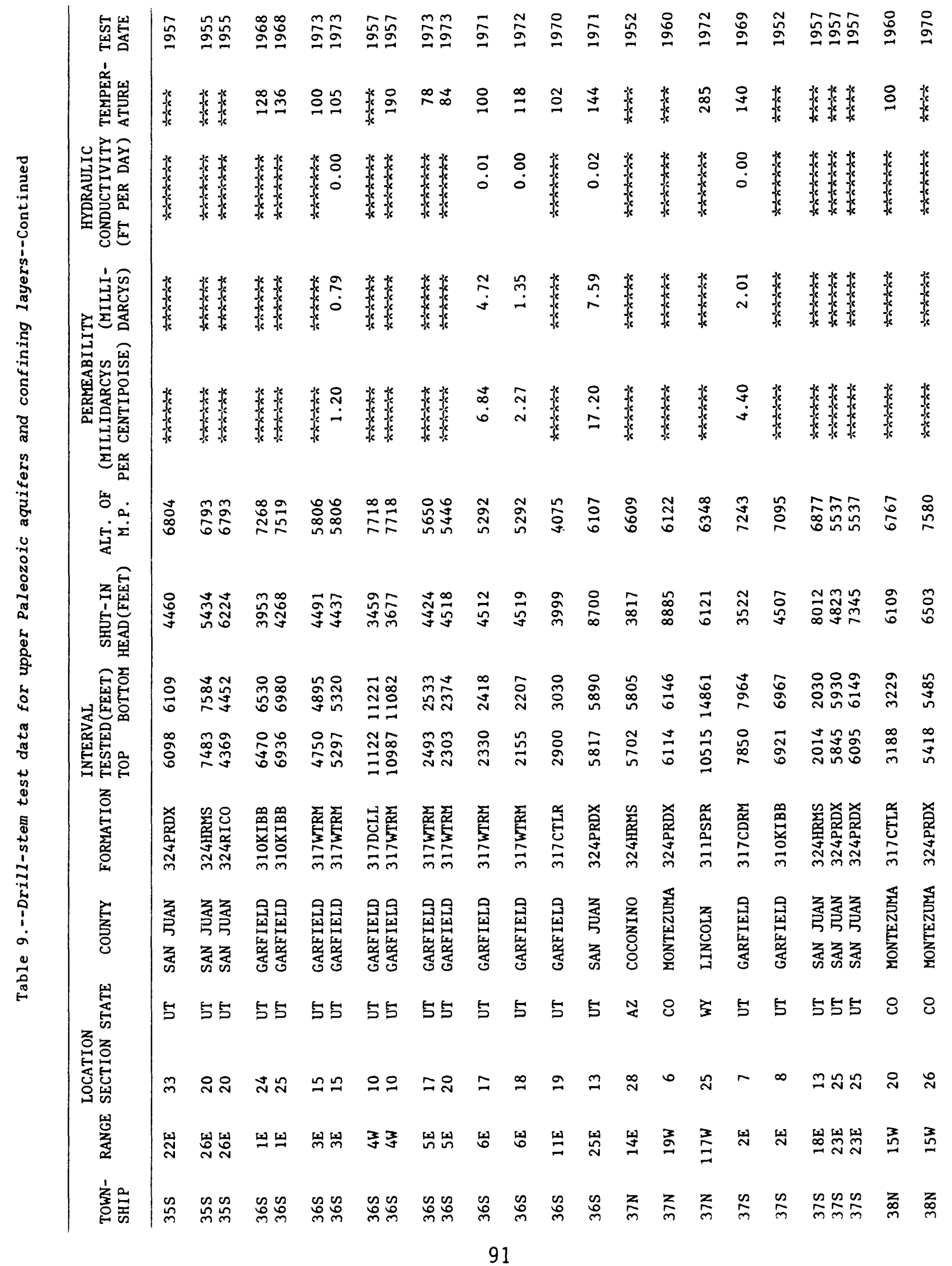




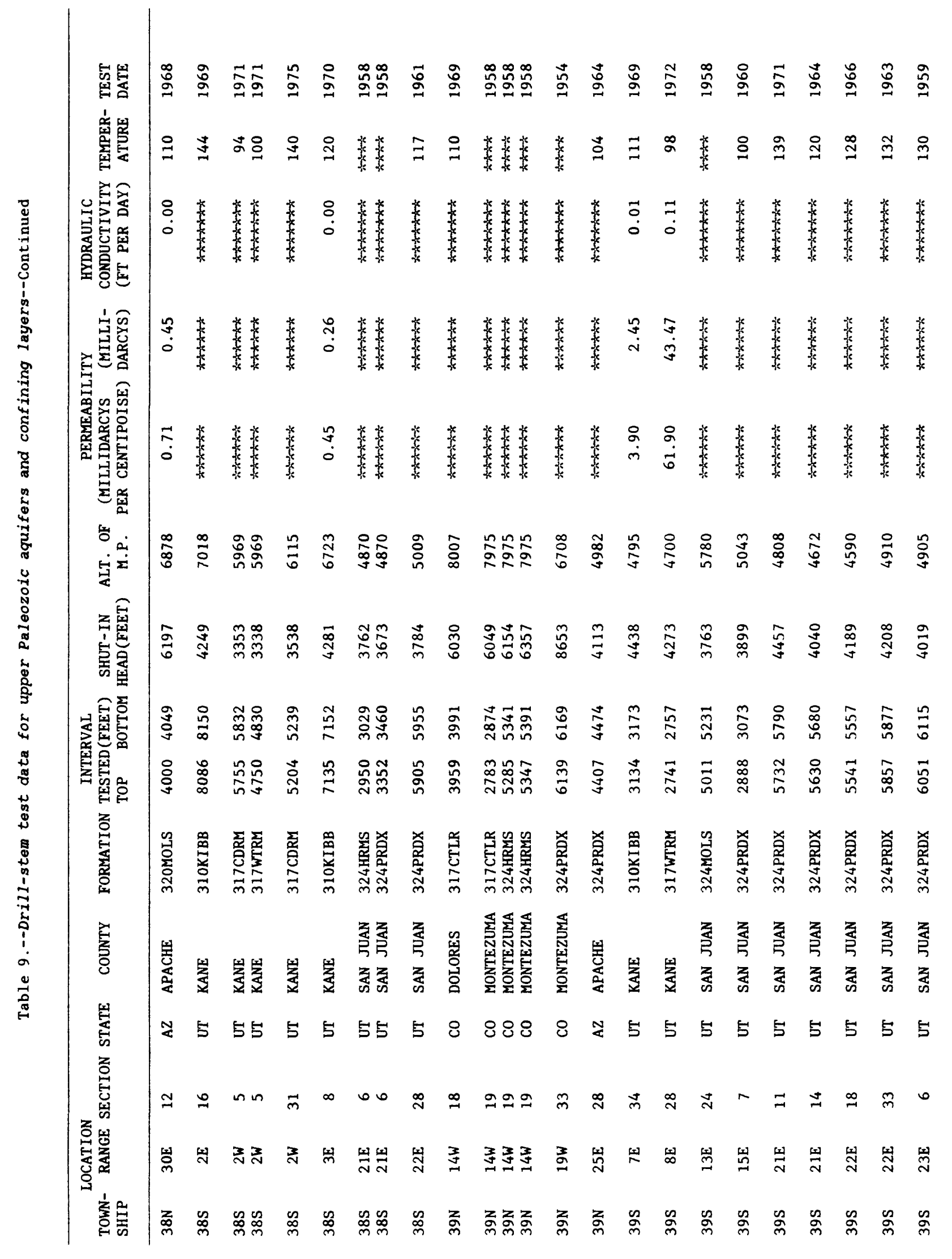




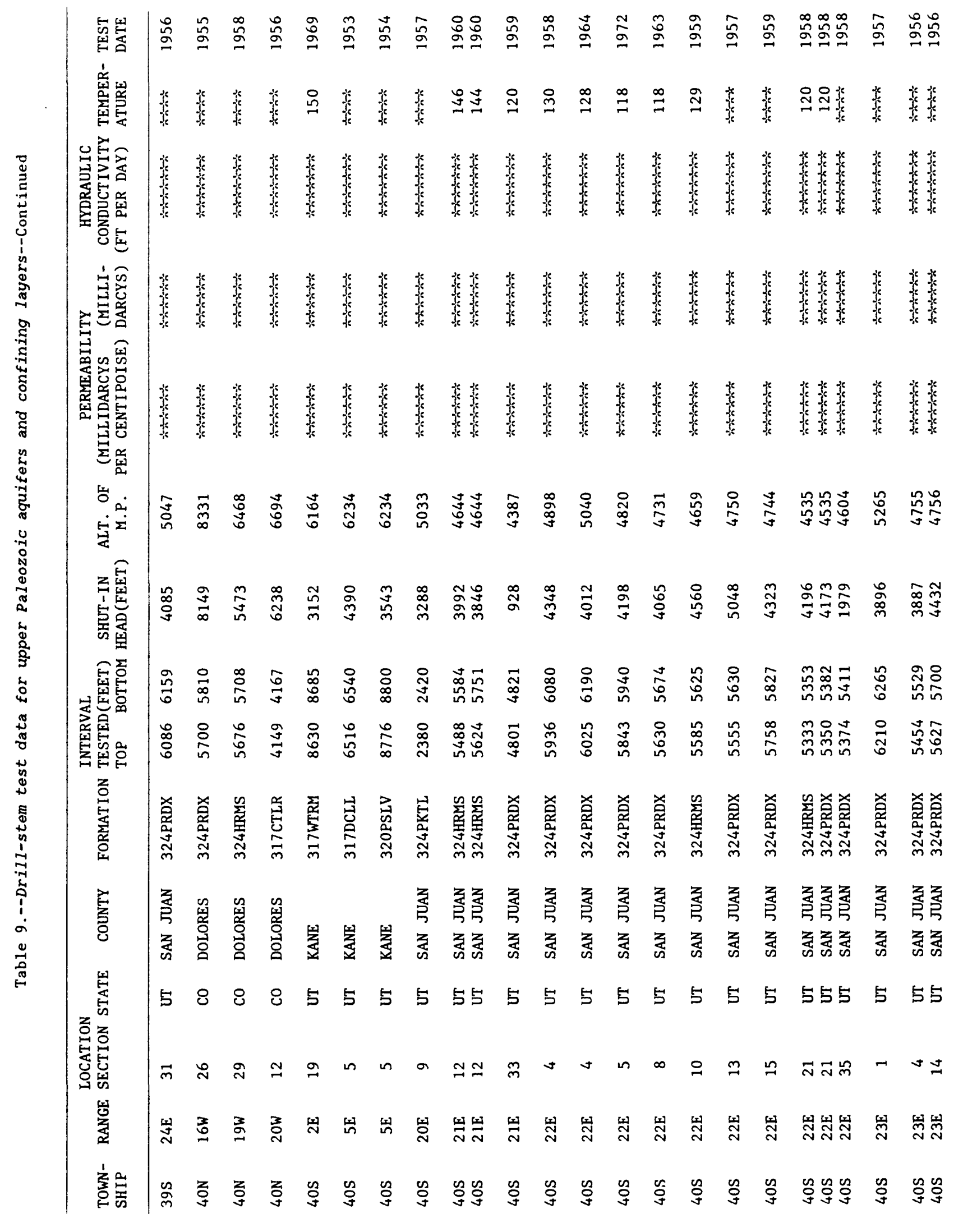




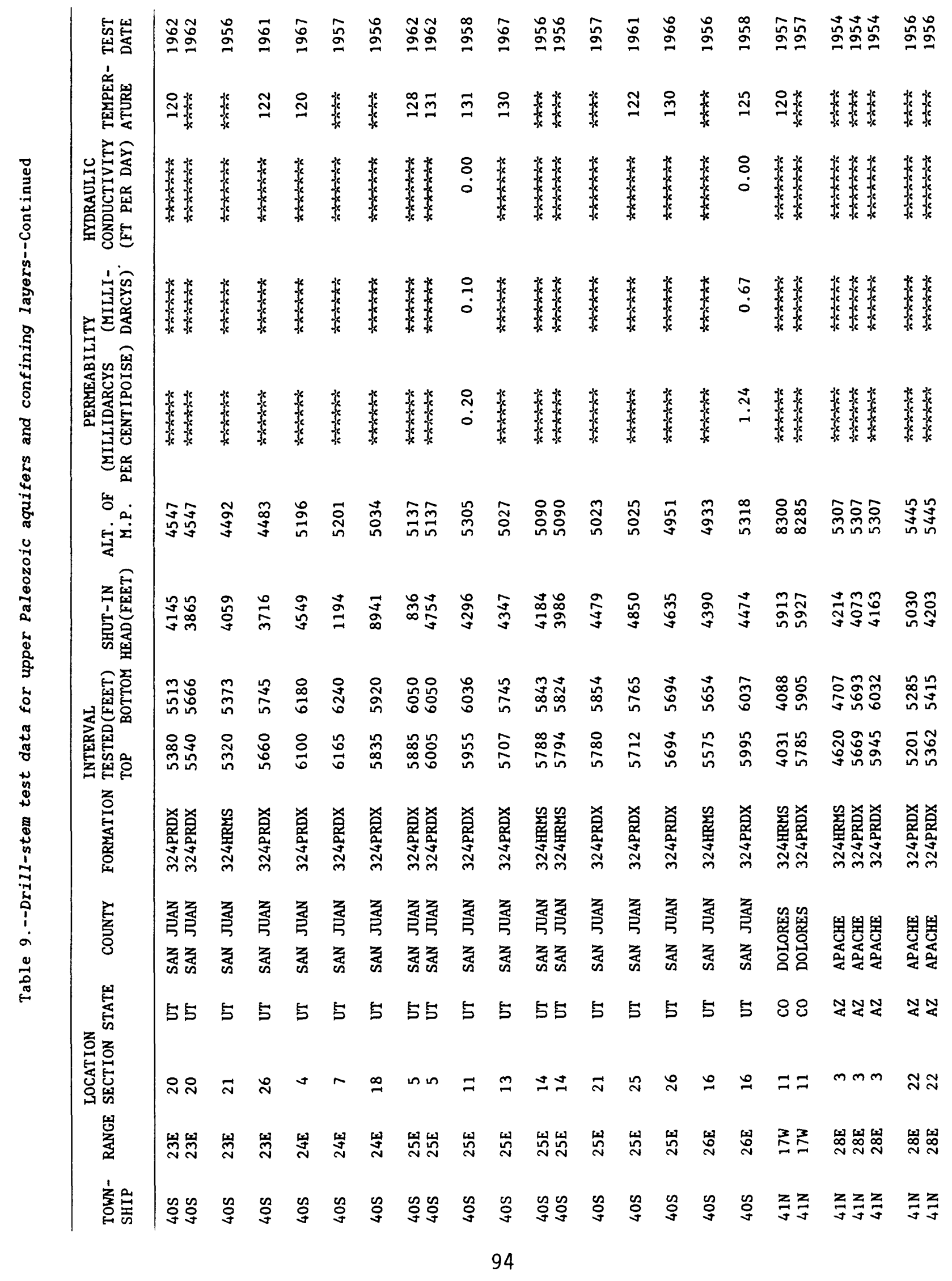




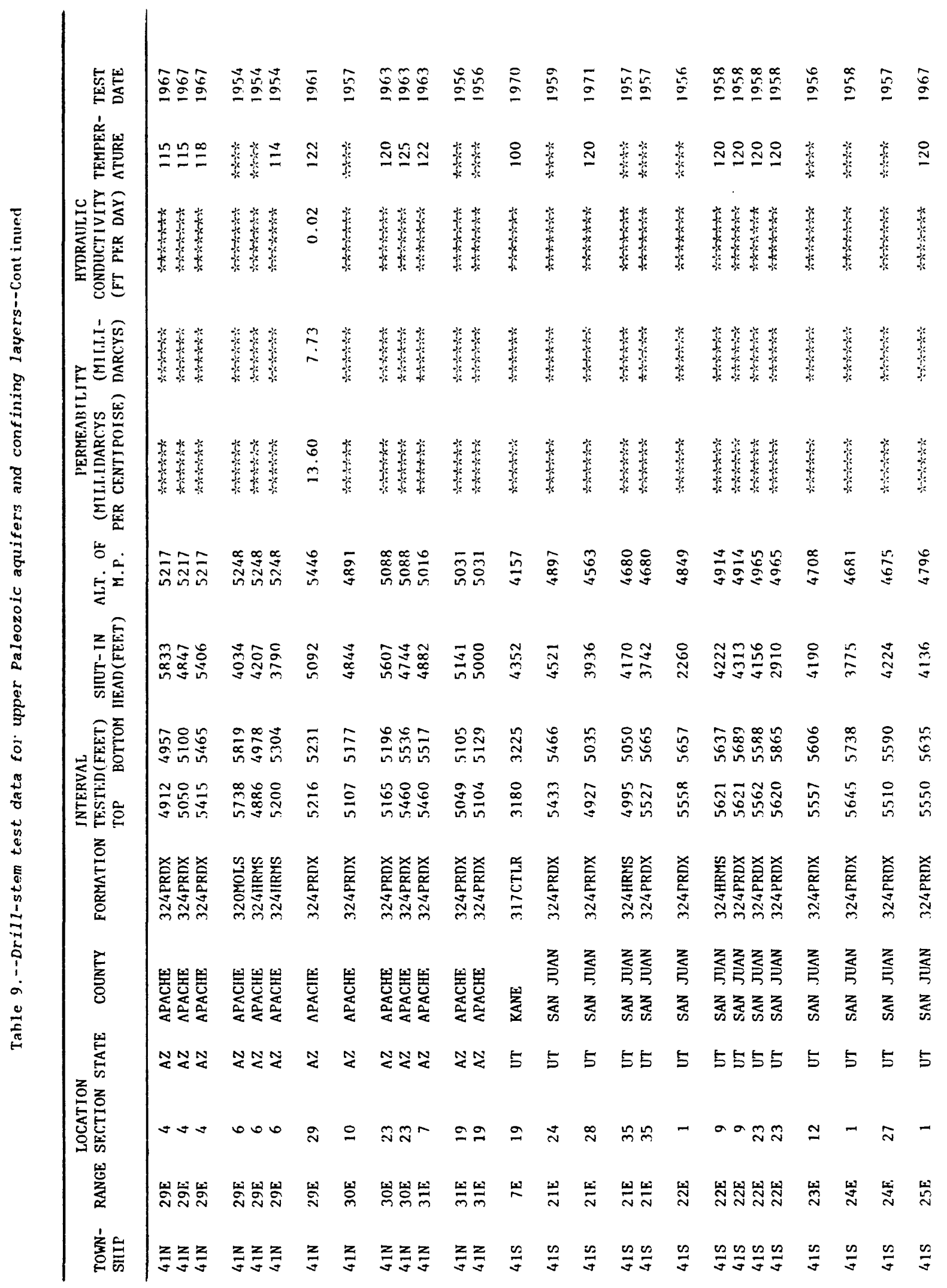




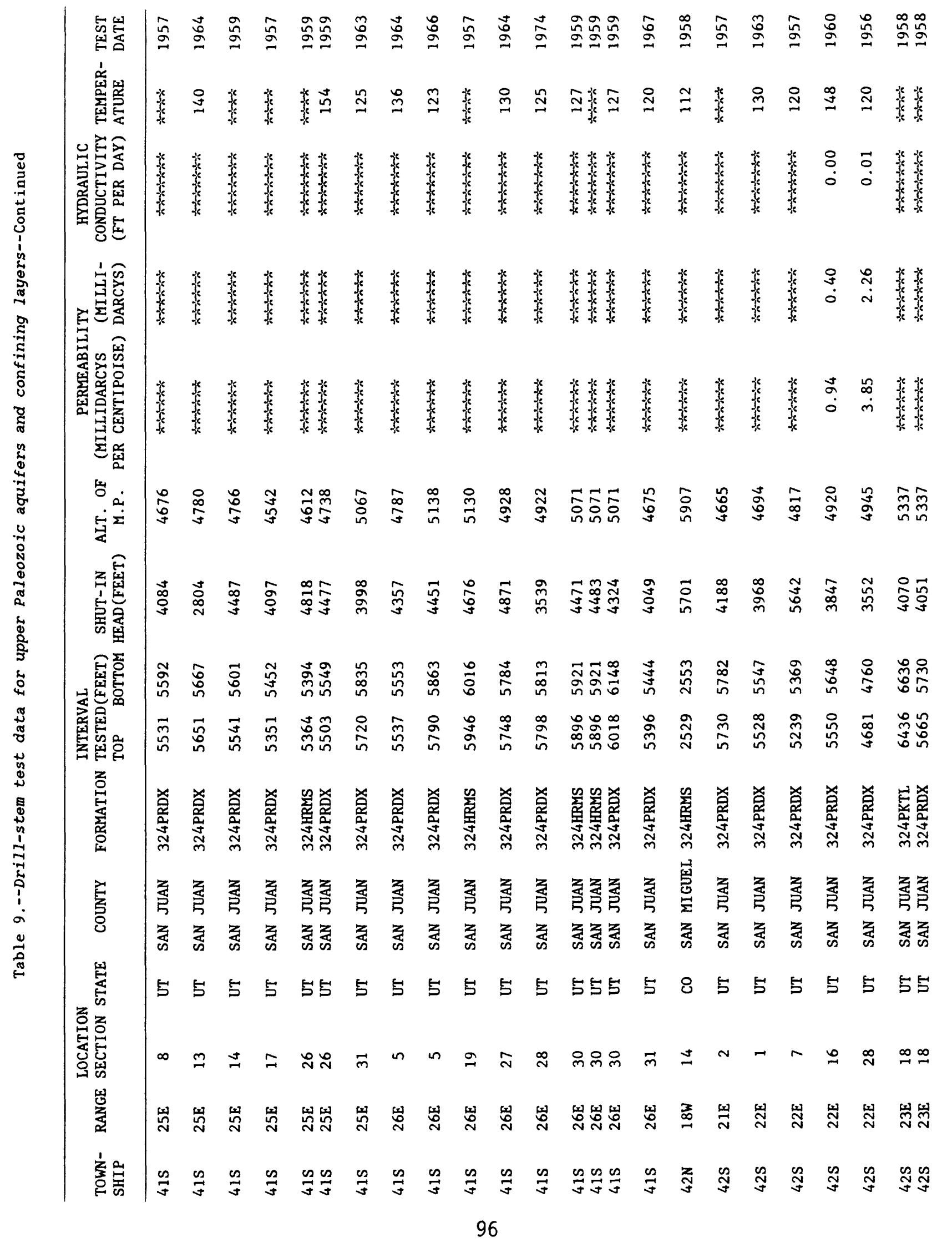




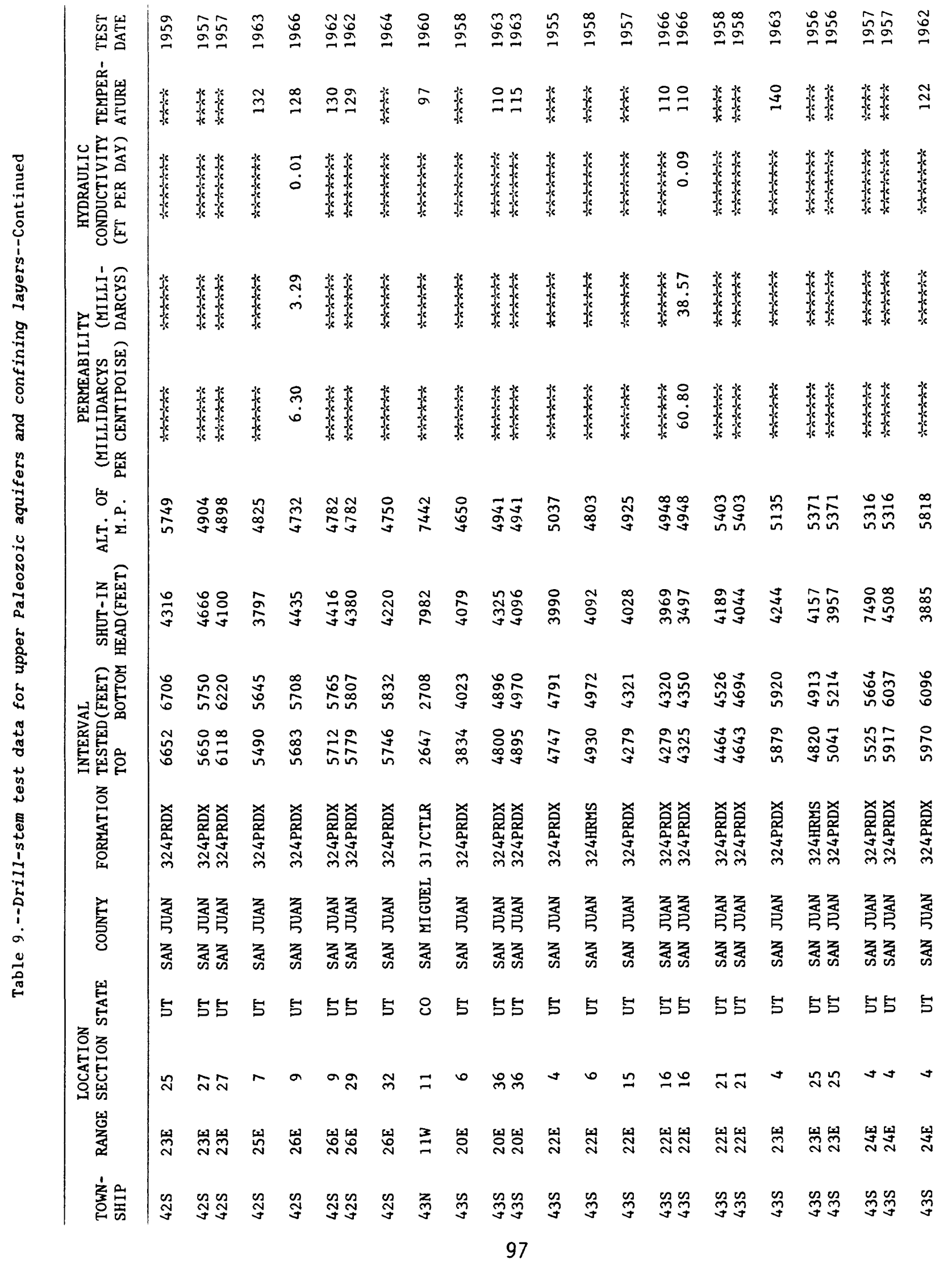




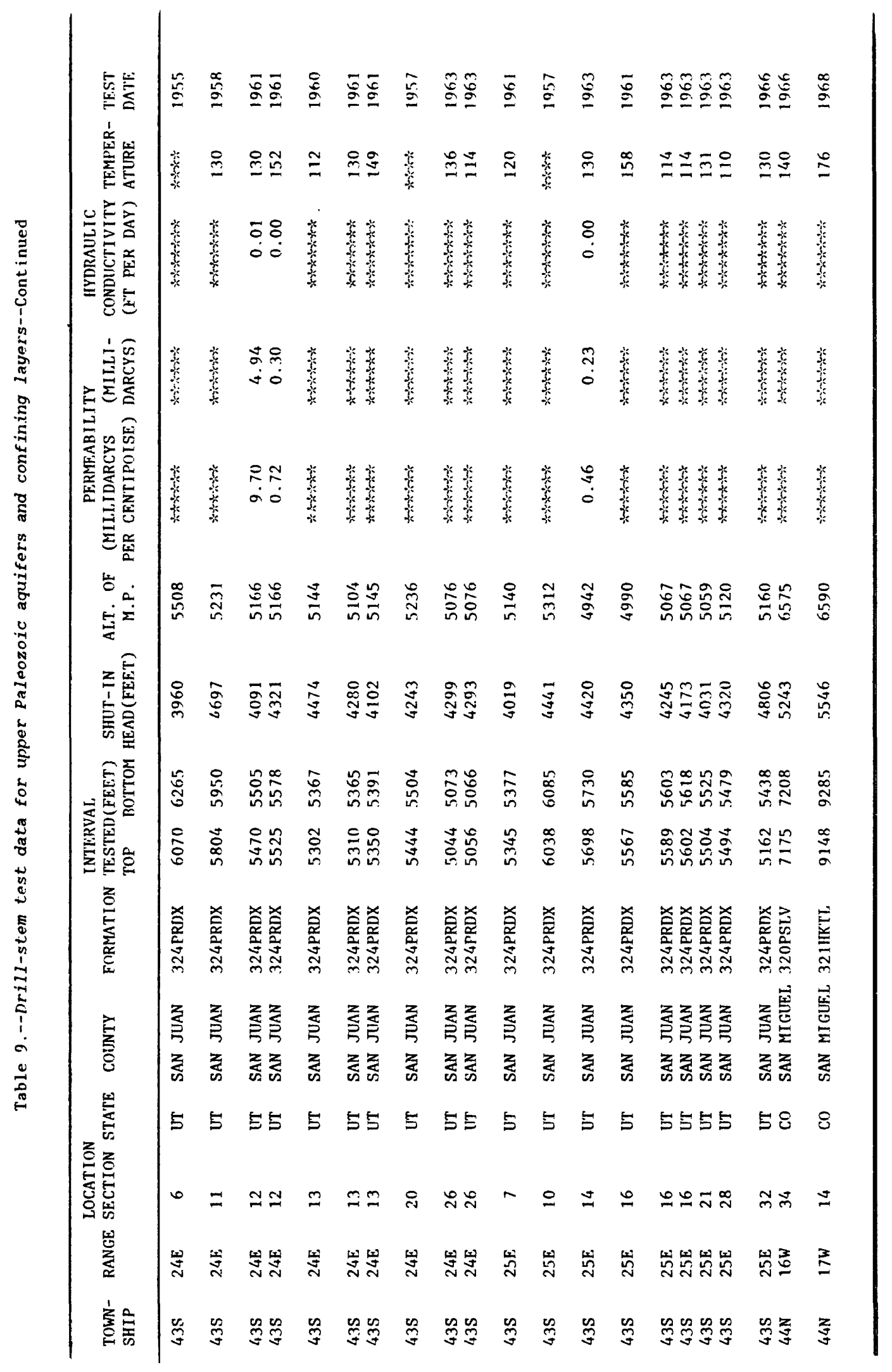




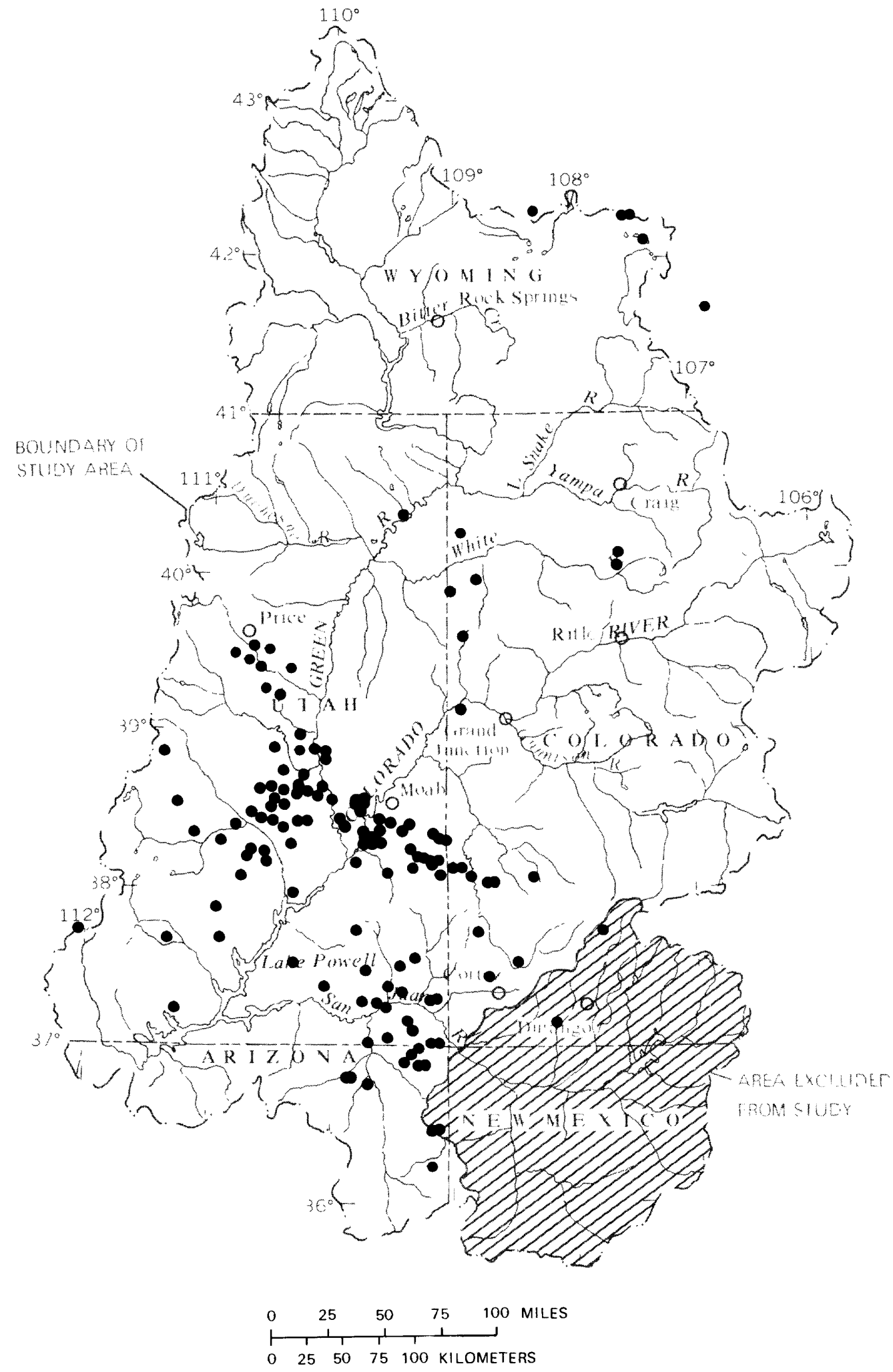

Figure 14.--Location of drill-stem test data for middle Paleozoic aquifers. 


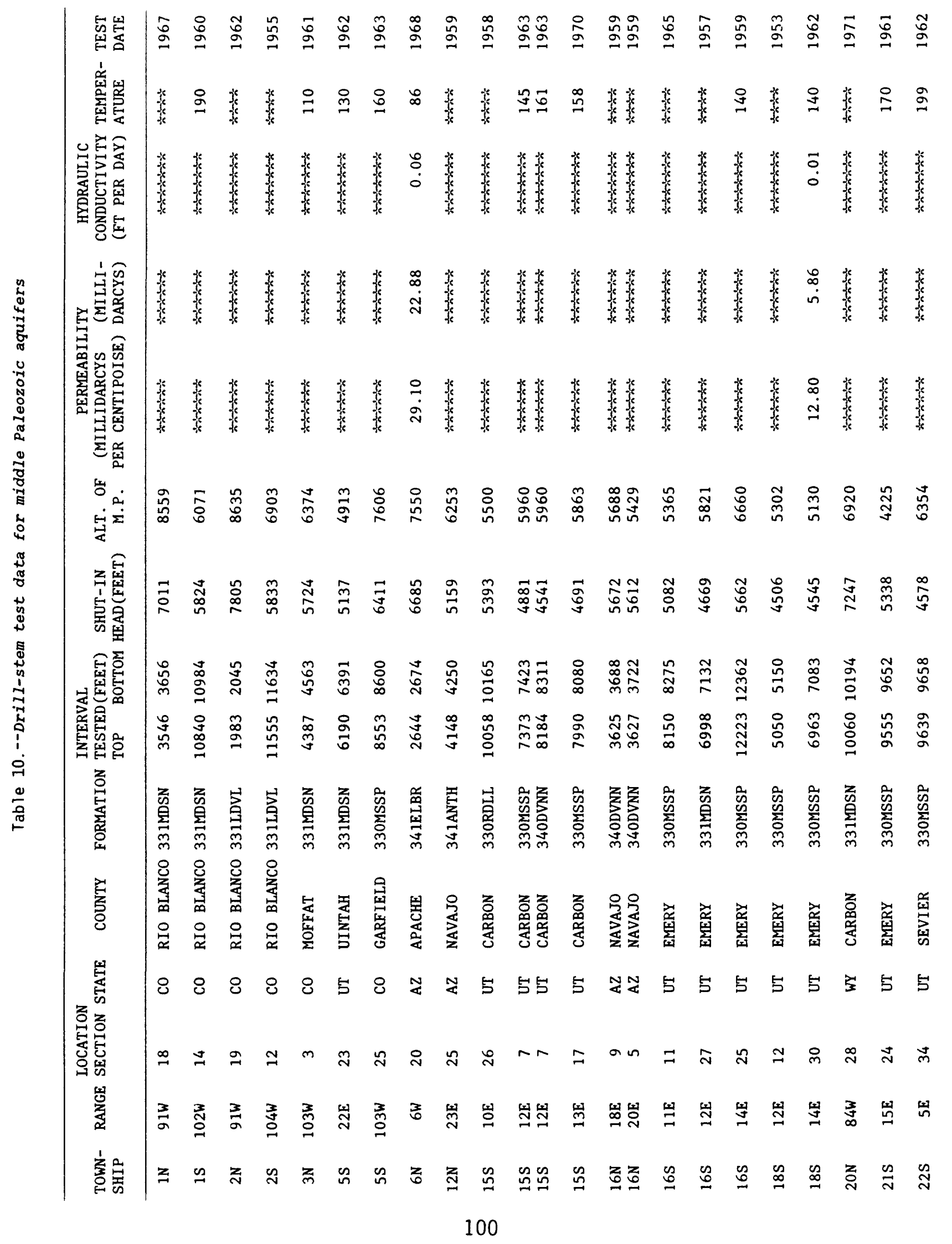




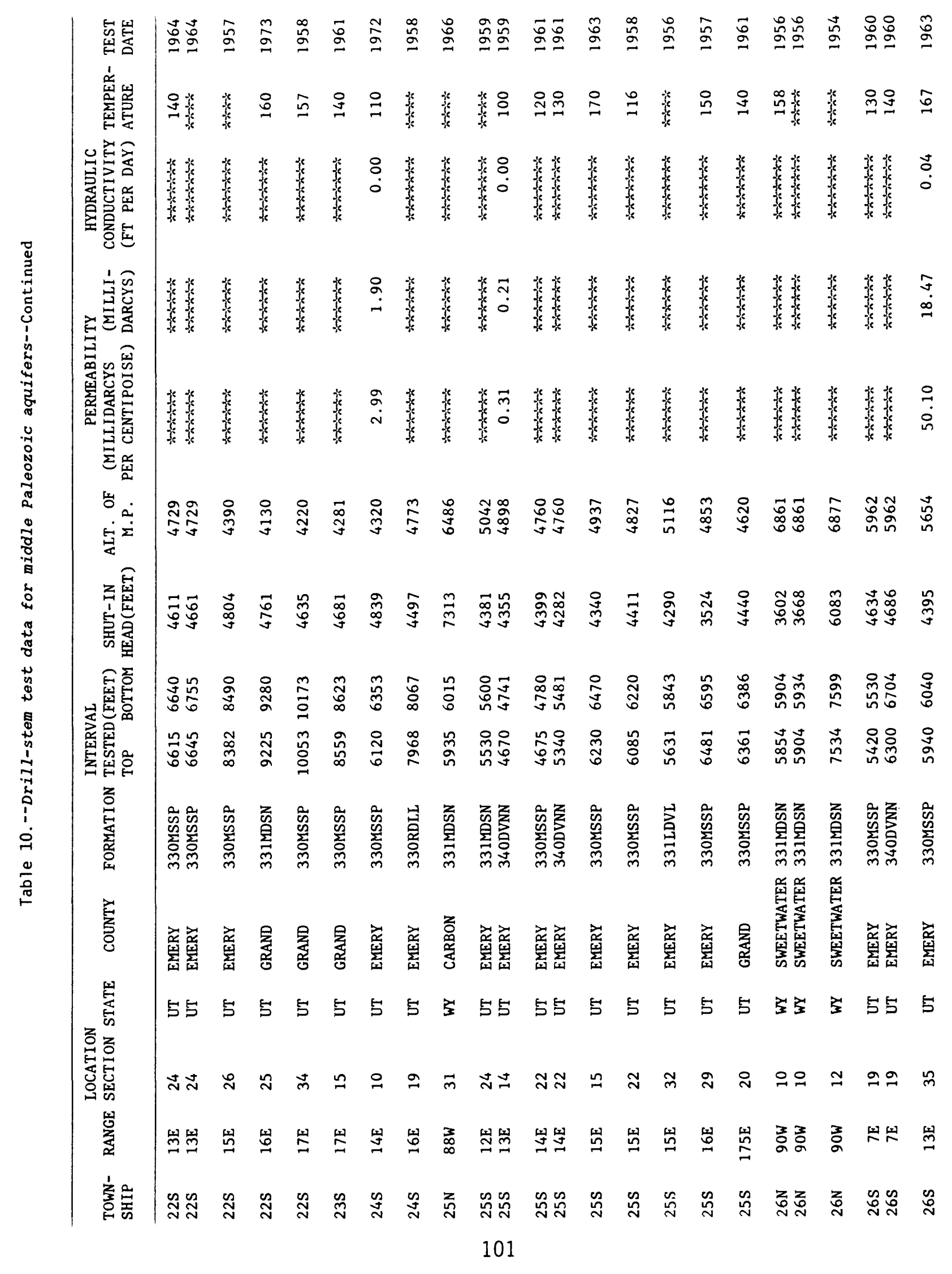




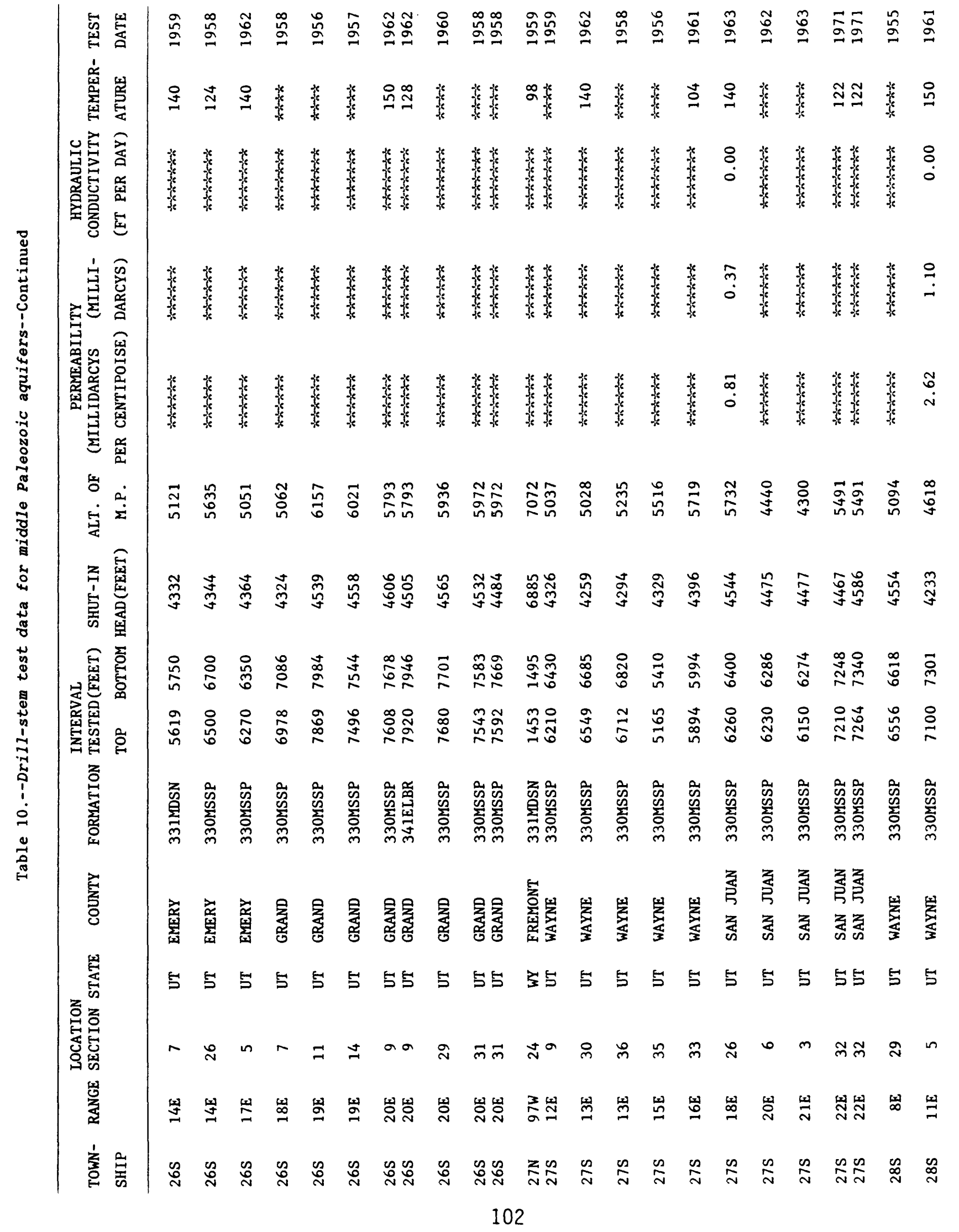




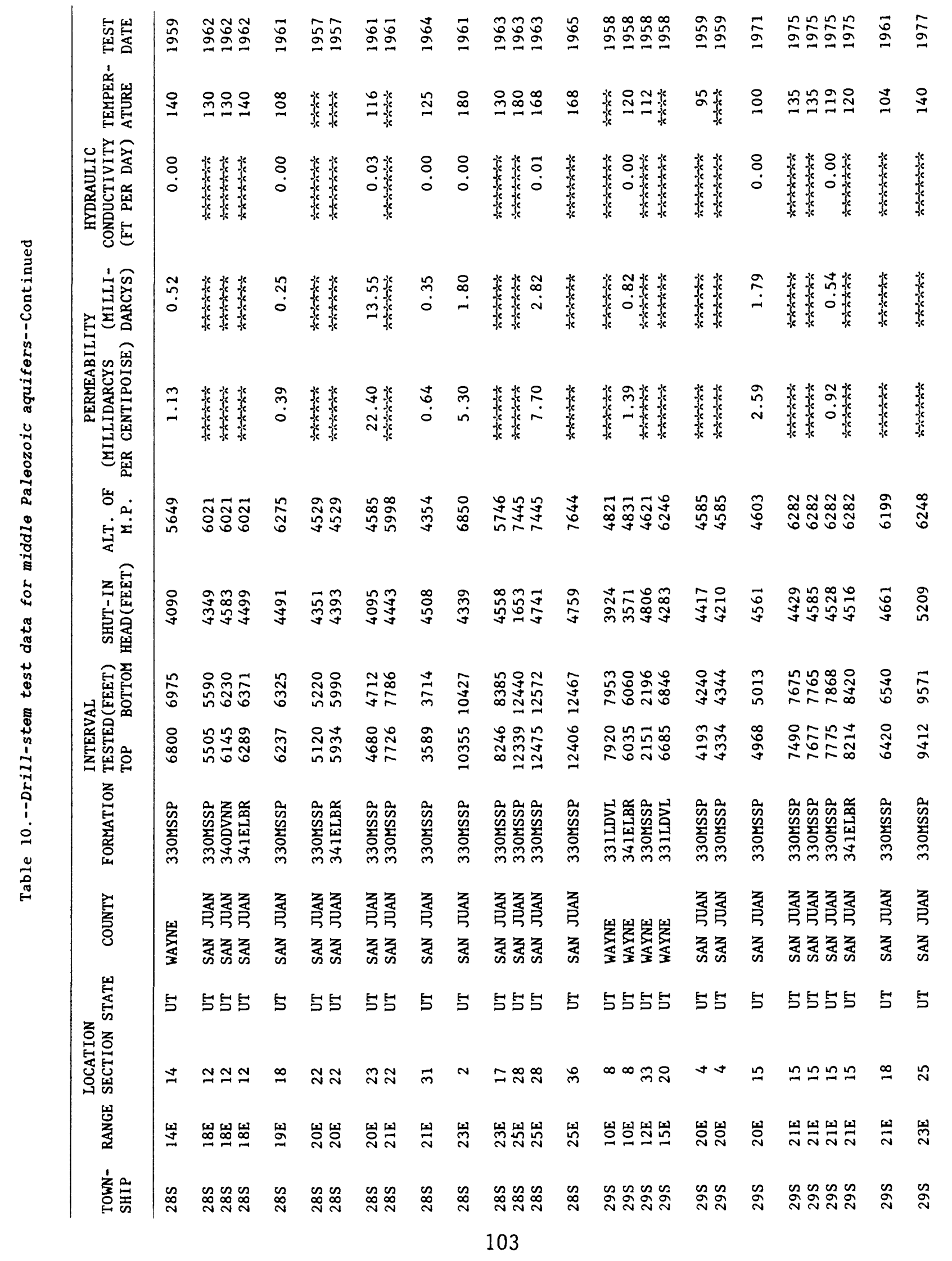




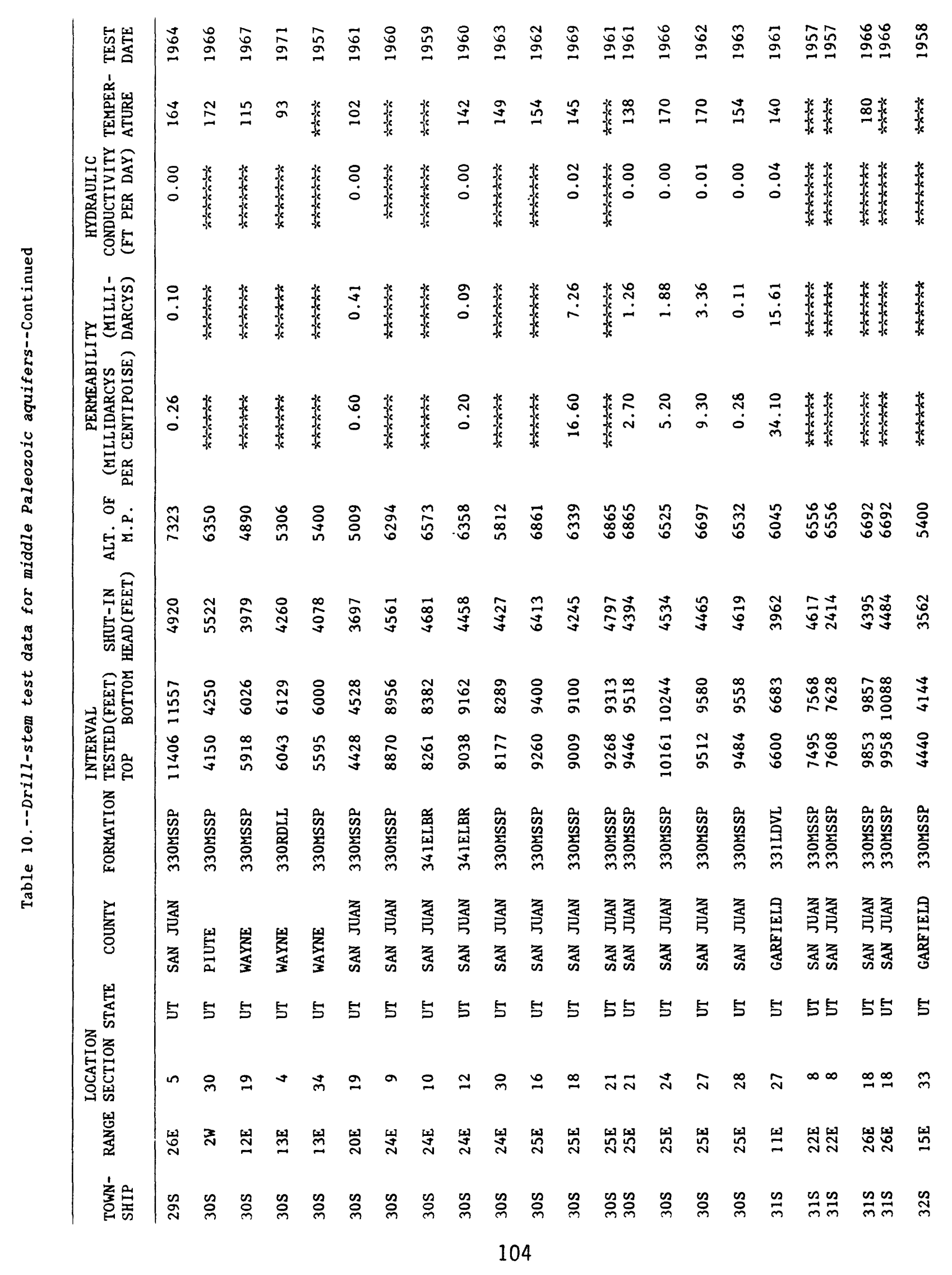




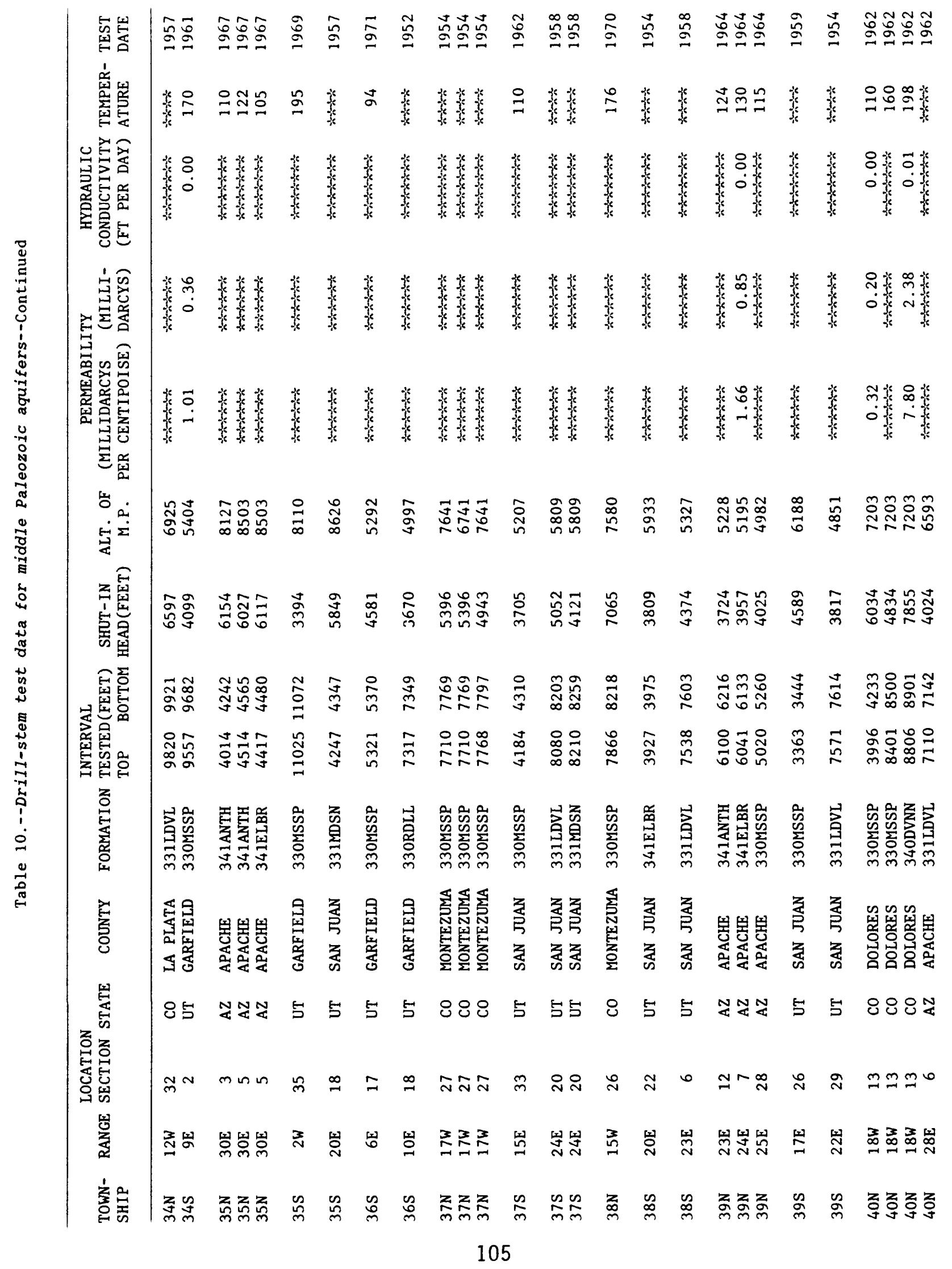




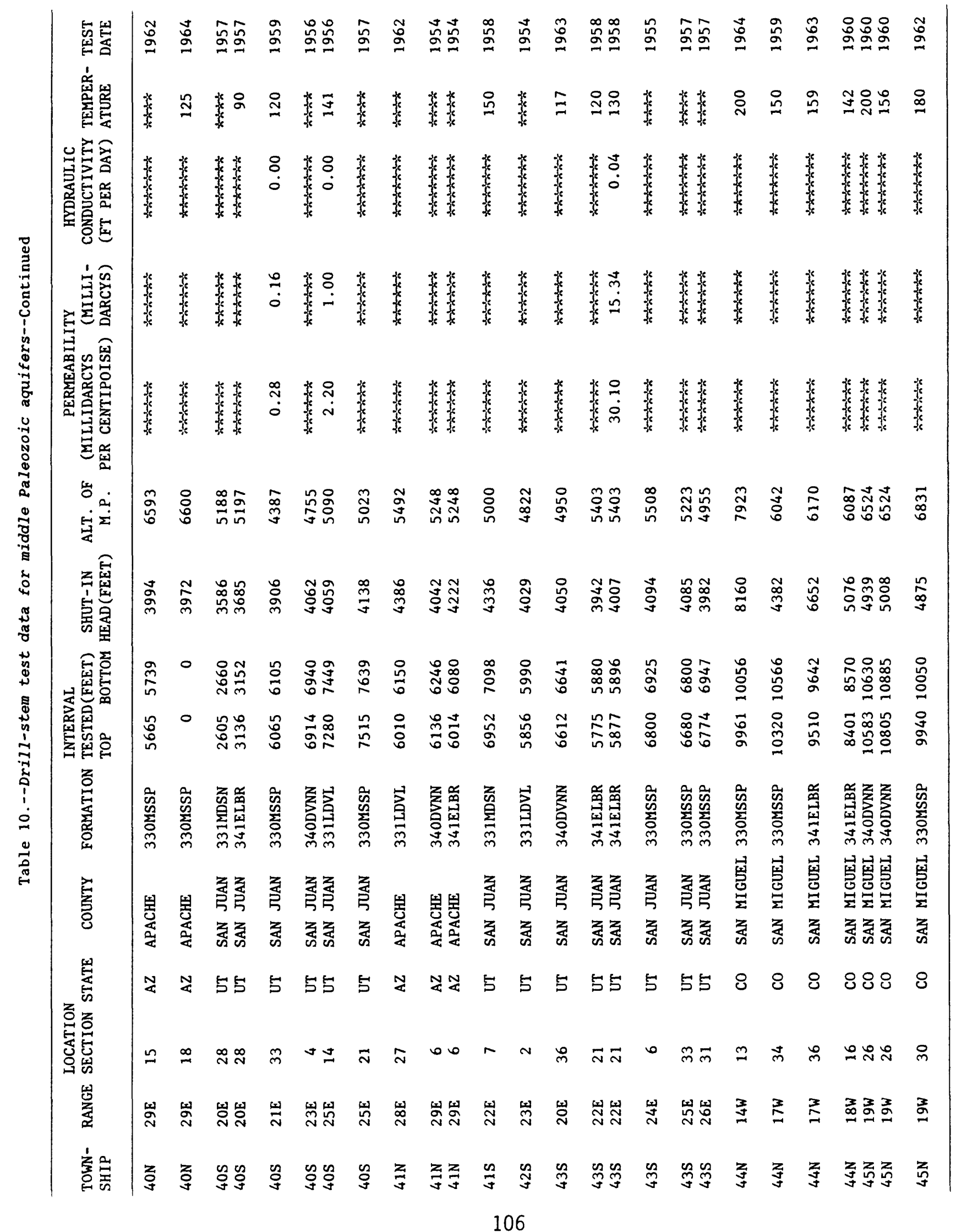




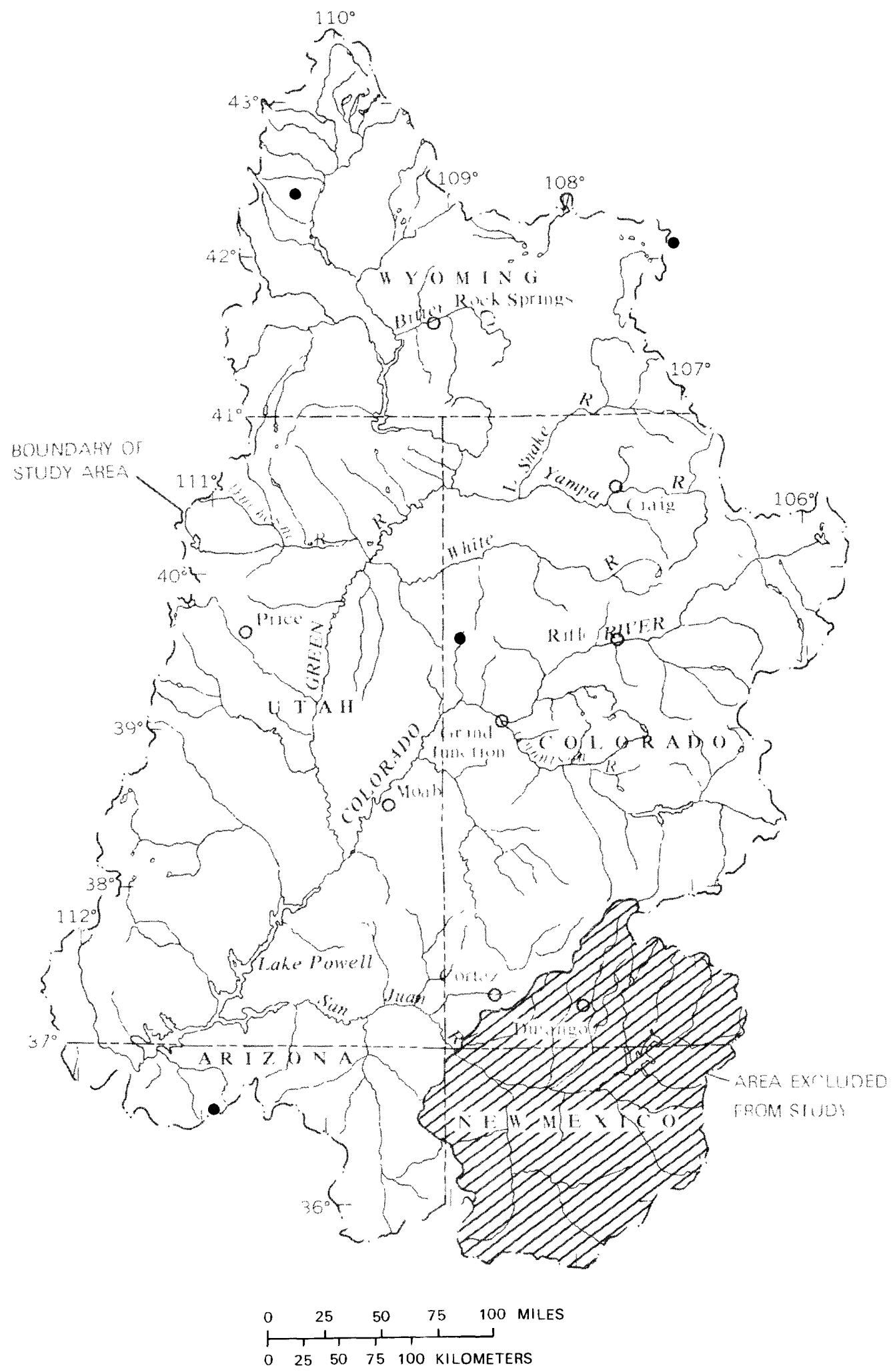

Figure 15.--Location of drili-stem test data for lower Paleozoic confining layers and aquifers. 


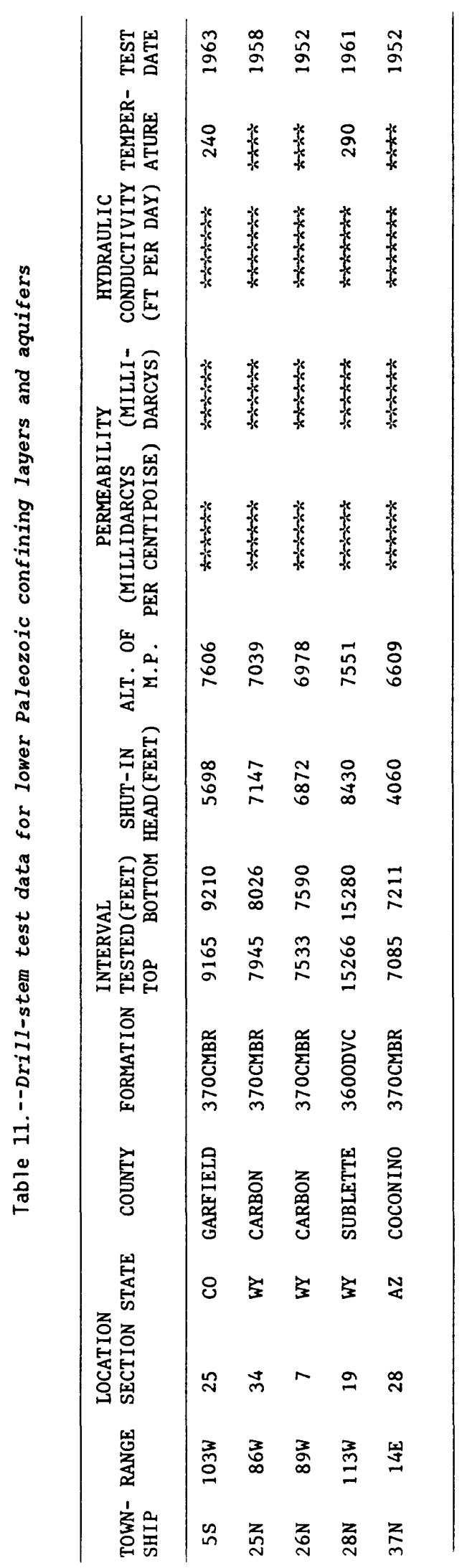




\section{SELECTED REFERENCES}

Baars, D. L., 1962, Permian System of Colorado Plateau: American Association of Petroleum Geologists Bulletin, v. 46, no. 2, p. 149-218.

Bowers, W. E., 1972, The Canaan Peak, Pine Hollow, and Wasatch Formations in the Table Cliff region, Garfield County, Utah: U.S. Geological Survey Bulletin 1331-B, $39 \mathrm{p}$.

Bradley, W. H., 1964, Geology of Green River Formation and associated Eocene rocks in southwestern Wyoming and adjacent parts of Colorado and Utah: U.S. Geological Survey Professional Paper 496-A, 86 p.

Bredehoeft, J. D., 1965, The dri11-stem test--The petroleum industry's deep-well pumping test: Groundwater, v. 3, no. 3, p. 31-36.

Cashion, W. B., 1967, Geology and fuel resources of the Green River Formation southeastern Uinta basin, Utah and Colorado: U.S. Geological Survey Professional Paper 548, 48 p.

1973, Geologic and structure map of the Grand Junction quadrangle, Colorado and Utah: U.S. Geological Survey Miscellaneous Investigations Series Map I-736, Scale 1:250,000.

Craig, L. C., 1981, Lower Cretaceous rocks, southwestern Colorado and southeastern Utah, in Geology of Paradox basin: Denver, Rocky Mountain Association of Geologists, 32nd Field Conference, Guidebook, p. 195-200.

Denson, N. M., 1965, Miocene and Pliocene rocks of central Wyoming, in Changes in stratigraphic nomenclature: U.S. Geological Survey Bulletin 1224-A, p. A70-77.

Denson, N. M., and Pipiringos, G. N., 1974, Geologic map and sections showing areal distribution of Tertiary rocks near the southeastern terminus of the Wind River Range, Fremont and Sweetwater Counties, Wyoming: U.S. Geological Survey Miscellaneous Investigations Map I-835, 2 sheets.

Denson, N. M., Zeller, H. D., and Stephenson, E. V., 1965, South Pass Formation on the southwest flank of Wind River Mountains, Wyoming, in Changes in stratigraphic nomenclature: U.S. Geological Survey Bulletin 1224-A, p. A27-29.

Fouch, T. D., 1976, Revision of the lower part of the Tertiary system in the central and western Uinta basin: U.S. Geological Survey Bulletin 1405-C, $7 \mathrm{p}$.

Fouch, T. D., Lawton, T. F., Nichols, D. J., Cashion, W. B., and Cobban, W. A., 1982, Chart showing preliminary correlation of major Albian to middle Eocene rock units from the Sanpete Valley in central Utah to the Book Cliffs in eastern Utah, in Nielson, D. L. ed., Overthrust belt of Utah: Utah Geological Association Publication 10, p. 267-272.

Grose, L. T., 1972, Tectonics, in Geologic atlas of the Rocky Mountain region: Denver, Rocky Mountain Association of Geologists, p. 35-44.

Hackman, R. J., and Wyant, D. G., 1973, Geology, structure, and uranium deposits of the Escalante quadrangle, Utah and Arizona: U.S. Geological Survey Miscellaneous Investigations Map I-744, 2 sheets, Scale 1:250,000.

Handbook of chemistry and physics, 1951, 33d ed.: Cleveland, Chemical Rubber Publishing Co., p. 1830-1831.

Hansen, W. R., 1969, The geologic story of the Uinta Mountains: U.S. Geological Survey Bulletin 1291, $144 \mathrm{p}$. 
Haynes, D. D., and Hackman, R. J., 1978, Geology, structure, and uranium deposits of the Marble Canyon $1^{\circ} \times 2^{\circ}$ quadrangle, Arizona: U.S. Geological Survey Miscellaneous Investigations Series Map I-1003, Scale $1: 250,000$.

Haynes, D. D., Vogel, J. D., and Wyant, D. G., 1972, Geology, structure, and uranium deposits of the Cortez quadrangle, Colorado and Utah: U.S. Geological Survey Miscellaneous Investigations Series Map I-629, Scale $1: 250,000$.

Hintze, Lehi, 1973, Geologic history of Utah: Provo, Utah, Brigham Young University Geology Studies v. 20, pt. 3, $181 \mathrm{p}$.

Horner, D. R., 1952, Pressure buildup in wells, in Proceedings of World Petroleum Congress (3rd), The Hague, Netherlands, 1951: Sec. II, p. 503-521.

Johnson, R. C., and May, Fred, 1980, A study of the Cretaceous-Tertiary unconformity in the Piceance Creek basin, Colorado: U.S. Geological Survey Bulletin 1482-B, 27 p.

Kent, H. C., and Porter, K. W., eds., 1980, Colorado geology: Denver, Rocky Mountain Association of Geologists, $258 \mathrm{p}$.

Knight, R. L., and Cooper, J. C., 1955, Suggested changes in Devonian terminology of the Four Corners area, in Geology of parts of Paradox, Black Mesa, and San Juan basins: Four Corners Geological Society 5 th Field Conference, Guidebook, p. 56-58.

Lines, G. C., and Glass, W. R., 1975, Water resources of the Thrust Belt of western Wyoming: U.S. Geological Survey Hydrologic Investigations Atlas HA-539, 3 sheets, Scale 1:250,000.

Lohman, S. W., 1972, Ground-water hydraulics: U.S. Geological Survey Professional Paper 708, p. 3-6.

Love, J. D., 1970, Cenozoic geology of the Granite Mountains area, centra] Wyoming: U.S. Geological Survey Professional Paper 495-C, 154 p.

Love, J. D., and Christiansen, A. C., 1980, Preliminary correlation of stratigraphic units used on $1^{\circ} \times 2^{\circ}$ geologic quadrangle maps of Wyoming, in Stratigraphy of Wyoming: Casper, Wyo., Wyoming Geological Association, p. 279-282.

Love, J. D., Christiansen, A. C., Bown, T. M., and Earle, J. L., compilers, 1979, Preliminary geologic map of the Thermopolis $1^{\circ} \times 2^{\circ}$ quadrangle, central Wyoming: U.S. Geological Survey Open-File Report 79-962, Scale 1: 250,000 .

MacLachlan, M. E., 1981, Stratigraphic correlation chart for western Colorado and northwestern New Mexico: Socorro, New Mexico Geological Society 32nd Field Conference, Guidebook, p. 75-79.

1984, General geology of the Piceance basin, in Taylor, 0. J., compiler, U.S. Geological Survey Professional Paper 1310 [in press].

Matthews, C. S., Russe11, D. G., 1967, Pressure buildup and flow tests in wells: New York, Society of Petroleum Engineers of AIME, $167 \mathrm{p}$.

Molenaar, C. M., 1981, Mesozoic stratigraphy of the Paradox basin--An overview, in Geology of the Paradox basin: Denver, Rocky Mountain Association of Geologists, 32nd Field Conference, Guidebook, p. 119-127. 
Molenaar, C. M., and Halvorsen, D. U., 1969, Nomenclature chart of the Grand Canyon and adjacent areas, in Geology and Natural History of the Grand Canyon Region: Four Corners Geological Society, 5th Field Conference, Guidebook, p. 68.

Mutschler, F. E., 1969, Desolation and Gray Canyons: Denver, Powell Society, $85 \mathrm{p}$.

Nelson, M. E., 1973, Age and stratigraphic relations of the Fowkes Formation, Eocene, of southwestern Wyoming and northeastern Utah: Laramie, University of Wyoming, Contributions to Geology, v. 12, no. 1, p. 27-31.

Oriel, S. S., and Platt, L. B., 1980, Geologic map of the Preston $1^{\circ} \times 2^{\circ}$ quadrangle, southeastern Idaho and western Wyoming: U.S. Geological Survey Miscellaneous Investigations Series Map I-1127, Scale 1:250,000.

Oriel, S. S., and Tracey, J. I., Jr., 1970, Uppermost Cretaceous and Tertiary stratigraphy of Fossil basin, southwestern Wyoming: U.S. Geological Survey Professional Paper 635, $53 \mathrm{p}$.

O'Sullivan, R. B., and Beikman, H. M., 1963, Geology, structure, and uranium deposits of the Shiprock quadrangle, New Mexico and Arizona: U.S. Geological Survey Miscellaneous Investigations Series Map I-345, Scale $1: 250,000$.

Peterson, Fred, and Pipiringos, G. N., 1979, Stratigraphic relations of the Navajo Sandstone to Middle Jurassic Formations, southern Utah and northern Arizona: U.S. Geological Survey Professional Paper 1035-B, 34 p.

Pipiringos, G. N., and Denson, N. M., 1970, The Battle Spring Formation in south-central Wyoming: Casper, Wyoming Geological Association, 22nd Annual Field Conference, Guidebook, p. 161-168.

Pipiringos, G. N., and O'Sullivan, R. B., 1978, Principal unconformities in Triassic and Jurassic rocks, western interior United States, a preliminary survey: U.S. Geological Survey Professional Paper 1035-A, $29 \mathrm{p}$.

Reynolds, M. W., 1976, Influence of recurrent Laramide structural growth on sedimentation and petroleum accumulation, Lost Soldier area, Wyoming: American Association of Petroleum Geologists Bulletin, v. 60, no. 1, p. 12-33.

Rocky Mountain Association of Geologists, 1972, Geologic Atlas of the Rocky Mountain region: Denver, $331 \mathrm{p}$.

Roehler, H. W., 1973, Stratigraphy of the Washakie Formation in the Washakie basin, Wyoming: U.S. Geological Survey Bulletin 1369, $40 \mathrm{p}$. 1974, Depositional environments of rocks in the Piceance Creek basin, Colorado, in Energy resources of the Piceance Creek basin, Colorado: Denver, Rocky Mountain Association of Geologists, 25th Field Conference, Guidebook, p. 57-64.

Rowley, P. D., Anderson, J. J., and Williams, P. L., 1975, A summary of Tertiary volcanic stratigraphy of the Southwestern High Plateaus and adjacent Great Basin, Utah: U.S. Geological Survey Bulletin 1405-B, $20 \mathrm{p}$.

Rowley, P. D., Tweto, Ogden, Hansen, W. R., and Carrara, P. E., 1979, Geologic map of the Vernal $1^{\circ} \times 2^{\circ}$ quadrangle, Colorado, Utah, and Wyoming: U.S. Geological Survey Miscellaneous Field Studies Map, MF-1163, Scale $1: 250,000$. 
Rubey, W. W., 1973, New Cretaceous Formation in the western Wyoming Thrust Be1t: U.S. Geological Survey Bulletin 1372-I, 35 p.

Rubey, W. W., Oriel, S. S., and Tracey, J. I., Jr., 1975, Geology of the Sage and Kemmerer 15-minute quadrangle, Lincoln County, Wyoming: $U$. S. Geological Survey Professional Paper 855, $118 \mathrm{p}$.

Shawe, D. R., Simmons, G. C., and Archbold, N. L., 1968, Stratigraphy of Slick Rock district and vicinity, San Miguel and Dolores Counties, Colorado: U.S. Geological Survey Professional Paper 576-A, 108 p.

Stewart, J. H., Poole, F. G., and Wilson, R. F., 1972, Stratigraphy and origin of the Chinle Formation and related upper Triassic strata in the Colorado Plateau region: U.S. Geological Survey Professional Paper 690, $336 \mathrm{p}$.

Swanson, R. W., Hubert, M. L., Luttre11, G. W., and Jassen, V. M., 1981, Geologic names of the United States through 1975: U.S. Geological Survey Bulletin 1535, $643 \mathrm{p}$.

Tweto, Ogden, 1976, Geologic map of the Craig $1^{\circ} \times 2^{\circ}$ quadrangle, northwestern Colorado: U.S. Geological Survey Miscellaneous Investigations Series Map I-972, Scale 1:250,000.

Upper Colorado Region State-Federal Inter-Agency Group, 1971, Upper Colorado region comprehensive framework study, Appendix I and Appendix II: p. II-1-15.

Weiss, Emanuel, 1982, A computer program for calculating relativetransmissivity input arrays to aid model calibration: U.S. Geological Survey Open-File Report 82-447, $18 \mathrm{p}$.

Welder, G. E., and McGreevy, L. J., 1966, Ground-water reconnaissance of the Great Divide and Washakie basins and some adjacent areas, southwestern Wyoming: U.S. Geological Survey Hydrologic Investigation Atlas HA-219, 3 sheets, Scale 1:250,000.

Wengerd, S. A., and Strickland, J. W., 1954, Pennsylvanian stratigraphy of Paradox Salt basin, Four Corners region, Colorado and Utah: American Association of Petroleum Geologists Bulletin, v. 38, no. 10, p. 2157-2199; Correction, v. 39, no. 2, 1955, p. 259. 